\title{
Characterizing the activity of PPE proteins in the frizzled/PCP signaling pathway
}

\author{
Ying Wang \\ Beijing, China
}

B.S. Biotechnology, Beijing University of Technology, China, 2009

A Dissertation presented to the Graduate Faculty

of the University of Virginia in Candidacy for the Degree of

Doctor of Philosophy

Department of Biology

University of Virginia

May, 2016 


\begin{abstract}
The frizzled/starry night signaling pathway is a non-canonical Wnt signaling pathway which controls planar cell polarity in both vertebrates and invertebrates. Genetic studies using Drosophila melanogaster identified a set of genes which control planar cell polarity and those genes were placed into three phenotypic and epistasis group in the frizzled/starry night signaling pathway. This dissertation reports on the molecular and genetic studies of downstream components - the planar cell polarity effector genes, inturned (in), fuzzy (fy) and fritz (frtz) and their relationship with upstream planar cell polarity core genes, such as disheveled $(d s h)$.
\end{abstract}

In chapter 2, I describe the context dependent epistatic relationship between downstream planar cell polarity effector genes and upstream core genes. I have found that when over expressed the planar cell polarity effector proteins can alter both the subcellular location and level of accumulation of the upstream proteins. I also showed that In, Fy and Frtz form a protein complex within the wing cells and modulate the accumulation of each other. I further found that over expression of Frtz results in a marked delay in hair initiation suggesting that it has a separate role/activity in regulating the cytoskeleton that is not shared by other members of the group.

In Chapter 3, I describe a set of experiments to map the residues required for the interaction between In and Frtz WD40 fragment. Also, I found a direct interaction between Frtz or Fy with planar cell polarity core protein Dsh, suggesting the asymmetric accumulations of planar cell polarity effector proteins are mediated by physical interactions with Dsh. The interaction between Frtz and Dsh requires multiple fragments in the Frtz protein. Furthermore, I describe a newly generated in null mutant, in precise deletion $\left(i n^{P D}\right)$ using the CRISPR/Cas9 
genome editing technique and found that Frtz has both In independent and dependent functions in Drosophila wing cells as the mild overexpression of Frtz partially rescued the In loss of function mutant.

In Chapter 4, I describe a newly generated frtz $^{\text {mNeonGreen }}$ stock (made using the CRISPR/Cas9 genome editing technique) for studying the Frtz protein activity in Drosophila. I modified the frtz gene in the Drosophila genome by adding a green fluorescent protein mNeonGreen on the carboxyl terminus of frtz. This modified gene provides full rescue of $f r t z$ and is good for live imaging of the Frtz protein in various tissues, such as wing, thorax, abdomen and arista. Time-lapse imaging data shows Frtz protein is preferentially distributed to discrete membrane subdomains ("puncta"), and the puncta are very dynamic at the subcellular level. Fluorescence recovery after photo-bleaching shows Frtz protein in various tissues has a similar recovery rate. 


\section{Acknowledgements}

A highly collaborative effort gives birth to this dissertation, and I would like to thank many people for leading me through this process. I am most grateful to my advisor Dr. Paul Adler, whose support has been throughout the whole process. His encouragement and guidance has helped me solve many complicated issues in my research. I have been always inspired by having a conversation with him when I have questions about my research. I was also motivated by his passion for doing research.

I also would like to give my thanks to the members of my research committee, Dr. Raymond Keller, Dr. Ignacio Provencio, Dr. Adrian Halme and Dr. Zhen Yan. At each annual report meeting, they always give me many valuable suggestions. Meanwhile, I really appreciated all of the help from past and current labmates, Dr. Qiuheng Lu, DeSean Thom, Magdalena Brymerska, Lukasz Sobala, Waheeda Naimi and Nella Solodukhina. They have been making the lab a pleasurable and productive environment to work in. Among them, I am especially thankful to Dr. Qiuheng Lu, and DeSean Thom for teaching me in many bench works. The execution of my dissertation research also depended on many other people in biology department at UVa. There are too many to name them all, but I would like to specifically thank Dr. Ammasi Periasamy and Zdenek Svindrych at Keck Center for Cellular Imaging. Without their help for imaging techniques, this dissertation wouldn't be finished.

During my PhD study, I felt lucky and was very grateful to the institution that rewarded me fellowship to support my research. For instance, I was fortunate enough to receive the dissertation year fellowship from my department. 
Finally, I would like to thank my family both here and in China. Their unconditional support and encouragement has helped me through my PhD program. My husband, Xiaozeng Yang, has been a constant source of love, support and motivation, and he is always being there, both in life and science, which I am deeply grateful. My parents, they have given me so much. They have left me with a debt that I can never repay but will remember forever. 


\section{Table of contents}

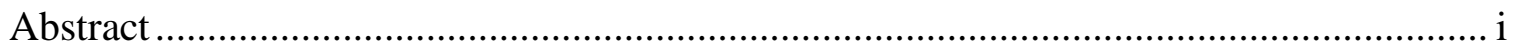

Acknowledgements ...................................................................................................ii

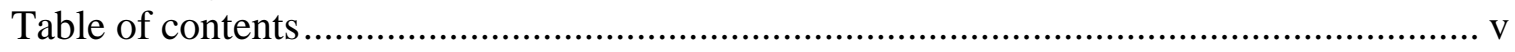

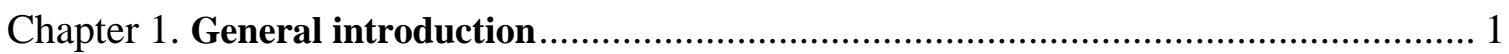

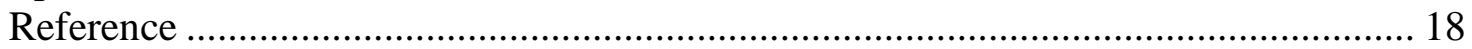

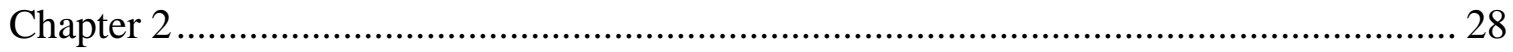

The proteins encoded by the Drosophila Planar Polarity Effector genes inturned, fuzzy and fritz interact physically and can re-pattern the accumulation of "upstream" Planar

Cell Polarity proteins

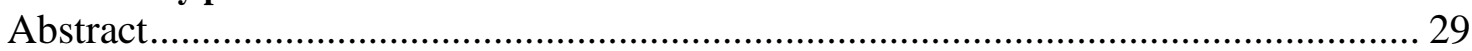

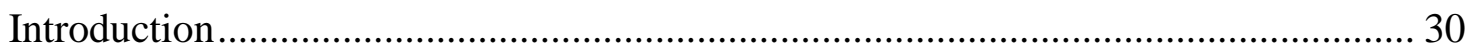

Materials and methods .................................................................................... 34

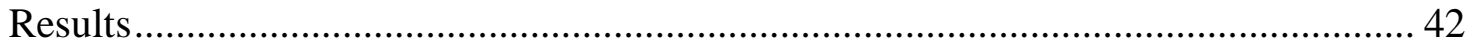

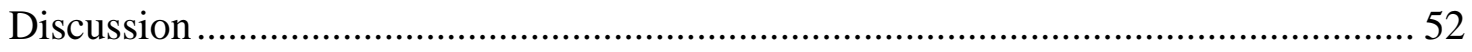

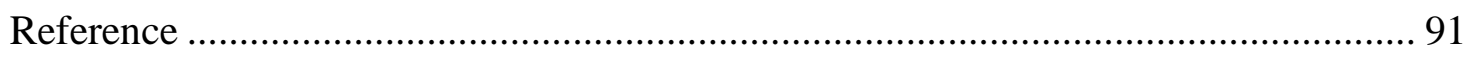

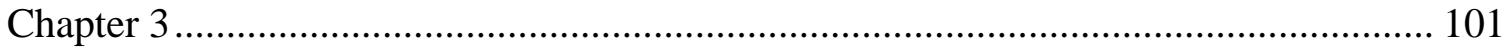

Planar cell polarity effector fritz has inturned independent function and physically interacts with upstream core gene disheveled

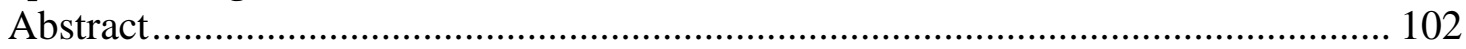

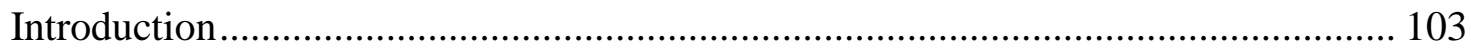

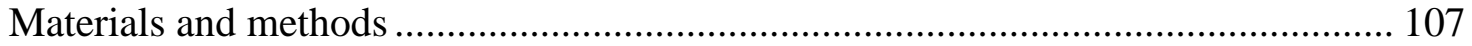

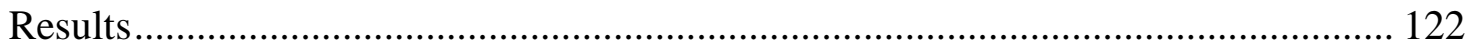

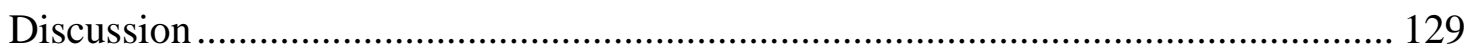

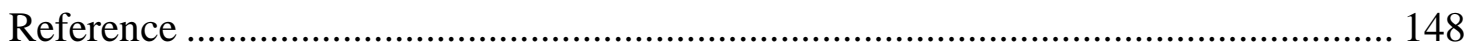

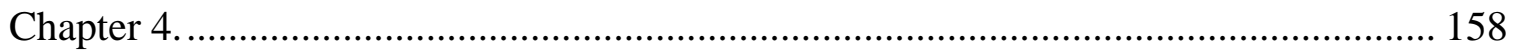

Dynamic property of planar cell polarity effector protein Fritz in various tissues of Drosophila

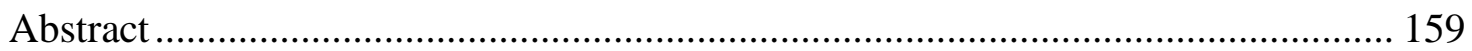

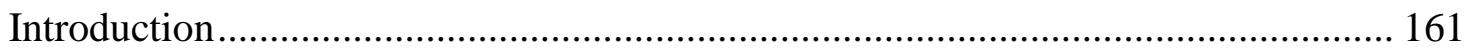

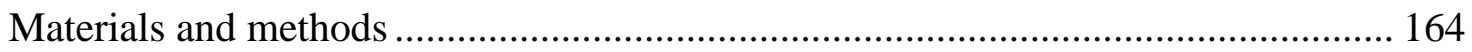

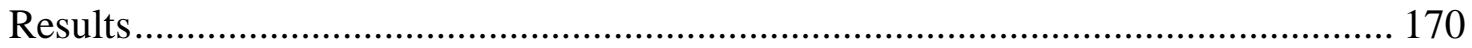

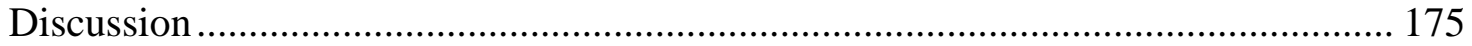

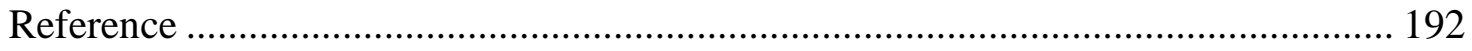

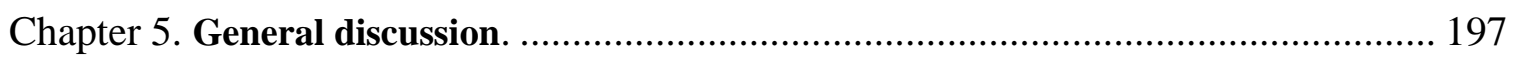

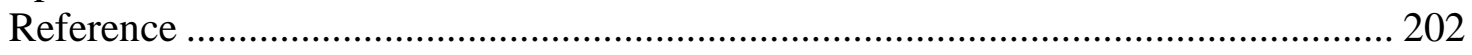




\section{Chapter 1}

\section{General Introduction}

\section{Planar cell polarity}

Epithelia, one of the four basic structures (nervous tissues, connective tissues, muscles and epithelia) of animal tissues line the internal cavity and surface of the body. Epithelial cells adhere to one another through adherens junctions and tight junctions, and need to maintain both the apical-basal polarity and planar cell polarity (PCP). All epithelia feature an apical-basal polarity which is described as polarity orthogonal to the plane of the tissue (Figure1). This structural feature allows organs and tissues to secrete and take up material in a polarized manner and allows the tissue to function as a barrier between internal and external space. In addition, most epithelial tissue is also polarized within the plane of the epithelium in which specialized structures are oriented within the plane of the epithelial sheet. Polarization of a field of cells within the plane of the cell sheet is defined as PCP (Figure 1).

The coordination of cellular polarity is an important feature of animal development, and it is critical for patterning, maintenance and homeostasis of individual organ, system and the organism as a whole. PCP has been found to be involved in a wide variety of animal developmental processes. The coordinate alignment of scales in fish, feathers in birds and hairs in mammals are easily visualized examples of PCP. However, PCP also regulates the development of internal organs and tissues. The stereocilia in mouse inner ear and ommatidia in Drosophila eye show PCP in their uniform orientation (Dabdoub and Kelley, 2005; Wolff and Rubin, 1998; Zheng et al., 1995). PCP also play a key role in polarized tissue movements during vertebrate gastrulation, in a process known as convergent extension (Keller et al., 2000; Wallingford et al., 2002; Wallingford et al., 2000). In Drosophila melanogaster, PCP regulates 
development of epidermal hairs (trichomes) (Wong and Adler, 1993), bristle sensory organs (Adler, 2002), embryonic denticles (Donoughe and DiNardo, 2011; Price et al., 2006; Repiso et al., 2010) and eye ommatidial clusters (Zheng et al., 1995), etc . Dysregulation of PCP has been connected to various human diseases, including but not limited to neural tube defects, polycystic kidney disease, congenital heart disease, etc. (Curtin et al., 2003; Fischer et al., 2006; Hamblet et al., 2002; Henderson et al., 2001; Henderson et al., 2006).

\section{PCP in Drosophila}

Various tissues of adult and larval Drosophila display PCP, and Drosophila has been the key model system for studying the genetic basis for PCP. The simplest form of PCP in Drosophila is seen in the alignment of epidermal hairs (trichomes) on the wing and abdomen (Figure 2A, 2A' and 2B')(Gubb and Garcia-Bellido, 1982; Wong and Adler, 1993). Drosophila wing is flat and is derived from two layers of epidermal cells. Each wing cell forms a relatively long distally pointing hair. The simplicity of this system made it the principal tissue for studying PCP in the fly. More complex examples of PCP in Drosophila are the alignment of sensory bristles (Figure 2B and 2B'), which depends on the proper orientation of asymmetric cell divisions (Fichelson and Gho, 2003; Gho et al., 1999), and the ommatidia of the compound eye (Figure 3B and 3C), in which cell fate specification and coordinated rotation of cell clusters are keys to forming appropriate polarity (Maung and Jenny, 2011).

\section{PCP in Drosophila Wing}

The adult cuticular wing is formed from the pupal wing which is comprised of two layers of primarily hexagonal epithelial cells (Wong and Adler, 1993). Each wing cell produces a single actin-rich hair or trichome at the distal vertex of the cell (Figure $2 \mathrm{~A}^{\prime} \& 3 \mathrm{~A}$ ). The frizzled $(f z) /$ starry night (stan) signaling pathway controls PCP in Drosophila wing by 
restricting the site of hair initiation to the distal most part of the cell (Figure 3A) (Wong and Adler, 1993).

PCP in the Drosophila sensory bristle

The cuticular surface of Drosophila is decorated with many sensory bristles. The sensory bristles are organized in a polarized fashion such that bristles on the thorax and abdomen point posteriorly and on appendages they point distally (Figure 2B \& B') (Adler, 2002; Adler, 2012). The sensory bristle polarity is controlled by $f z / s t a n$ pathway which orients the spindle for the first differentiative division of the sensory organ precursor cell during the determinative cell divisions (Gho and Schweisguth, 1998; Lu et al., 1999). PCP also controls the location of bract on Drosohila legs as it is routinely proximal to the bristle (Figure 2B') (Adler, 2002).

PCP in the Drosophila compound eye

The adult Drosophila eye contains about 800 ommatidia. (Singh et al., 2005);Wolff and Ready, 1993). PCP is evident in the orientation of the dorsal and ventral ommatidia, which are mirror images of each other (Figure 2C \& C') (Wolff and Ready, 1993). Ommatidial differentiation is marked by the progression from posterior to anterior of the morphogenetic furrow. Each ommatidium is comprised of 20 cells including 8 photoreceptor cells, R1-R8 (Figure 2C') (Adler, 2012). The $f z /$ stan pathway controls PCP in ommatidia by regulating the R3/R4 cell fate decision (Zheng et al., 1995). These cell fate decisions are determined by a PCP competition in which R3 cell having higher frizzled $(f z)$ activity and R4 maintaining higher Van gogh (vang) activity (Tomlinson and Struhl, 1999; Zheng et al., 1995).

\section{The frizzled ( $f$ z)/starry night (stan) signaling pathway}


A great deal of effort has been made to understand which genes play a role in regulating PCP and how PCP is established during animal development. The most informative of these have used Drosophila as model system (Adler, 2002; Donoughe and DiNardo, 2011; Price et al., 2006; Repiso et al., 2010; Wong and Adler, 1993). Over the past several decades, genetic studies in Drosophila have led to the identification and characterization of a number of genes which are essential for PCP. These genes comprise the $f z /$ stan signaling pathway (Adler et al., 1998; Chae et al., 1999; Collier and Gubb, 1997; Collier et al., 2005; Feiguin et al., 2001; Gubb and Garcia-Bellido, 1982; Klingensmith et al., 1994; Strutt and Warrington, 2008; Taylor et al., 1998; Yang et al., 2002; Zeidler et al., 1999). In recent years it has become clear that this conserved pathway also controls planar polarity in many aspects of vertebrate development (Goodrich and Strutt, 2011; Wang and Nathans, 2007), including cell and tissue movements during gastrulation (Goto and Keller, 2002; Jessen et al., 2002; Takeuchi et al., 2003; Wallingford et al., 2001), the differentiation of stereocilia in mouse inner ear (Montcouquiol et al., 2003; Wang et al., 2005; Wang et al., 2006), the morphogenesis of the fur of mice (Chang and Nathans, 2013), kidney development (Babayeva et al., 2013; Goggolidou, 2014) and the polarity of the cilia in the respiratory epithelium (Vladar et al., 2012).

Early research placed the genes of the $f z /$ stan signaling pathway into three groups based on phenotype and epistatsis. The most upstream is called the PCP or core group and it includes frizzled $(f z)$, disheveled (dsh), prickle/spiny leg (pk/sple), Van Gogh(Vang) (also known as strabismus), starry night (stan) (also known as flamingo) and diego (dgo). The common features of the genes from this group are first, their protein products accumulate asymmetrically on either distal (Fz, Dsh and Dgo) (Axelrod, 2001; Das et al., 2004; Feiguin et al., 2001; Strutt, 2001), proximal (Vang and Pk) (Bastock et al., 2003; Jenny et al., 2003; Tree et al., 2002), or 
both edges of the wing cells (Stan) (Usui et al., 1999); second, the loss-of-function (lof) mutation of these genes share a similar mutant phenotype with majority of the cells forming single hair at more centralized location compared to the wild type (Figure 3B and 3B').

The group downstream of the PCP core group is the planar cell polarity effector (PPE) genes. Mutations in this group are epistatic to mutations in PCP core genes. The PPE includes inturned (in), fuzzy (fy) and fritz (frtz) (Collier et al., 2005; Wang et al., 2014; Wong and Adler, 1993) whose protein products are all asymmetrically localized on proximal side of wing cell (Adler et al., 2004; Collier et al., 2005; Strutt and Warrington, 2008; Wang et al., 2014). PPE mutants share the same common abnormal wing hair polarity pattern as the PCP genes but different in several aspects. First, many cells in PPE mutants form multiple (2-3) hairs (Figure $3 \mathrm{C}$ and $\left.3 \mathrm{C}^{\prime}\right)$. Second, hairs initiate alone the cell periphery, instead of a more centralized location. A variety of evidence demonstrate that the PPE genes function downstream of the PCP core genes. Lof mutations in PPE genes are epistatic to lof mutations in PCP core genes, and the functions of PPE proteins are not required for the asymmetric accumulation of PCP core proteins (Strutt, 2001; Usui et al., 1999; Wong and Adler, 1993). In contrast, the proximal accumulation of PPE proteins requires the proper function of and is instructed by PCP core group (Adler et al., 2004; Strutt and Warrington, 2008). Similar experimental results and genetic studies indicate that multiple-wing-hair $(m w h)$ functions downstream of the PPE group (Figure 3D and 3D') (Strutt and Warrington, 2008; Wong and Adler, 1993; Yan et al., 2008b).

\section{Protein interactions among PCP core}

A variety of data shows that there are at least two protein complexes formed within each wing cell, one on proximal side and another on distal side. Proteins encoded by PCP core genes accumulate asymmetrically in Drosophila wing cells. Vang and Pk accumulate proximally, Fz, 
Dsh and Dgo accumulate distally, and Stan accumulates on both the proximal and distal edges (Axelrod, 2001; Bastock et al., 2003; Feiguin et al., 2001; Shimada et al., 2001; Strutt, 2001; Tree et al., 2002; Usui et al., 1999).

Two types of interactions have been documented to play a role in the formation of these two protein domains. These are an intracellular negative feedback system and an intercellular positive feedback system. The intracellular negative interaction involves the cytoplasmic PCP proteins (Amonlirdviman et al., 2005; Tree et al., 2002). For example, interaction between Pk and Dsh inhibits the accumulation of Dsh on proximal side and a similar interaction inhibits the accumulation of $\mathrm{Pk}$ on distal side (Bastock et al., 2003). The intercellular feedback system involves interactions between transmembrane proteins juxtaposed on the proximal and distal edges of neighboring cells. There are arguments in the literature about the mechanism that underlies this. Interactions between $\mathrm{Fz}$ and Vang, perhaps in complex with Stan are thought to be important. Thus Fz on the distal side of one cell promotes the accumulation of Vang on the proximal side of its distal neighbor (and vice versa) (Chen et al., 2008; Strutt et al., 2008).This basic mechanism is thought to be responsible for the propagation of the polarity signal to neighboring cells and is a mechanism for correcting any cells that are out of alignment with their neighbors (Figure 4).

A third part of the system is due to the ability of the transmembrane proteins to recruit the cytoplasmic proteins to one side of the cell. For example, an interaction between $\mathrm{Fz}$ and Dsh leads to Dsh being recruited to the distal side (Wong et al., 2003) and an interaction between Vang and Pk leads to Pk being recruited to the proximal side (Bastock et al., 2003; Jenny et al., 2003).

\section{Planar cell polarity effectors (PPE) in Drosophila}


The PCP effectors in the $f z /$ stan pathway include in, $f y$ and $f r t z$, and they are responsible for regulating various important developmental processes during animal development. In Drosophila they function to couple the localization of the upstream genes to the cytoskeleton. WDPCP, mammalian homolog of Drosohila Frtz has been found to regulate the actin cytoskeleton in the mouse. The organization of actin filaments and focal contacts was markedly changed in wdpcp-deficient cells (Cui et al., 2013). The mammalian homologs of in and fy (intu and $f u z$ ) are required for ciliogenesis (Collier et al., 2005; Cui et al., 2013; Heydeck and Liu, 2011; Heydeck et al., 2009).

The proteins encoded by PPE are all cytoplasmic. There is a putative PDZ domain in N terminal half of In protein (Park et al., 1996; Yun et al., 1999), a coiled-coil domain and two WD40 repeats in Frtz protein (Collier et al., 2005). Fy interacts with both In and Frtz genetically, but no conserved domains have been found in it (Collier and Gubb, 1997; Collier et al., 2005; Wang et al., 2014). Mwh which is downstream of the PPEs, has a GTPase-binding domain (GBD) followed by a formin homology 3 (FH3) domain (Lu et al., 2010; Yan et al., 2008b).

Studies show Drosophila PPE proteins are recruited to the proximal edge of wing cells in a PCP core protein dependent manner (Adler et al., 2004; Strutt and Warrington, 2008). For example, in PCP core gene $f z$ or vang mutant clones, the asymmetric accumulation of In and Frtz are lost (Adler, 2012; Strutt and Warrington, 2008). In frtz or in mutant background, Mwh accumulation was decreased (Strutt and Warrington, 2008). However, the asymmetric accumulation of PCP core proteins is not altered by the mutations in PPE genes or $m w h$. All these results are consistent with the epistasis data that PPE genes are epistatic to PCP core genes, and $m w h$ is epistatic to both groups. In mammalian cells, Fuz (Drosophila Fy homolog) 
interacts with PCP core protein Dvl2 (Dishevelled-2, Dsh homolog) and recruits it to Rab8positive vesicles and to the basal body and cilium. In Drosophila, there was no data showing a physical interaction between PPE and PCP core proteins prior to this dissertation. As is described in chapter 3, I have uncovered several such interactions.

This thesis studies the functions of downstream PPE genes, in, fy and frtz in the $f z /$ stan signaling pathway and their relationships with upstream PCP core group. In chapter 2, I describe that although a variety of data support the hierarchy structure of $f z /$ stan signaling pathway, I have found that when over expressed the PPE proteins can alter both the subcellular location and level of accumulation of the upstream proteins. Thus, the epistatic relationship is context dependent. I further show that the PPE proteins interact physically and can modulate the accumulation of each other in wing cells. I also find that over expression of Frtz results in a marked delay in hair initiation suggesting that it has a separate role/activity in regulating the cytoskeleton that is not shared by other members of the group.

In Chapter 3, I describe my attempts to map the amino acid residues required for the interaction between In and Frtz WD40 repeats. I also describe the methods I used to generate in precise deletion $\left(i^{P D}\right)$ using CRISPR/Cas9 genome editing technique and found that Frtz has In- independent function in Drosophila wing cells. Mild overexpression of Frtz partially rescues In lof phenotype. I further demonstrate physical interactions between PPE proteins and PCP core protein Dsh. Using the yeast-two-hybrid and co immunoprecipitation assays, I found Dsh interacts with both Fy and Frtz, suggesting the asymmetric accumulation of PPE proteins are mediated by physical interactions with Dsh. Multiple locations in Frtz protein contribute to the interaction with Dsh. They are a 300 amino acids fragment on Frtz amino-terminus and a 100 amino acids fragment toward Frtz carboxyl-terminus. 
In chapter 4, I describe a newly generated $f r t z^{m N e o n \text { Green }}$ stock for studying the native Frtz protein activity in various tissues in Drosophila. Using the CRISPR/Cas 9 genome editing technique, I modified frtz gene in Drosophila genome by adding a green fluorescent protein mNeonGreen on its carboxyl terminus. This edited gene provides full rescue of $f r t z$ and is good for imaging the Frtz protein in various tissues, such as wing, thorax, abdomen and arista. Timelapse imaging data shows Frtz protein is preferentially distributed to discrete membrane subdomains ("puncta"), and the puncta is very dynamic at the subcellular level. 
Figure 1. Types of cell polarity. All epithelia feature an apical-basal polarity which is described as polarity orthogonal to the plane of the tissue. In addition, most epithelial tissue is also polarized within the plane of the epithelium, named planar cell polarity in which specialized structures are oriented within the plane of the epithelial sheet. The cartoon shows the Drosophila wing epithelia cells which are connected by adherens and septate junctions. 


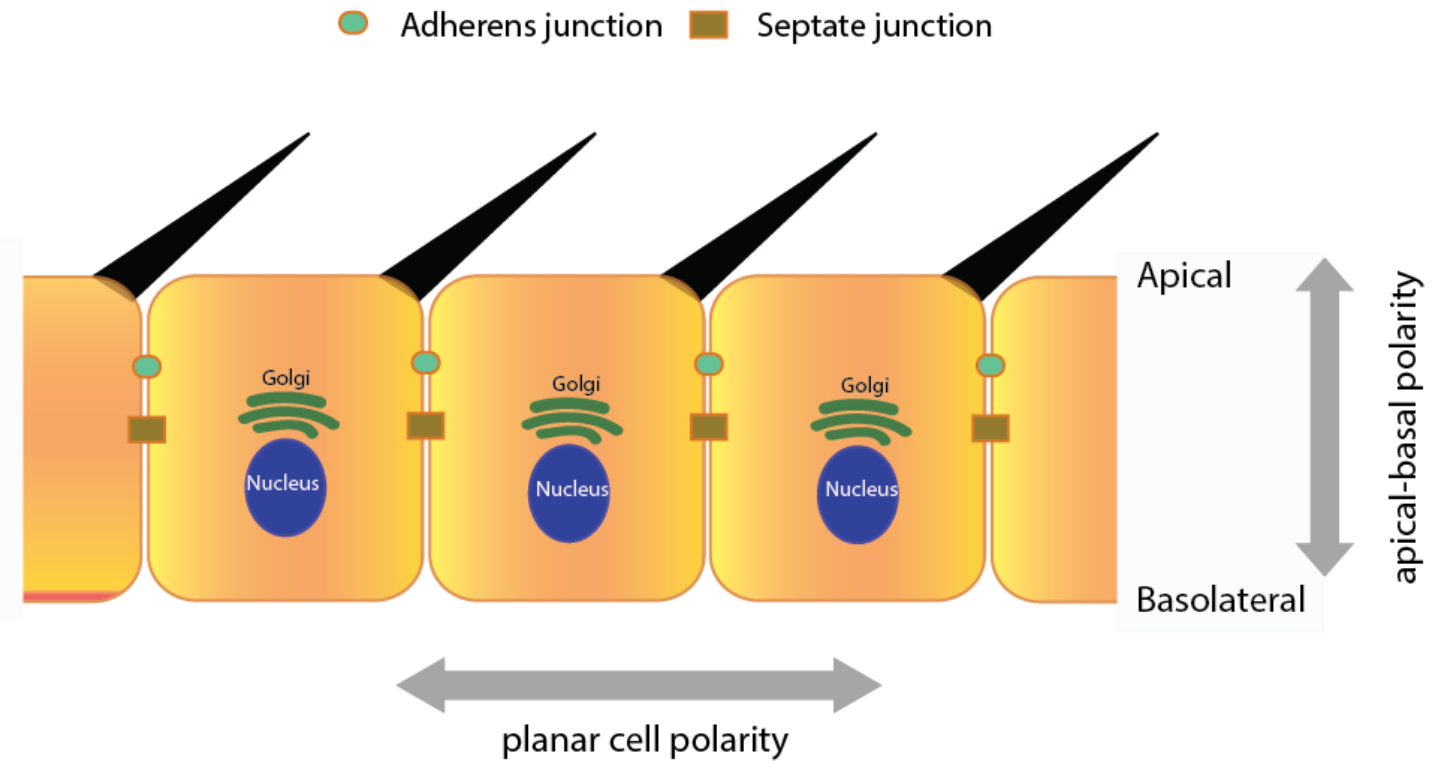


Figure 2. Various tissues in Drosophila show a PCP phenotype. A shows a wild-type wing. A' shows wing hair phenotype within the red rectangle area in A. B is the Drosophila thorax with well aligned sensory bristles. B' shows sensory bristles on the Drosophila femur. C shows Drosophila compound eye. A compound eye has a meshlike appearance because it consists of 750 tiny lens-capped optical units called ommatidia. Each ommatidium has its own cornea, lens, and photoreceptor cells for distinguishing brightness and color. C' is a cartoon of the adult retina with northern (green) and southern (red) hemisphere ommatidia in mirror image across the equator. Image $\mathrm{C}$ was obtained from the Kumar lab at Indiana University http://www.bio.indiana.edu/faculty/directory/profile.php?person=jkumar 

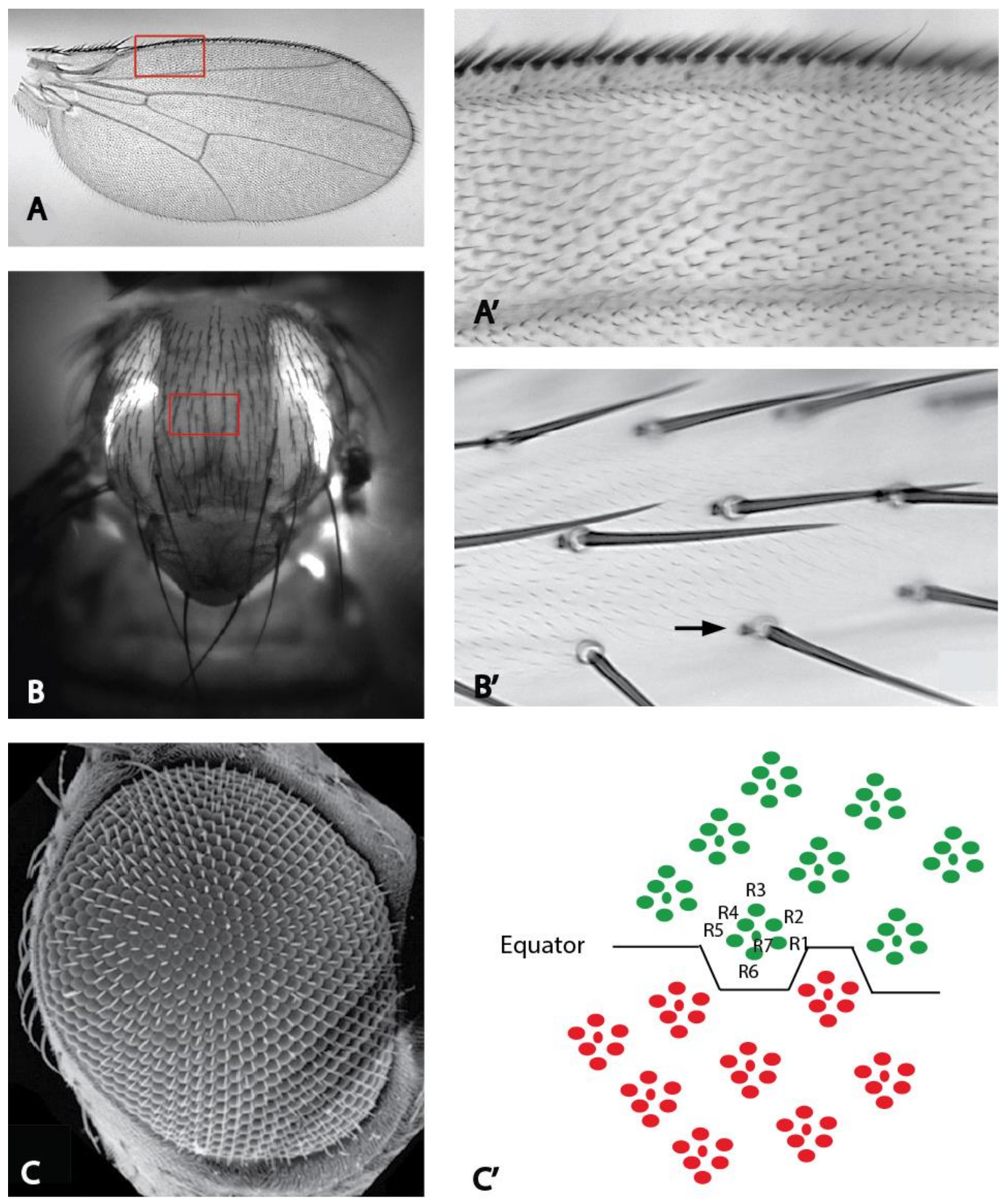
Figure 3. PCP genes lof phenotypes. A-D shows cartoons of wild-type and mutant wing cells. In wild-type cells, hairs are formed at the distal vertex (A). In cells mutant for PCP core genes, hairs form at a relatively central location (B). In cells mutant for PPE genes, multiple hairs form at an abnormal location on the cell periphery (C). In cells mutant for mwh genes, multiple hairs form at an abnormal location on the cell periphery (D). A'-D' shows wing hair phenotype for one mutant from each group. A' shows wild-type wing hair; B' shows the mutant phenotype of the PCP core gene $f z$; C' shows the mutant phenotype of the PPE gene in and D' is the mutant phenotype of $m w h$. 
A wild type

$A^{\prime}$

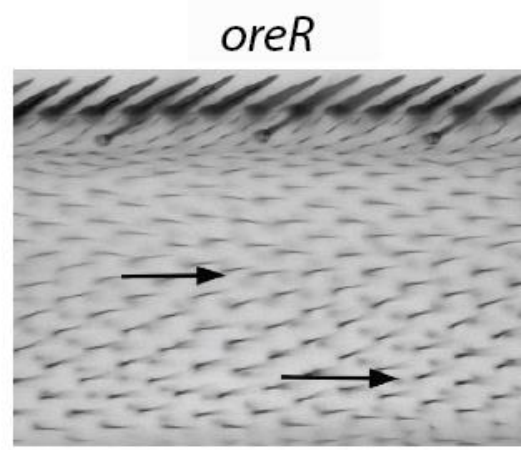

B $f z$, dsh,vang, stan,pk

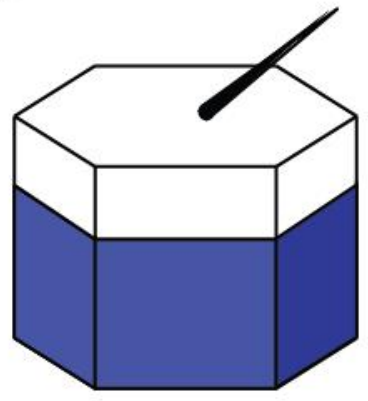

$B^{\prime}$

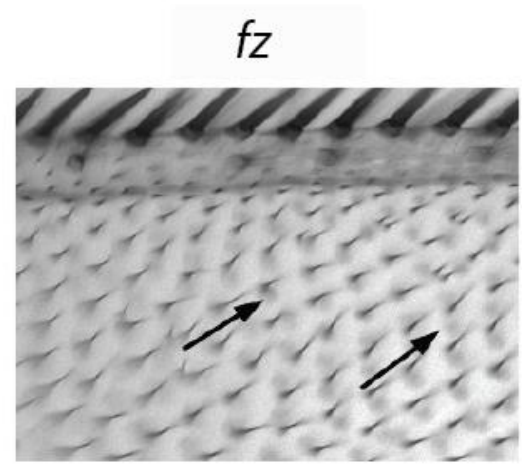

C in, fy, frtz

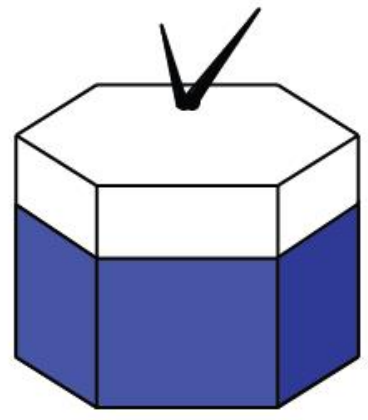

$C^{\prime}$

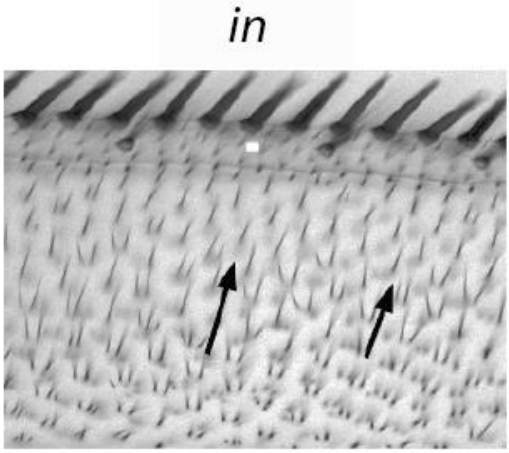

D mwh

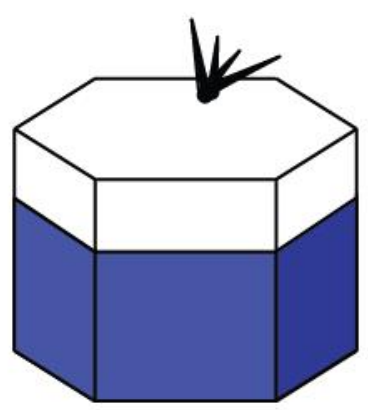

$D^{\prime}$

mwh

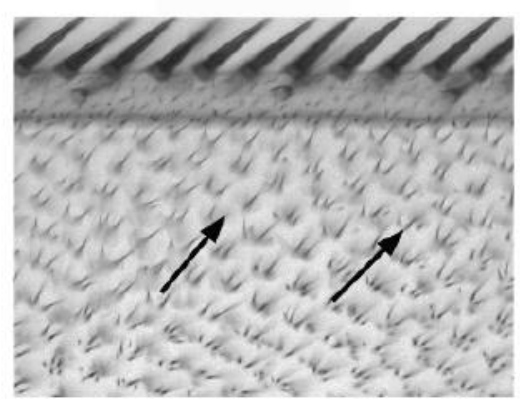


Figure 4. Protein interactions between PCP core proteins. There are two protein complexes within each wing cell, one on the proximal side (P) and another on the distal side (D). The proximal protein complex includes Stan, Vang and PK; the distal protein complex includes Stan, Fz, Dsh and Dgo. Two types of interaction were documentated, negative and positive interactions. The negative interactions occur between cytoplamic proteins $\mathrm{Pk}$, Dsh and Dgo within the same cell, and this prevents the proximally localized proteins accumulating distally and vice versa. The positive interactions function intercellularly and involve interactions between transmembrane proteins juxtaposed on the proximal and distal edges of neighboring cells. Fz on the distal edge of one cell promotes the accumulation of Vang on the proximal side of its distal neighbor (and vice versa). Also, the positive interactions occur between transmembrane proteins and cytoplasmic proteins. The positive interactions between Vang and $\mathrm{Pk}, \mathrm{Fz}$ and Dsh result in $\mathrm{Pk}$ accumulating proximally and Dsh accumulating distally. 


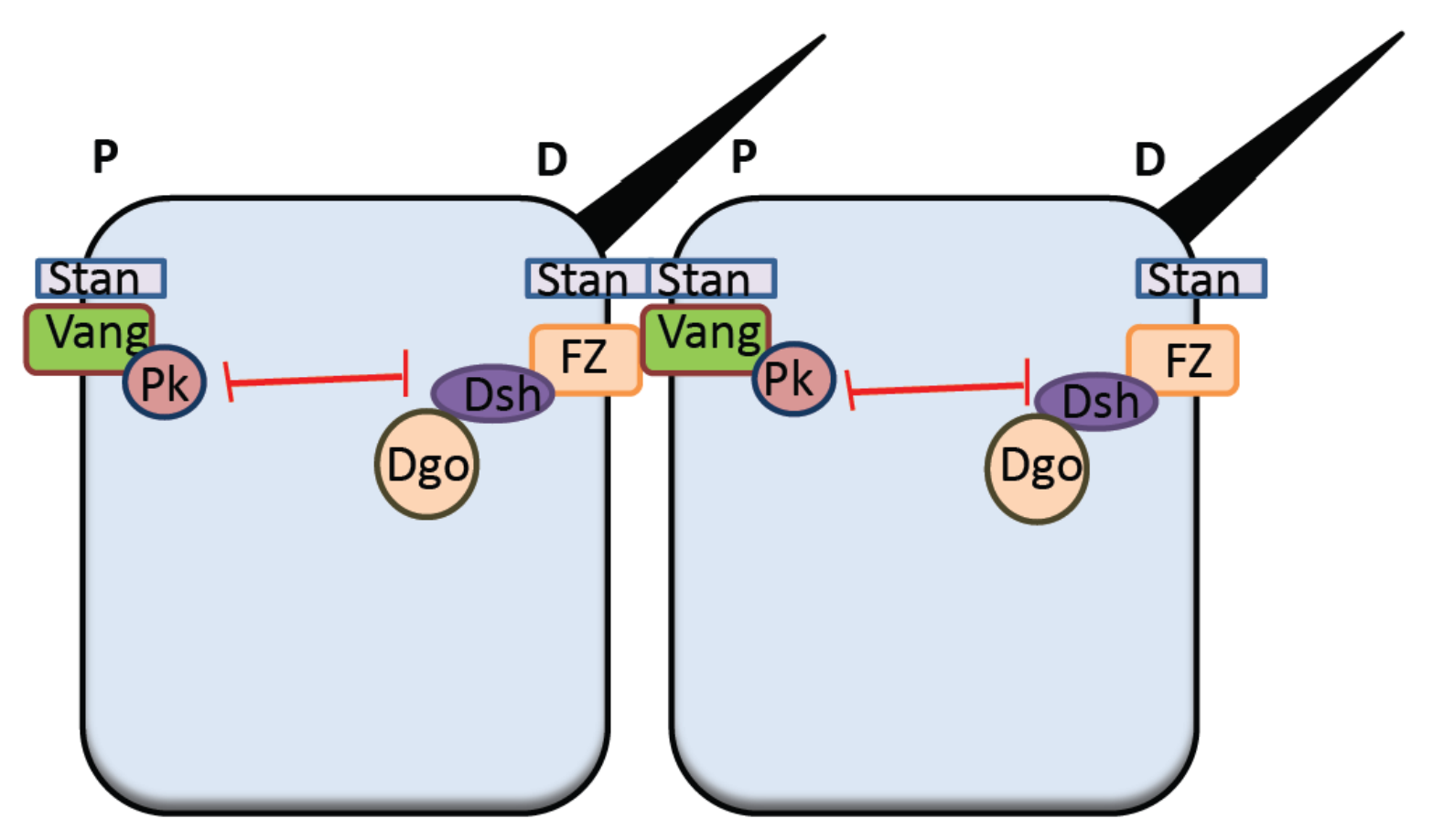




\section{Reference}

Adler, P.N. (2002). Planar signaling and morphogenesis in Drosophila. Dev Cell 2, 525-535.

Adler, P.N. (2012). The frizzled/stan pathway and planar cell polarity in the Drosophila wing. Curr Top Dev Biol 101, 1-31.

Adler, P.N., Charlton, J., and Liu, J. (1998). Mutations in the cadherin superfamily member gene

dachsous cause a tissue polarity phenotype by altering frizzled signaling. Development 125 , $959-968$.

Adler, P.N., Zhu, C., and Stone, D. (2004). Inturned localizes to the proximal side of wing cells under the instruction of upstream planar polarity proteins. Curr Biol 14, 2046-2051.

Axelrod, J.D. (2001). Unipolar membrane association of Dishevelled mediates Frizzled planar cell polarity signaling. Genes Dev 15, 1182-1187.

Babayeva, S., Rocque, B., Aoudjit, L., Zilber, Y., Li, J., Baldwin, C., Kawachi, H., Takano, T., and Torban, E. (2013). Planar cell polarity pathway regulates nephrin endocytosis in developing podocytes. J Biol Chem 288, 24035-24048. 
Bastock, R., Strutt, H., and Strutt, D. (2003). Strabismus is asymmetrically localised and binds to Prickle and Dishevelled during Drosophila planar polarity patterning. Development 130, 3007-3014.

Chae, J., Kim, M.J., Goo, J.H., Collier, S., Gubb, D., Charlton, J., Adler, P.N., and Park, W.J. (1999). The Drosophila tissue polarity gene starry night encodes a member of the protocadherin family. Development 126, 5421-5429.

Chang, H., and Nathans, J. (2013). Responses of hair follicle-associated structures to loss of planar cell polarity signaling. Proc Natl Acad Sci U S A 110, E908-917.

Collier, S., and Gubb, D. (1997). Drosophila tissue polarity requires the cell-autonomous activity of the fuzzy gene, which encodes a novel transmembrane protein. Development 124 , 4029-4037.

Collier, S., Lee, H., Burgess, R., and Adler, P. (2005). The WD40 repeat protein fritz links cytoskeletal planar polarity to frizzled subcellular localization in the Drosophila epidermis. Genetics 169, 2035-2045.

Cui, C., Chatterjee, B., Lozito, T.P., Zhang, Z., Francis, R.J., Yagi, H., Swanhart, L.M., Sanker, S., Francis, D., Yu, Q., et al. (2013). Wdpcp, a PCP protein required for ciliogenesis, regulates directional cell migration and cell polarity by direct modulation of the actin cytoskeleton. PLoS Biol 11, e1001720. 
Curtin, J.A., Quint, E., Tsipouri, V., Arkell, R.M., Cattanach, B., Copp, A.J., Henderson, D.J., Spurr, N., Stanier, P., Fisher, E.M., et al. (2003). Mutation of Celsr1 disrupts planar polarity of inner ear hair cells and causes severe neural tube defects in the mouse. Curr Biol 13, 1129-1133. Dabdoub, A., and Kelley, M.W. (2005). Planar cell polarity and a potential role for a Wnt morphogen gradient in stereociliary bundle orientation in the mammalian inner ear. J Neurobiol $64,446-457$.

Das, G., Jenny, A., Klein, T.J., Eaton, S., and Mlodzik, M. (2004). Diego interacts with Prickle and Strabismus/Van Gogh to localize planar cell polarity complexes. Development 131, 44674476.

Donoughe, S., and DiNardo, S. (2011). dachsous and frizzled contribute separately to planar polarity in the Drosophila ventral epidermis. Development 138, 2751-2759.

Feiguin, F., Hannus, M., Mlodzik, M., and Eaton, S. (2001). The ankyrin repeat protein Diego mediates Frizzled-dependent planar polarization. Dev Cell 1, 93-101.

Fichelson, P., and Gho, M. (2003). The glial cell undergoes apoptosis in the microchaete lineage of Drosophila. Development 130, 123-133.

Fischer, E., Legue, E., Doyen, A., Nato, F., Nicolas, J.F., Torres, V., Yaniv, M., and Pontoglio, M. (2006). Defective planar cell polarity in polycystic kidney disease. Nat Genet 38, 21-23. 
Gho, M., Bellaiche, Y., and Schweisguth, F. (1999). Revisiting the Drosophila microchaete lineage: a novel intrinsically asymmetric cell division generates a glial cell. Development 126 , 3573-3584.

Gho, M., and Schweisguth, F. (1998). Frizzled signalling controls orientation of asymmetric sense organ precursor cell divisions in Drosophila. Nature 393, 178-181.

Goggolidou, P. (2014). Wnt and planar cell polarity signaling in cystic renal disease. Organogenesis 10, 86-95.

Goodrich, L.V., and Strutt, D. (2011). Principles of planar polarity in animal development. Development 138, 1877-1892.

Goto, T., and Keller, R. (2002). The planar cell polarity gene strabismus regulates convergence and extension and neural fold closure in Xenopus. Dev Biol 247, 165-181.

Gubb, D., and Garcia-Bellido, A. (1982). A genetic analysis of the determination of cuticular polarity during development in Drosophila melanogaster. J Embryol Exp Morphol 68, 37-57.

Hamblet, N.S., Lijam, N., Ruiz-Lozano, P., Wang, J., Yang, Y., Luo, Z., Mei, L., Chien, K.R., Sussman, D.J., and Wynshaw-Boris, A. (2002). Dishevelled 2 is essential for cardiac outflow tract development, somite segmentation and neural tube closure. Development 129, 5827-5838. 
Henderson, D.J., Conway, S.J., Greene, N.D., Gerrelli, D., Murdoch, J.N., Anderson, R.H., and Copp, A.J. (2001). Cardiovascular defects associated with abnormalities in midline development in the Loop-tail mouse mutant. Circ Res 89, 6-12.

Henderson, D.J., Phillips, H.M., and Chaudhry, B. (2006). Vang-like 2 and noncanonical Wnt signaling in outflow tract development. Trends Cardiovasc Med 16, 38-45.

Heydeck, W., and Liu, A. (2011). PCP effector proteins inturned and fuzzy play nonredundant roles in the patterning but not convergent extension of mammalian neural tube. Dev Dyn 240, 1938-1948.

Heydeck, W., Zeng, H., and Liu, A. (2009). Planar cell polarity effector gene Fuzzy regulates cilia formation and Hedgehog signal transduction in mouse. Dev Dyn 238, 3035-3042.

Jenny, A., Darken, R.S., Wilson, P.A., and Mlodzik, M. (2003). Prickle and Strabismus form a functional complex to generate a correct axis during planar cell polarity signaling. EMBO J 22, 4409-4420.

Jessen, J.R., Topczewski, J., Bingham, S., Sepich, D.S., Marlow, F., Chandrasekhar, A., and Solnica-Krezel, L. (2002). Zebrafish trilobite identifies new roles for Strabismus in gastrulation and neuronal movements. Nat Cell Biol 4, 610-615. 
Keller, R., Davidson, L., Edlund, A., Elul, T., Ezin, M., Shook, D., and Skoglund, P. (2000). Mechanisms of convergence and extension by cell intercalation. Philos Trans R Soc Lond B Biol Sci 355, 897-922.

Klingensmith, J., Nusse, R., and Perrimon, N. (1994). The Drosophila segment polarity gene dishevelled encodes a novel protein required for response to the wingless signal. Genes Dev 8 , 118-130.

Lu, B., Usui, T., Uemura, T., Jan, L., and Jan, Y.N. (1999). Flamingo controls the planar polarity of sensory bristles and asymmetric division of sensory organ precursors in Drosophila. Curr Biol 9, 1247-1250.

Lu, Q., Yan, J., and Adler, P.N. (2010). The Drosophila planar polarity proteins inturned and multiple wing hairs interact physically and function together. Genetics $185,549-558$.

Maung, S.M., and Jenny, A. (2011). Planar cell polarity in Drosophila. Organogenesis 7, 165179.

Montcouquiol, M., Rachel, R.A., Lanford, P.J., Copeland, N.G., Jenkins, N.A., and Kelley, M.W. (2003). Identification of Vangl2 and Scrb1 as planar polarity genes in mammals. Nature 423, $173-177$.

Park, W.J., Liu, J., Sharp, E.J., and Adler, P.N. (1996). The Drosophila tissue polarity gene inturned acts cell autonomously and encodes a novel protein. Development 122, 961-969. 
Price, M.H., Roberts, D.M., McCartney, B.M., Jezuit, E., and Peifer, M. (2006). Cytoskeletal dynamics and cell signaling during planar polarity establishment in the Drosophila embryonic denticle. J Cell Sci 119, 403-415.

Repiso, A., Saavedra, P., Casal, J., and Lawrence, P.A. (2010). Planar cell polarity: the orientation of larval denticles in Drosophila appears to depend on gradients of Dachsous and Fat. Development 137, 3411-3415.

Singh, A., Chan, J., Chern, J.J., and Choi, K.W. (2005). Genetic interaction of Lobe with its modifiers in dorsoventral patterning and growth of the Drosophila eye. Genetics 171, 169-183.

Strutt, D., and Warrington, S.J. (2008). Planar polarity genes in the Drosophila wing regulate the localisation of the FH3-domain protein Multiple Wing Hairs to control the site of hair production. Development 135, 3103-3111.

Strutt, D.I. (2001). Asymmetric localization of frizzled and the establishment of cell polarity in the Drosophila wing. Mol Cell 7, 367-375.

Takeuchi, M., Nakabayashi, J., Sakaguchi, T., Yamamoto, T.S., Takahashi, H., Takeda, H., and Ueno, N. (2003). The prickle-related gene in vertebrates is essential for gastrulation cell movements. Curr Biol 13, 674-679. 
Taylor, J., Abramova, N., Charlton, J., and Adler, P.N. (1998). Van Gogh: a new Drosophila tissue polarity gene. Genetics 150, 199-210.

Tomlinson, A., and Struhl, G. (1999). Decoding vectorial information from a gradient: sequential roles of the receptors Frizzled and Notch in establishing planar polarity in the Drosophila eye. Development 126, 5725-5738.

Tree, D.R., Shulman, J.M., Rousset, R., Scott, M.P., Gubb, D., and Axelrod, J.D. (2002). Prickle mediates feedback amplification to generate asymmetric planar cell polarity signaling. Cell 109, 371-381.

Usui, T., Shima, Y., Shimada, Y., Hirano, S., Burgess, R.W., Schwarz, T.L., Takeichi, M., and Uemura, T. (1999). Flamingo, a seven-pass transmembrane cadherin, regulates planar cell polarity under the control of Frizzled. Cell 98, 585-595.

Vladar, E.K., Bayly, R.D., Sangoram, A.M., Scott, M.P., and Axelrod, J.D. (2012). Microtubules enable the planar cell polarity of airway cilia. Curr Biol 22, 2203-2212.

Wallingford, J.B., Fraser, S.E., and Harland, R.M. (2002). Convergent extension: the molecular control of polarized cell movement during embryonic development. Dev Cell 2, 695-706.

Wallingford, J.B., Rowning, B.A., Vogeli, K.M., Rothbacher, U., Fraser, S.E., and Harland, R.M. (2000). Dishevelled controls cell polarity during Xenopus gastrulation. Nature 405, 81-85. 
Wallingford, J.B., Vogeli, K.M., and Harland, R.M. (2001). Regulation of convergent extension in Xenopus by Wnt5a and Frizzled-8 is independent of the canonical Wnt pathway. Int J Dev Biol 45, 225-227.

Wang, J., Mark, S., Zhang, X., Qian, D., Yoo, S.J., Radde-Gallwitz, K., Zhang, Y., Lin, X., Collazo, A., Wynshaw-Boris, A., et al. (2005). Regulation of polarized extension and planar cell polarity in the cochlea by the vertebrate PCP pathway. Nat Genet 37, 980-985.

Wang, Y., Guo, N., and Nathans, J. (2006). The role of Frizzled3 and Frizzled6 in neural tube closure and in the planar polarity of inner-ear sensory hair cells. J Neurosci 26, 2147-2156.

Wang, Y., and Nathans, J. (2007). Tissue/planar cell polarity in vertebrates: new insights and new questions. Development 134, 647-658.

Wang, Y., Yan, J., Lee, H., Lu, Q., and Adler, P.N. (2014). The proteins encoded by the Drosophila Planar Polarity Effector genes inturned, fuzzy and fritz interact physically and can re-pattern the accumulation of "upstream" Planar Cell Polarity proteins. Dev Biol 394, 156-169.

Wolff, T., and Rubin, G.M. (1998). Strabismus, a novel gene that regulates tissue polarity and cell fate decisions in Drosophila. Development 125, 1149-1159.

Wong, L.L., and Adler, P.N. (1993). Tissue polarity genes of Drosophila regulate the subcellular location for prehair initiation in pupal wing cells. J Cell Biol 123, 209-221. 
Yan, J., Huen, D., Morely, T., Johnson, G., Gubb, D., Roote, J., and Adler, P.N. (2008). The multiple-wing-hairs gene encodes a novel GBD-FH3 domain-containing protein that functions both prior to and after wing hair initiation. Genetics $180,219-228$.

Yang, C.H., Axelrod, J.D., and Simon, M.A. (2002). Regulation of Frizzled by fat-like cadherins during planar polarity signaling in the Drosophila compound eye. Cell 108, 675-688.

Yun, U.J., Kim, S.Y., Liu, J., Adler, P.N., Bae, E., Kim, J., and Park, W.J. (1999). The inturned protein of Drosophila melanogaster is a cytoplasmic protein located at the cell periphery in wing cells. Dev Genet 25, 297-305.

Zeidler, M.P., Perrimon, N., and Strutt, D.I. (1999). The four-jointed gene is required in the Drosophila eye for ommatidial polarity specification. Curr Biol 9, 1363-1372.

Zheng, L., Zhang, J., and Carthew, R.W. (1995). frizzled regulates mirror-symmetric pattern formation in the Drosophila eye. Development 121, 3045-3055. 


\section{Chapter 2}

The proteins encoded by the Drosophila Planar Polarity Effector genes inturned, fuzzy and fritz interact physically and can re-pattern the accumulation of "upstream" Planar Cell Polarity proteins

Ying Wang, Jie Yan, Haeryun Lee, Paul N. Adler 


\begin{abstract}
The frizzled/starry night pathway regulates planar cell polarity in a wide variety of tissues in many types of animals. It was discovered and has been most intensively studied in the Drosophila wing where it controls the formation of the array of distally pointing hairs that cover the wing. The pathway does this by restricting the activation of the cytoskeleton to the distal edge of wing cells. This results in hairs initiating at the distal edge and growing in the distal direction. All of the proteins encoded by genes in the pathway accumulate asymmetrically in wing cells. The pathway is a hierarchy with the planar cell polarity (PCP) genes (aka the core genes) functioning as a group upstream of the planar polarity effector (PPE) genes which in turn function as a group upstream of multiple wing hairs. Upstream proteins, such as Frizzled accumulate on either the distal and/or proximal edges of wing cells. Downstream PPE proteins accumulate on the proximal edge under the instruction of the upstream proteins. A variety of types of data supports this hierarchy, however, we have found that when over expressed the PPE proteins can alter both the subcellular location and level of accumulation of the upstream proteins. Thus, the epistatic relationship is context dependent. We further show that the PPE proteins interact physically and can modulate the accumulation of each other in wing cells. We also find that over expression of Frtz results in a marked delay in hair initiation suggesting that it has a separate role/activity in regulating the cytoskeleton that is not shared by other members of the group.
\end{abstract}




\section{Introduction}

The epidermis of many animals is polarized within the plane of the tissue. This tissue planar cell polarity (PCP) is dramatic in the cuticle of insects such as Drosophila, which is decorated with arrays of hairs and sensory bristles. Tissue polarity in Drosophila is under the control of the frizzled (fz)/starry night (stan) signaling pathway and in recent years it has become clear that this conserved pathway also controls planar polarity in many aspects of vertebrate development (Goodrich and Strutt, 2011; Wang and Nathans, 2007) including cell and tissue movements during gastrulation (Goto and Keller, 2002; Jessen et al., 2002; Takeuchi et al., 2003; Wallingford et al., 2001), the differentiation of stereocilia in the inner ear (Montcouquiol et al., 2003; Wang et al., 2005; Wang et al., 2006), the morphogenesis of the fur of mice (Chang and Nathans, 2013), kidney development (Babayeva et al., 2013; Goggolidou, 2014) and the polarity of the cilia in the respiratory epithelium (Vladar et al., 2012).

Genetic studies focused on the fly wing led to the identification and characterization of a number of genes essential for PCP that comprise the $f z /$ stan signaling/signal transduction pathway (Chae et al., 1999; Das et al., 2004; Gubb and Garcia-Bellido, 1982; Held et al., 1986; Taylor et al., 1998; Usui et al., 1999; Wolff and Rubin, 1998; Wong and Adler, 1993). These genes consist of three phenotypic groups that are also epistasis groups (Wong and Adler, 1993). They are the PCP genes (aka, core group), the PPE (Planar Polarity Effecter) genes and the multiple wing hairs ( $m w h)$ gene. frizzled $(f z)$, disheveled (dsh), prickle/spiny leg (pk/sple), Van Gogh (Vang) (aka strabismus), starry night (stan) (aka flamingo) and diego (dgo) are all members of the PCP group. A distinctive feature of these genes is that their protein products accumulate asymmetrically on the distal (Fz, Dsh and Dgo) (Axelrod, 2001; Das et al., 2004; Feiguin et al., 2001; Strutt, 2001), proximal (Vang, Pk) (Bastock et al., 2003; Jenny et al., 2003; 
Tree et al., 2002) or both distal and proximal (Stan) edges of wing cells (Usui et al., 1999). These proteins are a functional group and are co-requirements for the asymmetric accumulation of the others.

The planar polarity effecter (PPE) group includes inturned (in), fuzzy (fy) and fritz (frtz). The abnormal polarity patterns seen in PPE mutants are similar to those seen in PCP mutants but PPE mutants differ in several ways from PCP mutants (Collier and Gubb, 1997; Collier et al., 2005; Park et al., 1996; Wong and Adler, 1993). The vast majority of PCP mutant wing cells form a single hair at a relatively central location on the apical surface, while in contrast many PPE mutant wing cells form 2 or 3 hairs at abnormal locations along the cell periphery. A variety of evidence argues that the PPE genes function downstream of the PCP genes. Loss of function mutations in PPE genes are epistatic to mutations in PCP genes and the function of the PPE genes is not required for the asymmetric accumulation of PCP proteins (Strutt, 2001; Usui et al., 1999; Wong and Adler, 1993). In contrast, the proximal accumulation of the PPE proteins requires the function of and is instructed by PCP genes (Adler et al., 2004; Strutt and Warrington, 2008). Similar experimental results indicate that $m w h$ functions downstream of the PPE genes (Strutt and Warrington, 2008; Wong and Adler, 1993; Yan et al., 2008b).

The PPE genes share almost identical mutant phenotypes, double null mutants do not show a stronger phenotype and the genes show strong genetic interactions (Adler et al., 2004; Collier et al., 2005; Wong and Adler, 1993). For example, any double mutant combination of weak PPE alleles results in a strong PPE mutant phenotype (Collier et al., 2005). These observations suggest that the PPE genes function as a unit. Consistent with this hypothesis all three are co-requirements for the proximal accumulation of the others and the level of In and 
Frtz are dramatically reduced when animals are mutant for another PPE gene (e.g. fy) (Adler et al., 2004; Strutt and Warrington, 2008). We report here that these proteins can be coimmunoprecipated in a variety of experimental conditions and that In interacted with both Fy and Frtz in the yeast two hybrid system (Lee and Adler, 2002; Yan et al., 2008b). We also observed that the over expression (oe) of one PPE protein could affect the accumulation of a second. Our data argue these proteins form a protein complex that functions in wing tissue polarity.

One of the hallmarks of the PCP genes is that over expression (oe) leads to a gain of function (gof) hair polarity phenotype that is superficially similar to the loss of function phenotype (Axelrod et al., 1998; Boutros et al., 1998; Feiguin et al., 2001; Gubb et al., 1999; Krasnow and Adler, 1994; Usui et al., 1999). This appears to be due to a loss of their asymmetric distribution, perhaps due to saturating binding sites. In contrast the oe of In or Fy did not lead to a gof phenotype even though that led to the loss or degradation of their asymmetric accumulation. A possible explanation for these results is that only properly localized protein is active. Alternatively, activity could require that the proteins are in the proper protein complex and the amount of the other component(s) is limiting when only one is over expressed. Interestingly, we found that oe of In and Fy together resulted in a relatively weak but consistent gof phenotype. This is consistent with the latter hypothesis. We also found that the oe of frtz by itself led to a gof planar polarity phenotype. What could be the mechanism by which the oe of PPE proteins can alter hair polarity? One possibility is that the oe of PPE proteins uncouples the site of hair initiation from the side of the cell where Fz, Dsh and Dgo accumulate, much as loss of function mutations in PPE genes do. Another possibility is that the oe of the PPE proteins could re-pattern the accumulation of the upstream PCP proteins. This seemed less 
likely as it requires putative downstream proteins to affect the localization of upstream proteins. Surprisingly, we found the latter hypothesis was correct. This establishes that the PPE proteins functioning downstream of the PCP proteins is context dependent and blurs the line between the two groups of genes. 


\section{Materials and methods}

\section{Fly genetics}

All flies were raised at $25^{\circ} \mathrm{C}$ unless otherwise stated. Mutant stocks were either obtained from the Bloomington Drosophila stock center at Indiana University, from the VDRC, generated in our lab or were generous gifts from J. Axelrod, D. Strutt, or T. Uemura. The FLP/FRT technology was used to generate genetics mosaics. To direct transgene expression, we used the Gal4/UAS system (Brand and Perrimon, 1993). For temperature shift experiments the

relevant animals were collected as white pre-pupae, placed into fresh food vials and moved to different temperatures at the desired time. Several of the genes used in this paper have multiple names. In line with FlyBase usage we use starry night instead of flamingo and Van Gogh instead of strabismus.

\section{Transgenes and antibodies}

We attempted to determine if Frtz and Fy, like In were localized to the proximal side of wing cells by raising antibodies against these two proteins. The antibodies generated were able to specifically detect over-expressed proteins but were not able to detect the endogenous proteins. As an alternative we constructed transgenes that encoded tagged versions of these genes. For $f y$ we generated GFP, Flag and Flag-Ollas tagged transgenes driven by the following promoter/regulatory sequences: UAS, hsp70, armadillo, ubiquitin and the endogenous fy promoter. All of these constructs completely rescued a fy null allele. Immunostaining of the tagged Fy proteins did not yield consistent data that Fy accumulated in the stereotypic zig zag pattern, although we saw this on occasion. Strutt and colleagues using a different construct found Fy to be asymmetrically localized (Strutt and Warrington, 2008). 
We also generated transgenic flies that expressed myc-Frtz-GFP from the ubiquitin promoter (ubi-Myc-Frtz-GFP) (Sekelsky et al., 1995). These transgenes provided complete rescue of frtz phenotypic null alleles and in experiments using them, we determined that Frtz was localized to the proximal side of wing cells. In z sections we found that Frtz was localized near the adherens junctions as is the case for other proteins involved in tissue polarity. These results confirm those previously published by Strutt and colleagues (Strutt and Warrington, 2008). We also generated transgenic lines that expressed frtz, myc-Frtz or Frtz-GFP under the direction of UAS. For reasons that are unclear, the myc-frtz transgene drove expression at a lower level than the other two and did not result in a gain of function phenotype. Perhaps this is due to the amino terminal tag lowering protein accumulation. For in, we used previously described transgenes that express HA-In driven by UAS (Park et al., 1996).

A surprising feature of the PPE UAS transgenes is that they often provided substantial genetic rescue in the absence of a GAL4 driver or in regions outside of the normal domain of Gal4 expression (Park et al., 1996). The degree of rescue was more complete for in and $f y$ than frtz and varied as a function of body region and insert. For example, rescue was usually not seen for the abdominal bristle polarity phenotype. The rescue is likely due to enhancer trapping driving expression of the transgenes. In some cases (for example for a frtz-GFP insert), we were able to detect this by immunostaining. Since we have not seen equivalent rescue of mutant wing phenotypes with other UAS-transgenes (e.g. UAS-mwh, UAS-trc, UAS-fz) we suggest that PPE function can be provided by relatively low levels of protein.

In our initial experiments standard $\mathrm{P}$ element vectors were used that resulted in relatively random insertions (Klemenz et al., 1987; Pirrotta, 1988). Later experiments utilized 
phi-C3linsertion vectors and insertion sites (Groth et al., 2004). For UAS-constructs we used the attp2 and attp40 landing sites (Bischof et al., 2007).

\section{Immunostaining}

A standard paraformaldehyde fixation and staining procedure was used (Adler et al., 2004).

\section{Plasmid constructs}

The full length frtz cDNA with a Myc tag at its N-terminus and the full length $f y$ cDNA with both OLLAS and FLAG tags at its C-terminus were generated by PCR and subcloned into pPUAST-attB vector. The full length frtz cDNA with a GFP tag on its C-terminus was subcloned into pPUM6 (Jeff Sekelsky's Lab) using EcoRI and SacII sites.

The following primers were used in the constructions:

PUAST-attB-(myc) $)_{6}$-frtz (from KpnI to XbaI)

5' primer: GAGGGTACCCCACCATGGGGCAGGGAT

3' primer: CTAGTCTAGACTAGACCACGCCGAAGTGGACCACC

PUAST-fy-FLAG-OLLAS (from XhoI to XbaI)

5'primer: CCGCTCGAGATGTCCATCTATTTGTTATGTTTGACAACAAACGGCGGATTG

3'primer:TGCTCTAGACTACTTGCCCATCAACCTAGGTCCCAATTCATTCGCAAA

GCCACT 
PUM6-frtz-GFP (from EcoRI to SacII)

5' primer: CCGGAATTCAATGCTGCTCAGCGAGAC

3' primer: TCCCCGCGGCTACTTGTACAGCTCGTCCATG

The UAS-HA-in construct was described previously (Lu et al., 2010).

\section{Construct subcloning for two-hybrid assays}

Full-length of inturned cDNA was subcloned into pGADT7 from NdeI-BamHI. The following primers were used:

Inturn-th5: TCTAGGGAATTTCCATATGCGCAAATCGCCGGCCAG;

Inturn-th3: GATCGCGGATCCATGTCATCCCATTGAGAAGAAGGA.

Full-length of fuzzy or fritz cDNA was subcloned into $p G B K T 7$ from NcoI-BamHI or NdeI-EcoRI respectively. The following primers were used:

pGBKT7-5': CATGCCATGGAGATGTCCATCTATTTGTTATG

pGBKT7-3': CGCGGATCCTTATCACCAACATACTGACTTC

5' frtz-hybrid: GGGAATTCCATATGCTGCTCAGCGAGACC

3'fritz-hybrid: CCGGAATTCTTATTAGACCACGCCGAAGTGGA

To get truncated forms of fritz cDNA, we used the following primers and the PCR products were subcloned into $p G B K T 7$ from NdeI-EcoRI sites. We used the same 5'fritz-hybrid 
primer and DBD-frtzN-3': CCGGAATTCTTAGTGCGACAGATCCAGCAAG to amplify the N-terminus of fritz cDNA (encoding 1-400 amino acids); we used DBD-frtzC-5': GGGAATT CCATATGTACTTCGTGGCCCAGCCA and the same 3'frtz-hybrid primer to amplify the Cterminus of fritz (encoding 401-951 amino acid). 5'frtz-hybrid primer and Dfrtz200-3': CCGG AATTCTTAAACTGTGAGGTGGCGG were used to amplify the fritz cDNA encoding 1-200 amino acids; Dfrzt400-5': GGGAATTCCATATGAACGCCAGCTTC GATC and 3'fritz-hybrid primer were used to PCR frtz cDNA encoding 201-400 amino acids.

To Amplify the frtz cDNA encoding WD40 repeats, the following primers were used: DBDFrtz-WD5:GGAATTCCATATGCAGATCTGCTCCTTTGCCTTC; DBD-Frtz-WD3: CG GAATTCTTACAGCTGATGGCCAATGGTG.

To examine interactions between truncated forms of Inturned and Fuzzy or Fritz, fulllength of fuzzy or fritz cDNA was subcloned into $p G A D T 7$ from NcoI-BamHI or NdeI-EcoRI respectively. We used the same pGBKT7-5', pGBKT7-3', 5'frtz-hybrid and 3'frtz-hybrid primers as described before. Truncated forms of inturned cDNA were generated by PCR and subcloned into $p G B K T 7$ from NdeI-BamHI. We used the same Inturn-th5' primer and inNterm1-3': CGCGGATCCTCACAAGATCACGGCCAGACTC to amplify the N-terminal part of Inturned (1-550 amino acid); we used in-Cterm1-5': GGGAATTCCATATGAAGATCTTC GATGCTCCAG and the same Inturn-th3' primer to amplify the C-terminal part of Inturned (551-869 amino acid). The fragment encoding Inturned 551-731 amino acid was cut from $p G B K T$-inC (551-869 aa) construct by restriction enzymes NdeI and EcoRI and inserted into $p G B K T 7$ or $p G A D T 7$ from the same sites. We used AD-in-731-5': GGAATTCCATATGGTTC TGCACTACGTCT ACA and the same Inturn-th3' primer to amplify inturned cDNA encoding 
731-869 amino acid, the PCR product was subcloned into $p G B K T 7$ or $p G A D T 7$ from NdeIBamHI.

For the yeast two hybrid assays, we used yeast strain AH109 and Matchmaker TwoHybrid System 3 from Clontech. Briefly, full length or truncated cDNA was subcloned into $p G A D T 7$ or $p G B K T 7$ by specific restriction enzyme sites. To test the interaction of defined protein partners, $p G A D T 7$ and $p G B K T 7$ plasmids, with each encoding one of the two protein partners were co-transformed into yeast. Transformed yeasts were selected by growing on twomarker dropout medium (SD/-Leu/-Trp). Colonies from previous dropout medium were transferred to a four-marker dropout medium (SD/-Ade/-His/-Leu/-Trp) with X-alpha-Gal. Only colonies growing on four-marker plates were considered as positive colonies.

\section{Antibodies}

Monoclonal anti-armadillo and anti-Flamingo antibodies were obtained from Developmental Studies Hybridoma Bank at the University of Iowa. Anti-HA, anti-Flag and anti-Myc antibodies were obtained from Cell Signaling Technology. Anti-Ollas monoclonal antibody was purchased from Novus Biologicals. Anti-GFP antibody was obtained from Molecular Probes. Alexa 488- and Alexa 568-conjugated secondary antibodies were purchased from Molecular Probes. Alexa 568 and 647 phalloidin were purchased from Molecular Probes. Anti-Pk antibodies were kindly provided by Drs. J. Axelrod and D. Strutt. Anti-Frtz antibody was generated in rats using a Frtz protein made in E. coli and his tagged to facilitate purification. A similar strategy was used to obtain an anti-Fy antibody. In both cases the expressed proteins were found in inclusion bodies and solubilized, and we do not know if they were in a native conformation. Neither antibody was able to detect the endogenous protein. 


\title{
Coimmunoprecipitation and western blotting
}

The following genotypes were used in these experiments:

\author{
w; ptc-Gal4/UAS-HA-in UAS-fy-GFP;UAS-frtz-GFP \\ w; ptc-Gal4/UAS-HA-in; UAS-frtz-GFP \\ w; ptc-Gal4/ UAS-fy-GFP;UAS-frtz-GFP \\ w; ptc-Gal4/UAS-HA-in; UAS-(myc) $)_{6}-f r t z$ \\ w; ptc-Gal4/UAS-HA-in UAS-fy-GFP \\ w; ptc-Gal4/UAS-fy-GFP; UAS-(myc) $)_{6}-f r t z$ \\ w; ptc-Gal4/UAS-HA-in; UAS-fy-FLAG-OLLAS \\ w; ptc-Gal4/UAS-fy-FLAG-OLLAS;UAS-(myc) ${ }_{6}-f r t z$ \\ whs-flp; UAS-HA-in UAS-fy-GFP
}

Co-immunoprecipitation experiments were done in a variety of different ways. The most common procedure was as follows. 150-200 wing discs of transgenic flies were dissected from third instar larvae and homogenized in pre-chilled lysis buffer containing protease inhibitors. The extract was then spun to remove cell debris. Protein A agarose beads (Roche) were added to the extract and incubated for at least $3 \mathrm{~h}$ to reduce the background of non-specific binding. The extract was spun again and 7-10 $\mu 1$ desired antibody was added to the supernatant and incubated for 3-4 h. New protein A agarose beads were then added and the sample was 
incubated overnight. The beads were pelleted and washed. Protein was released from the beads and analyzed by standard western blot procedures. In other experiments we used as starting material S2 cells, whole larvae or larval "heads" (these were dissected anterior sections of larvae that contained the brain, imaginal discs, salivary glands, anterior larval epidermis and other tissues). Similar results were obtained from all three sources.

\section{Imaging}

Adult wings were mounted in Euparal and bright field images obtained using a Spot RT camera and a Zeiss Axiskop II microscope. In most cases a 40X Apochromatic objective was used (oil, 1.4 NA). When wings were not flat enough to allow a good final image from a single optical plane a $\mathrm{Z}$ stack was obtained using Metamorph and a final image was obtained either using a minimal projection (most cases) or by using an Image $\mathbf{J}$ extended focus plug in. In a few very difficult preparations a $\mathrm{Z}$ stack was obtained and prior to carrying out the minimal projection for the dorsal wing hairs the ventral hairs were erased manually from some optical planes. Quantitation of images was accomplished using ImageJ.

Confocal images of immunostained pupal wings were obtained using either a Zeiss 510 or a Leica SP5 WLL confocal microscope. Final panels were assembled using Adobe Photoshop or Microsoft PowerPoint. 


\section{Results}

Many of the experiments reported in this paper used transgenes to express PPE proteins. Most of these have not been described previously and details are provided in the Methods and Materials. All of the tagged (and untagged) transgenes used were able to provide complete rescue of null alleles when expressed at appropriate levels (e.g. using the ubiquitin promoter/enhancer to drive expression). Tagged In and Frtz proteins encoded by the transgenes localized to the proximal side of wing cells as the endogenous proteins do (Fig. S1) (Adler et al., 2004; Strutt and Warrington, 2008). This was also reported to be the case for Fy (Strutt and Warrington, 2008). Using tagged transgenes we have on occasion seen evidence of asymmetric Fy accumulation but we have been unable to get this result consistently. Hence, we have not studied factors that mediate Fy localization.

\section{Gain of function phenotypes of PPE genes}

The expression of either in or fy driven by a variety of Gal4 drivers (e.g. ptc-Gal4, actGal4, ap-Gal4) did not result in a gain of function (gof) phenotype. This was surprising as the endogenous In (and Fy) protein accumulated at the proximal side of wing cells and when over expressed (oe) it was found throughout the cell (Fig. S1B-D) (Adler et al., 2004). Presumably, In (or Fy) is not active in that context. It is worth noting however that neither of these proteins accumulated to as high levels as we have seen for many other proteins in similar experiments. We found that co-over expressing in and fy together consistently resulted in a gain of function phenotype that consisted of cells that formed hairs that did not point distally and some of these cells formed multiple hairs (Fig. 1B-H). When ptc-GAL4 was used to drive expression this was typically manifested as a characteristic swirl in a relatively proximal region on the ventral 
surface of the wing (Fig. 1F). A multiple hair cell (mhc) phenotype and a milder polarity disruption were seen on the dorsal surface of such wings (Fig. 1B). Both the hair polarity and mhc phenotypes were enhanced by the addition of a second GAL4 expressing gene or a second UAS-in transgene (Fig. 1C,G,I, Appendix A). It was also enhanced when a second copy of both $U A S-H A$-in and $U A S-f y-G F P$ were present. As is described later, conditions that lead to a stronger hair phenotype also lead to a higher level of In protein accumulation. In contrast the addition of a second $U A S-f y-G F \mathrm{P}$ or $U A S-f y$-Flag-Ollas transgene partly suppressed the in/fy gof (Fig. 1D and H, Appendix A). Thus, while the oe of both genes is needed to generate a gain of function the In/Fy ratio is also important. We did not see clear evidence of a suppression of either phenotype when the flies were heterozygous for a loss of function mutation in the endogenous in gene or for the mhe phenotype when mutant for fy (Appendix A). We suggest that this is due to the level of oe being great enough that a $50 \%$ reduction in the endogenous protein was relatively insignificant.

Heterozygosity for a frtz mutation weakly but significantly suppressed the In/Fy gof and a low level of additional frtz supplied by a Ubi-myc-frtz-gfp transgene increased the strength of the phenotype but in this case the change was not significant (Appendix A). We also observed that heterozygosity for several of the PCP genes enhanced the in/fy gof mhc phenotype (Appendix A). pk, Vang also enhanced the polarity gof phenotype. Further, supplying a modest amount of extra Dsh partly suppressed both in/fy gof phenotypes (Table S1).

To determine if the in/fy gof polarity phenotype required the function of the $f z / s t a n$ pathway we examined flies that oe in and $f y$ while also being mutant for a third genes and determined if the characteristic in/fy swirl was still obvious. The characteristic swirl seen in ptc- 
Gal4 UAS-HA-in UAS-fy-GFP/ptc-Gal4 wings was not detected in ptc-Gal4 UAS-HA-in UAS$f y-G F P / p t c-G a l 4 ; f z^{P 21} / f z^{K 21}$ wings (Fig. $\left.1 \mathrm{I} \& \mathrm{~J}\right)$ and these latter wings were similar to the ptcGal4/+; $f z^{P 21} / f z^{K 21}$ wings (Fig. $1 \mathrm{~K}$ ). We concluded from this experiment that the function of the upstream $f z /$ stan genes was essential for generating the in/fy gof. We carried out similar experiments substituting $m w h$ for $f z$ and obtained equivalent results (Fig. 1L). That is, $m w h$ function was essential for the in/fy gof phenotype, implying that Mwh is essential for the transduction of the gof signal to the cytoskeleton.

In contrast to the results seen with in and $f y, f r t z$ over expression by itself resulted in a consistent gof (Fig. 2B). When untagged frtz was oe using ptc-gal4, the hairs pointed away from the midline of the wing; the location where ptc-G4 drives the highest level of expression (Fig. 2B). Hence, wing hairs appeared to be pointing from cells of higher toward cells of lower Frtz levels, much as they do when frizzled is over expressed in this way (Adler et al., 1997). The wing hair phenotype was qualitatively similar with two different $U A S$-frtz insertions (at attp2 and attp40) and dose sensitive. The ability of frtz oe to induce a PCP phenotype was also seen when alternate drivers were used. Ay-GAL4 flip out clones that drove expression of UAS-frtz resulted in a PCP gain of function and it was clear from stained pupal wings that frtz oe resulted in non-cell autonomous PCP abnormalities (Fig. 3E\&F). The hairs produced by neighboring cells pointed away from the clone, once again away from the higher frtz levels. Flip out clones examined in pupal wings during early stages of hair morphogenesis consistently showed delayed/slowed hair growth. In contrast to that seen for in and $f y$, the oe of frtz using some Gal4 drivers such as ap-Gal4 or act-Gal4 resulted in extensive lethality. In addition to the tissue polarity phenotype we also observed a distortion of the wing blade that led to a fold when frtz was oe with ptc-Gal4 (Fig S2). The frequency and severity of this fold was generally more 
dramatic in genotypes that gave stronger polarity phenotypes. In wings with a severe fold it was difficult to accurately assess the polarity phenotype. The frtz oe phenotype was sensitive to the dose of both other PPE genes and upstream PCP genes. Heterozygosity for in, $f y, f z$ and $p k$ resulted in an enhanced polarity phenotype (Fig S3A\&B) and heterozygosity for in, fy and $p k$ enhanced the number of mhc.

When expressed at a low level, frtz-GFP provided complete rescue of a null frtz allele (Yan et al., 2008b). Thus, our expectation was that oe Frtz-GFP would act like the oe Frtz, but that proved not to be the case. When frtz-GFP was expressed using ptc-gal4, hairs pointed toward the midline of the wing (Fig. 2A); the location where $p t c-G 4$ drives the highest level of expression. Thus hairs pointed from cells of lower toward cells of higher Frtz-GFP levels. This is also seen when PCP genes such as pk or stan are expressed in this pattern (Tree et al., 2002; Usui et al., 1999) and is the opposite of what we observed with the over expression of frtz. A qualitatively similar phenotype was seen with different $U A S$-frtz-GFP inserts. Flip out clones that expressed frtz-GFP displayed a non-cell autonomous PCP phenotype (Fig. 3C\&D) that was the opposite of that seen with frtz flip out clones. The hairs formed by neighboring cells pointed toward the clone, once again toward cells expressing higher levels of Frtz-GFP. We also observed a delay in hair formation in the Frtz-GFP over expression clones (Fig. 3A\&B) as well as increased cytoplasmic general F-actin staining. The delay and the increased cytoplasmic staining appeared cell autonomous. Thus, the oe of frtz and frtz-GFP produced a similar effect on hair growth despite their opposite effects on polarity. As was the case for frtz, the oe of frtzGFP using some Gal4 drivers such as actin-Gal4 resulted in extensive lethality. 
When Frtz-GFP was oe using ptc-Gal4 in flies that were simultaneously mutant for null (or strong) alleles of $f z, d s h$, in or $m w h$, the distinctive frtz-GFP polarity phenotype was lost (Fig. 2). Thus, the frtz-GFP gof phenotype is dependent on the activity of the $f z /$ stan pathway. It was also obvious that the resulting pattern was not a copy of the mutant $f z / s t a n$ pathway phenotype. Hence, when oe, frtz-GFP can also influence hair polarity by a mechanism that is independent of other components of the $f z /$ stan pathway.

\section{The over expression of PPE genes can re-pattern the accumulation of the PCP proteins}

The ability of oe of in+fy, frtz and frtz-GFP to produce abnormal wing hair polarity could be explained by either hair polarity becoming uncoupled from the accumulation of PCP proteins in these cells (as is the case in a PPE loss of function mutant) or by the over expression of the PPE genes resulting in re-patterning the accumulation of PCP proteins. The latter but not the former hypothesis can explain the non-autonomy seen in flip out clones that oe frtz or frtzGFP. To distinguish between these two hypotheses we examined the distribution of several PCP proteins (Fz, Stan, Vang, Dsh and Pk) in pupal wings that oe either frtz, frtz-GFP or in+fy. In all cases where the oe of a PPE protein produced gof hair polarity phenotype the zig zag accumulation pattern of PCP proteins was altered. In some cases there was a re-patterning so the high level edges were now rotated $90^{\circ}$ (e.g Fig. 4A'\& B') and in some cases there also appeared to be a change in the level of the PCP protein (e.g. Fig. 4C-H). For example the oe of Frtz lead to alterations in the zigzag accumulation of $\mathrm{Vang}$ and $\mathrm{Pk}$ (Fig. 4A'\&B') and an increase in the level of Dsh (Fig. 4C). However, as was seen for hair polarity, the oe of Frtz-GFP lead to the opposite effect, the decreased accumulation of Stan (Fig. 4G) and Dsh (Fig. 4H).

\section{The PPE genes form a physically interacting group}


The proximal accumulation of In (and Frtz) required the function of the other PPE genes (Adler et al., 2004; Strutt and Warrington, 2008). Based on such observations it seemed possible that at least In and Frtz were stabilized by binding to other PPE proteins. If that was the case we predicted that increased levels of Frtz would result in increased In levels and vice versa. We examined the accumulation of the endogenous In protein by immunostaining wings where either frtz-GFP, frtz or $f y-G F P$ were expressed using ptc-GAL4. We saw an increase in the level of endogenous In in cells that over expressed Frtz-GFP (Fig. 5A\&H) or Frtz (Fig. 5G\&H), which is consistent with the stabilization model. Consistent with the hair polarity phenotype seen with these genotypes the increased In was no longer found in a zigzag oriented along the anterior posterior axis. Rather, the accumulation of In was rotated $90^{\circ}$ (Fig. 5A). Thus, Frtz-GFP (and Frtz) not only stabilized In but also re-patterned its accumulation. Surprisingly, the expression of Fy-GFP caused a decrease in the accumulation of endogenous In (Fig. 5C\&H). This was a decrease and not a complete loss, consistent with the lack of effect of over expressing Fy on hair polarity. When both frtz and fy were over expressed In levels increased, thus higher Frtz levels were epistatic to higher Fy levels with respect to In accumulation (Fig. 5E\&H). We carried out similar experiments where we examined the effects of in and $f y$ over expression on the accumulation of Frtz expressed at a low level from a Ubi-my-frtz-GFP transgene. We used this transgene since we have not succeeded in generating an anti-Frtz antibody that can reliably detect the endogenous protein. We found that the over expression of in lead to increased accumulation of Frtz (Fig. 6A-D) while the over expression of fy lead to decreased accumulation ( Fig. 6E-H), although the results were not as dramatic as for In (Fig. 5).

To test if the ability of over expression of $f y$ or frtz to modulate the level of endogenous In could be functionally important we over expressed $f y$ or frtz in flies that carried a temperature 
sensitive hypomorphic allele of $i n$. Our hypothesis was that increased or decreased levels of the $\mathrm{In}^{\mathrm{ts}}$ protein would suppress or enhance the mutant phenotype. Consistent with this hypothesis we found that the over expression of $f y$-GFP enhanced the phenotype of $i n^{\text {ts }}$ (Fig. 7B\&C), while the over expression of $m y c-f r t z$ almost completely suppressed the phenotype (Fig. 7A\&B). Similar results were seen using both act-Gal4 and ptc-Gal4 to drive transgene expression.

We also carried out a similar set of experiments where we simultaneously over expressed two PPE proteins and determined by quantitative western blot analysis if one protein had an effect on the accumulation of the second. The simultaneous over expression of both in and $f r t z$ resulted in increased accumulation of both compared to single over expression controls (Fig. 6I,K,O). In contrast, when we simultaneously over expressed frtz and $f y$ the accumulation of both was decreased compared to single over expression controls (Fig. 6J,L,P). The situation was more complicated for the simultaneous over expression of in and fy (Fig. 6M,N,Q). The presence of a single $U A S$-fy gene resulted in increased accumulation of In, however the presence of two UAS-fy genes resulted in decreased accumulation of In. Thus the effects of Fy on In accumulation was dose sensitive. This dose sensitivity is similar to that seen for the in/fy gain of function hair polarity phenotype described earlier and provides an explanation for it.

As noted previously, when over expressed the proximal localization of In and Frtz was lost and the proteins were found throughout wing cells, although often enriched at the cell periphery. In co-immunolocalization experiments we found substantial co-localization of all three protein pairs (see Fig. S4). In other experiments we examined the localization of coexpressed proteins in the large salivary gland cells. We observed a clear co-localization of In 
and Fy in salivary gland cells. These were primarily at the cell periphery but in an irregular fashion. Similar results were seen for In and Frtz and Fy and Frtz (Fig. S4)

In several experiments we over expressed all 3 PPE genes. This resulted in the accumulation of the In, Fy and Frtz proteins in spherical puncta in pupal wing cells. The significance of these puncta is unclear. They were also seen in other cell types such as the salivary gland and third instar wing discs (Fig. S4).

The requirements for other PPE proteins for the proper accumulation of In and Frtz suggested the possibility that these proteins would interact physically in vivo. To test this hypothesis we determined if these proteins could be co-immunoprecipitated. In our initial experiments we used extracts of larvae that carried $h s-H A$-In and $h s-f y-F L A G$ transgenes $4 \mathrm{~h}$ after heat shock. We were able to detect In (using anti-HA) in immunoprecipitates of Fy (using anti-Flag) and Fy (using anti-Flag) in immunopreciptates of In (using anti-In) (Fig. 8A). In a second set of experiments we used Flag tagged Fy and wild type In and expressed both behind hsp70 promoters in $\mathrm{S} 2$ cells. We were able to detect In (using anti-In) in immunoprecipitates of Fy (using anti-Flag) (Fig. S5A) and in complementary experiments we could detect Fy (using anti-Flag) in immunopreciptates of In (using anti-In) (Fig. S6A). In another set of experiments we used UAS-HA-In and UAS-Fy-Flag-Ollas transgenes and wing discs and once again detected In in Ollas immunoprecipitates of Fy and Fy in immunoprecipitates of In (HA antibody) (Fig. $\mathrm{S5B})$.

Similar but less extensive experiments were done with Frtz and In. We found that antiHA antibodies could immunoprecipitate Frtz from ptc-Gal4 UAS-HA-in/+; UAS-myc-frtz/+ 
wing disc extracts (Fig. 8B). Similarly, we found that anti-Frtz antibodies could coimmunoprecipitate In from ptc-Gal4 UAS-HA-in/+; UAS-frtz-GFP/+ wing discs (Fig. S5C).

In contrast to the results noted above we did not see the co-immunoprecipitation of Fy and Frtz when we tested extracts from ptc-Gal4/UAS-fy-flag-ollas; UAS-myc-ftrz wing discs (anti-Frtz did not precipitate Fy-flag-ollas (Fig. 8C)).

Finally we found that anti-Fy antibodies could co-immunoprecipitate both In and Frtz from wing disc extracts from ptc-Gal4/UAS-HA-in UAS-fy-GFP; UAS-frtz-GFP/+ larvae (Fig. 8D) suggesting all 3 proteins are present in a common complex.

\section{In can interact directly with fy and frtz}

We tested the three PPE proteins for direct interactions using the yeast-two-hybrid system. We found that In could interact with both Fy and Frtz but we did not see any evidence for a direct interaction between Fy and Frtz (Fig. S6).

We also carried out a limited set of experiments to determine if the known proteinprotein interaction domains found in In and Frtz were essential for the interactions between PPE proteins. The Frtz protein contains a pair of WD40 repeats (aa 304-384) (Collier et al., 2005) in the amino terminal half of the protein as well as a predicted coiled coil region. There is also an atrophin 1 homology in the carboxyl terminal half of the protein (aa 644-879). In the yeast twohybrid system the amino terminal (aa 1-550) but not the carboxyl terminal region (aa 550-904) of Frtz could interact with In. We further split the amino terminal region into several parts (Fig. S7). The WD40 containing fragment interacted the most strongly, but we also obtained 
evidence for a weaker interaction between In and the coiled coil containing fragment of Frtz (Appendix A). Our data suggest there are two binding interactions between In and Frtz.

The In protein contains a PDZ domain (aa 158-213) (Cho et al., 1992; Te Velthuis et al., 2007) that is found in the amino terminal region (aa 1-550), while the C terminal half (aa 551869) contains no recognizable domains. We found that the $\mathrm{C}$ terminal domain of In could interact with both Frtz and Fy, while no interaction was seen with the amino terminal domain (Fig. S6). Thus, the PDZ domain does not appear to be important for the ability of In to interact with Frtz or Fy. 


\section{Discussion}

\section{PPE genes appear to be a functional and biochemical group}

A variety of genetic data suggested that the PPE genes in, fy and frtz comprised a functional group in Drosophila (Adler et al., 2004; Collier et al., 2005; Strutt and Warrington, 2008; Wong and Adler, 1993). Our data establishes that In binds directly to both Fy and Frtz. Our analysis of gain of function phenotypes suggested some functional differences between the genes, thus their physical interactions may not be obligate in some contexts, although caution is needed when interpreting gain of function phenotypes.

The vertebrate homologs of in, fy and frtz are known as intu, fuz and WDPCP (aka frtz). Mutations in these genes show both PCP and ciliogenesis phenotypes (Cui et al., 2013; Gray et al., 2009; Heydeck et al., 2009; Kim et al., 2010; Park et al., 2006; Zilber et al., 2013). In the mouse both intu and fuz mutations result in neural tube defects (Heydeck and Liu, 2011; Heydeck et al., 2009) and double mutants appear similar to single mutant (Heydeck and Liu, 2011) mirroring the lack of phenotypic additivity seen in flies (Collier et al., 2005). The similarity of these phenotypes is consistent with the interactions detected in the fly proteins being conserved in mammals. The ciliogenesis phenotype is not seen in flies where very few cells contain primary cilia. In humans frtz mutations have been implicated in Bardet-Biedl (BBS) syndrome (Kim et al., 2010) and fuz mutations have been implicated in neural tube defects (Seo et al., 2011). The defect in ciliogenesis results in defects in hedgehog signaling (Gray et al., 2009; Heydeck et al., 2009) and defects in Wnt and EGF signaling have also been reported in mutants (Tabler et al., 2013; Zhang et al., 2011; Zilber et al., 2013), although these could be secondary to the hedgehog effect. Defects in key signaling pathways that function in 
many locations and times in development complicate the interpretation of mutant phenotypes. A variety of data has suggested that intu and fuz might not function downstream of the PCP genes in some vertebrates and that the vertebrate homologs of the PPE genes might not share all of the same functions. A typical feature of PCP mutants in vertebrates is a convergent extension phenotype in early embryos (Goodrich and Strutt, 2011; Goto and Keller, 2002; Jessen et al., 2002; Wallingford et al., 2001) and this was also seen in intu and fuz morphants in Xenopus (Park et al., 2006). However, no evidence for such a convergent-extension phenotype was seen in intu and fuz mouse mutants (Heydeck and Liu, 2011). Further, a cochlea stereocilia phenotype seen in PCP mutants is present in frtz mutants (Cui et al., 2013) but has not been reported in intu and fuz mutants. The fuz gene has been found to be important for membrane trafficking (Gray et al., 2009) and retrograde intraflagellar transport (Brooks and Wallingford, 2012) and similar finding have not been reported for the other PPE genes.

Mutations in the Drosophila PPE genes produce notable planar polarity phenotypes in a subset of the tissues where such phenotypes are associated with mutations in the upstream PCP genes (Adler, 1992; Gubb and Garcia-Bellido, 1982). This could be due to the PPE genes only functioning in some cellular contexts or to the PPE proteins functioning downstream of the PCP genes in all tissues but not being important in some. For example, this could be due to redundancy with an alternative gene module in some cell types. In support of this latter hypothesis, when we examined the eye of in mutants we detected a very weak PCP phenotype (only a few ommatidia per eye were affected) that had been missed due to the lack of a rough eye phenotype (Lee and Adler, 2002). Some of the differences between the phenotypes of vertebrate PCP and PPE genes could have a similar basis. 
An interesting observation is that frtz mutant mice show a loss of Vangl2 in the cochlea (Cui et al., 2013). Thus, in the murine cochlea frtz is not "downstream" of Vangl2. Similarly, both intu and fuz regulate Dsh localization to the cilium (Park et al., 1996; Zilber et al., 2013). Thus, in these vertebrate tissues the PPE genes do not appear to be downstream of the PCP genes. These observations are reminiscent of our observations that the fly PPE genes are not obligatorily downstream of the PCP genes. Further, both intu and fuz being required for normal Dsh localization to the cilium suggests these two proteins functioning together in vertebrates.

In addition to the shared phenotypes frtz has also been found to interact with septin 7 and septin2 and to affect focal adhesions and cell migration (Cui et al., 2013; Kim et al., 2010). It was suggested that through its interaction with septins, frtz could regulate the actin cytoskeleton (Cui et al., 2013; Kim et al., 2010). Our observation that the over expression of Frtz leads to a substantial delay in hair initiation and to an altered F-actin distribution in wing cells prior to hair initiation also suggests an effect on the actin cytoskeleton independent of its role in PCP. Neither of these phenotypes were seen with in and fy. Thus, in both Drosophila and vertebrates Frtz appears to have activity that is not shared with In and Fy.

From co-immunoprecipitation experiments we obtained evidence that all three proteins are found in the same protein complex. However, in the conditions these experiments were carried out under all of the proteins accumulated in large puncta and this may reflect an abnormal complex. However, it is worth noting that when only two of the proteins are oe they co-localize in both wing and salivary gland cells in the absence of any large puncta and they can be co-immunoprecipitated. We and others have also found that when In and Frtz are expressed at normal or close to normal levels, they co-localize at the proximal edge of pupal wing cells 
(Adler et al., 2004; Strutt and Warrington, 2008). Strutt and colleagues also were able to show that Fy also localized at the proximal edge of wing cells (Strutt and Warrington, 2008). These observations all support the hypothesis that the 3 proteins are found in a common complex.

The role of Fy seems in some ways different from that of In and Frtz. At least its interaction with the other two PPE proteins stands out. In the absence of $f y$ function In accumulation is sharply decreased however when oe Fy also decreases the accumulation of

endogenous In. Further when In and Fy are both oe the level of In accumulation can be increased or decreased depending on the relative dose of Fy. Thus, the stoichiometry of the two proteins appears to be the key. The importance of the relative In/Fy dose may also explain the requirement for the oe of both to produce a gof wing hair polarity phenotype. The relative ratio of Frtz and Fy also appears to be important as oe of Fy leads to decreased accumulation of Frtz encoded by either the ubiquitin promoter driven gene or by the UAS/Gal4 system. Nothing is currently known about the stoichiometry of a PPE protein complex and the number of types of such complexes. Such information might provide an explanation for the complex relationship between Fy and In (and Frtz). In vertebrates Fuz functions in intraflagellar transport, membrane vesicle fusion and secretion (Brooks and Wallingford, 2012; Gray et al., 2009). Perhaps in flies it functions in the intracellular trafficking of In and Frtz.

\section{PPE and PCP proteins}

Our experiments established that the oe of either In+Fy or Frtz by itself could alter the level and/or subcellular location where PCP proteins accumulated. These results were surprising as the PPE genes are generally thought to function downstream of the PCP proteins. As noted earlier in the mouse a frtz mutation altered Vangl2 accumulation in hair cells (Cui et al., 2013) 
and Intu and Fuz mutations altered Dsh recruitment to the cilium (Park et al., 2006; Zilber et al., 2013). Those and our results have blurred the hierarchy between these two groups of genes.

The formation of the proximal and distal protein domains in wing cells is thought to involve both inter and intra cellular signaling. It is generally thought that negative interactions between PCP proteins that accumulate on the proximal and distal edges of wing cells are part of an intracellular negative feedback loop that helps establish and maintain the localized protein domains (Amonlirdviman et al., 2005; Bastock et al., 2003; Das et al., 2004; Tree et al., 2002). One way in which the oe of the cytoplasmic PPE proteins might re-pattern the upstream proteins would be to interfere with this negative feedback loop. For example, the binding of a PPE protein to a PCP protein such as Pk might block $\mathrm{Pk}^{\prime} \mathrm{s}$ antagonistic interaction with Dsh. Alternatively, PPE proteins when oe could bind to and stabilize one or more of the PCP proteins (e.g. Pk or Dsh). For example, such an interaction might prevent the degradation of the PCP protein. Ubiquitin dependent $\mathrm{Pk}$ degradation has been implicated in regulating PCP (Narimatsu et al., 2009) and increased protein levels could result in the hair polarity phenotypes as the oe of any of the PCP proteins leads to alterations in wing hair polarity (Adler et al., 1997; Tree et al., 2002; Usui et al., 1999). There is also good precedent for the over expression of one PCP protein altering the levels of others. For example, it is known that the oe of $\mathrm{Pk}$ leads to increased accumulation of Fz, Dsh and Stan (Tree et al., 2002). Further studies will be needed to elucidate the mechanism by which the PPE proteins can influence the upstream proteins.

\section{Frtz and the cytoskeleton}

Our experiments on frtz also provided several novel and surprising results. The oe of $f r t z$ and frtz-GFP led to complementary effects on hair polarity. This was unexpected since when 
expressed at close to normal levels both proteins provide complete rescue of null frtz alleles. The basis for the complementary gof phenotypes remains unclear. Perhaps the folding of the GFP tagged protein is slowed and when over expressed much of it ends up in an abnormal conformation with abnormal activity with respect to PCP. Despite the complementary polarity phenotypes we detected a similar dramatic delay in hair outgrowth in flip-out clones of both. This suggested the possibility that at least when over expressed Frtz could inhibit the activation of the actin cytoskeleton. A second set of observations consistent with an effect on the actin cytoskeleton are bristle morphology abnormalities associated with over expression of frtz (or frtz-GFP) (pna, unpublished). Some effects on hair and bristle morphogenesis can be seen with other PCP and PPE genes but none as dramatic as those we have seen for frtz. Driving expression of frtz by ptc-Gal4 also led to a fold in the wing that runs down the ptc domain. The basis for this is unclear but could be due to changes in cell size, cell number or shape. We have not detected similar phenotypes in either gain or loss of function experiments with other $f z /$ stan pathway mutants. These observations suggest that Frtz may modulate the cytoskeleton independently of the $f z /$ stan pathway as a whole.

Several other observations suggest that Frtz plays multiple roles during development and that at least one in planar cell polarity is likely to be independent of the $f z / s t a n$ pathway. In ptc $>$ frtz-GFP wings a very distinctive hair polarity phenotype was seen. When wings were $p t c>f r t z-G F P$ and also mutant for another $f z /$ stan pathway gene this distinctive polarity pattern was lost. However, the hair polarity pattern also did not closely resemble that of the fz/stan pathway mutant. This indicates that $f r t z-G F P$ can influence hair polarity in a $f z / s t a n$ pathway independent manner. The mechanism and the identity of a presumptive second pathway remain to be elucidated. One possibility is that frtz might function as an effector of the dachsous/fat 
pathway in a $f z / s t a n$ independent manner. A second is that Frtz might regulate the actin cytoskeleton (Cui et al., 2013; Kim et al., 2010) independently of its role in the $f z /$ stan pathway. 
Figure 1. The in/fy gain of function wing phenotype. All images are from the $\mathrm{C}$ region of the wing (between the 3rd and 4th veins just anterior to the posterior cross vein). This region is in the ptc domain. (A-D) shows the dorsal surface of the wing and (E-L) the ventral surface. The genotypes are noted in each panel. Ore-R shows the wild type hair pattern. 


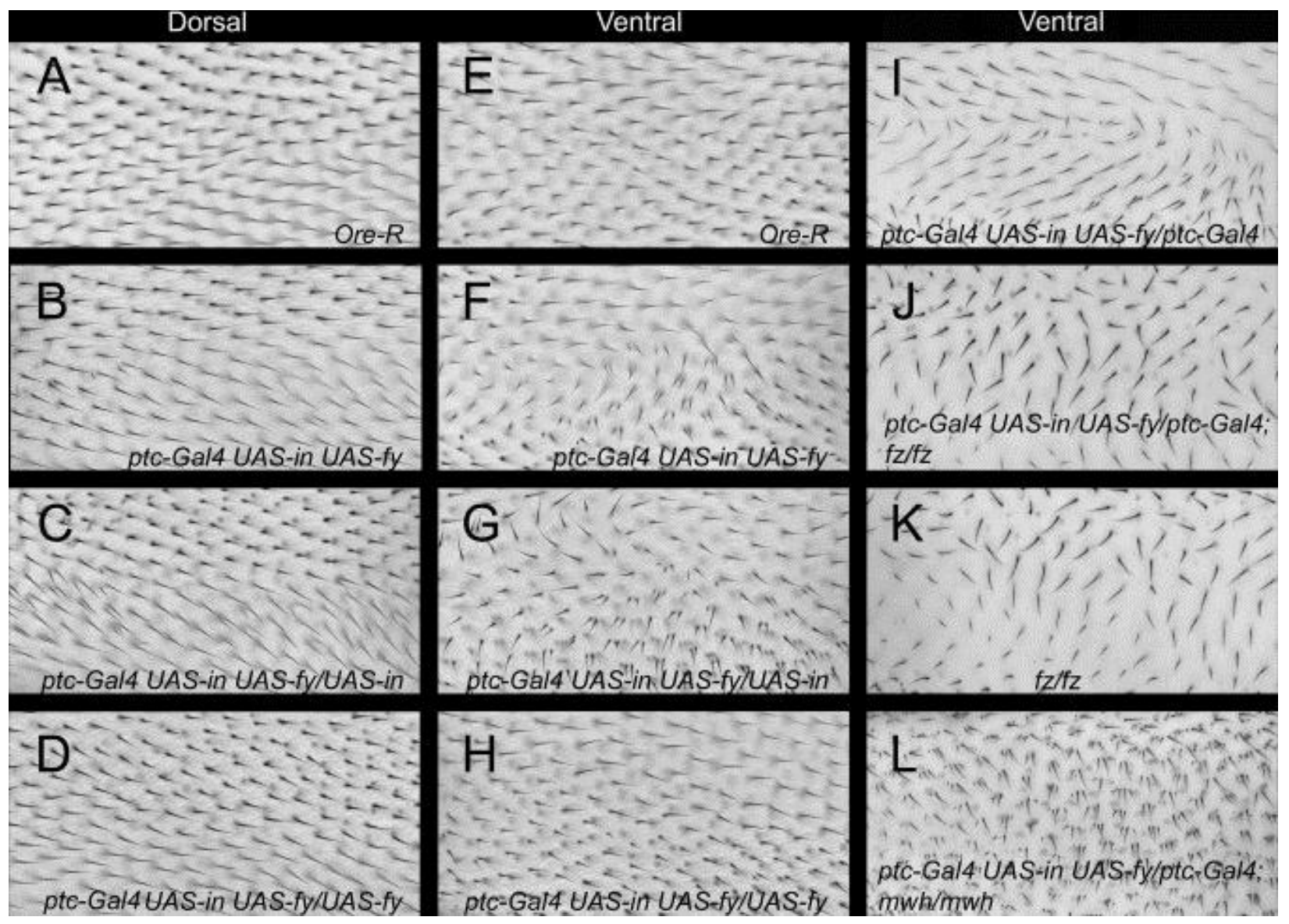


Figure 2. The over expression of frtz and frtz-GFP result in opposite gain of function PCP phenotypes. All images are from the $\mathrm{C}$ region on the dorsal surface of the wing (between the 3rd and 4th veins just anterior to the posterior cross vein). This region is in the ptc domain. The genotypes are noted in each panel. 


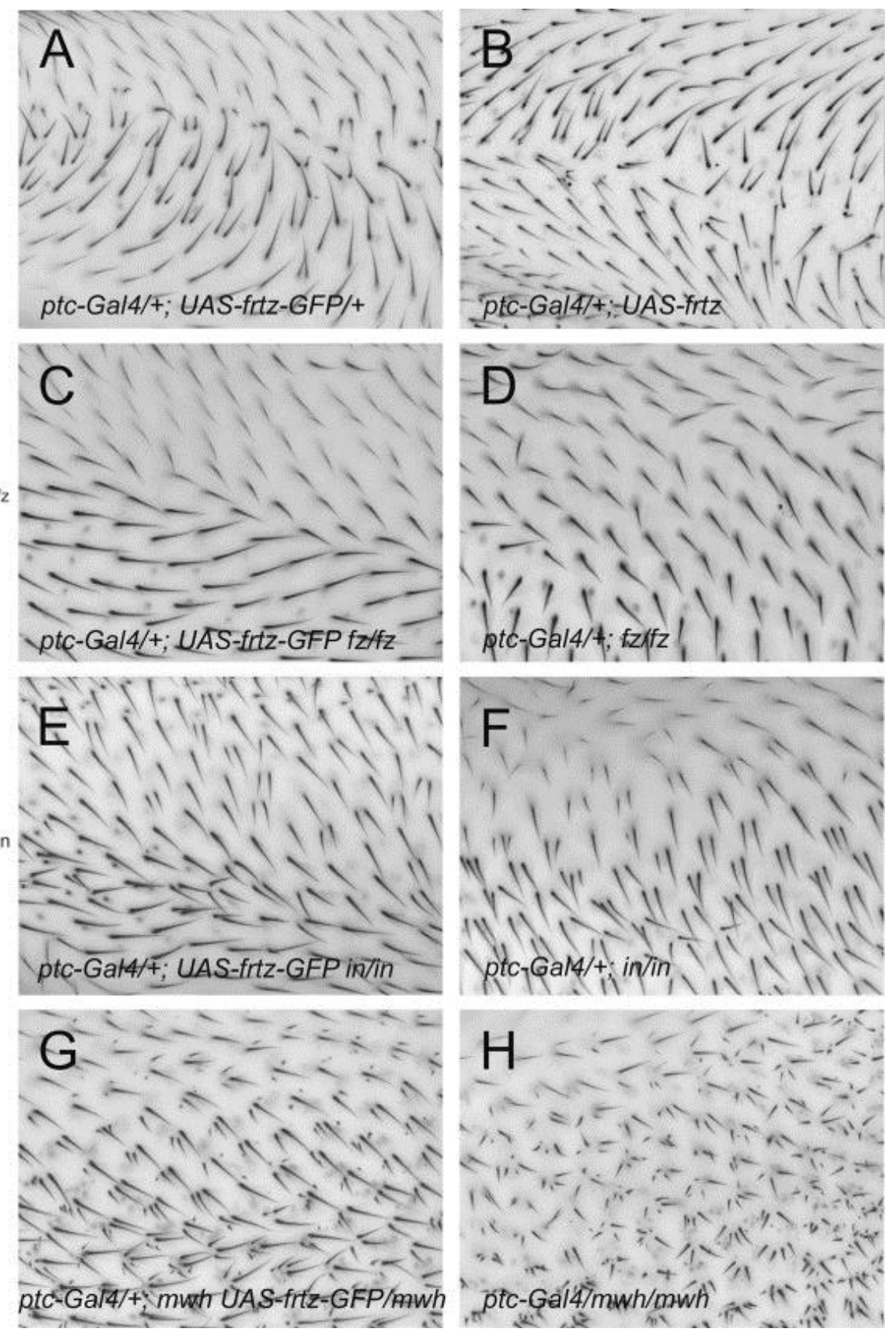


Figure 3.The gain of function PCP phenotypes associated with over expression of frtz and frtzGFP can affect the polarity of neighboring wing cells. (A) and (B) show a flip out clone that expresses Frtz-GFP. This results in a cell autonomous delay in hair formation. The asterisk marks the region showing hair delay. (C) and (D) show a clone in an older wing. The asterisk marks a region of wild type cells distal to the clone that forms hairs that point toward the clone. (E) and (F) show a flip out clone that over expresses Frtz. Note the delay in hair formation of clone cells. The asterisk marks a region of wild type cells proximal to the clone that forms hairs that point away from the clone. 


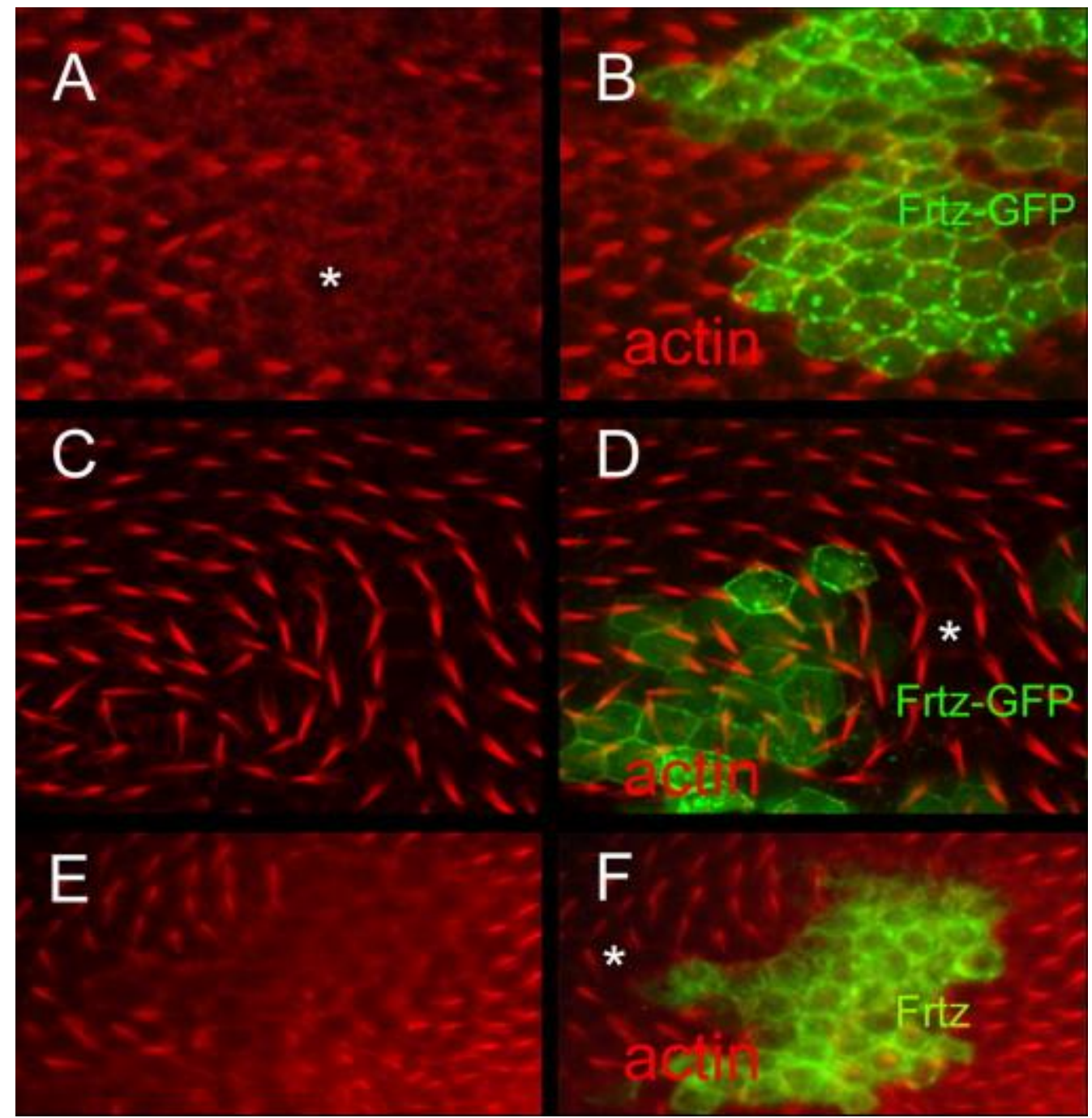


Figure 4. The over expression of PPE genes can alter the accumulation of PCP proteins. (A) and $\left(\mathrm{A}^{\prime}\right)$ are from the same ptc-Gal4/+; UAS-frtz/act-Vang-YFP wing. (A) is from outside the ptc domain and $\left(\mathrm{A}^{\prime}\right)$ from inside the domain. Vang-YFP immunostaining is shown. Outside the ptc domain Vang accumulates in the typical proximal/distal zig-zag pattern. Inside the ptc domain Vang accumulation is more punctate and is preferentially found on the anterior/posterior sides of cells. $\mathrm{B}$ and $\left(\mathrm{B}^{\prime}\right)$ are from the same ptc-Gal4/+; UAS-frtz wing. (B) is from outside the ptc domain and $\left(\mathrm{B}^{\prime}\right)$ from inside the domain. Endogenous $\mathrm{Pk}$ accumulation is shown by immunostaining. Outside the ptc domain $\mathrm{Pk}$ accumulates in the typical proximal/distal zig-zag pattern. Inside the ptc domain $\mathrm{Pk}$ accumulation is more punctate and is preferentially found on the anterior/posterior sides of cells. (C) shows a ptc-Gal4/dsh-myc; UAS-frtz/+ wing that was immunostained with anti-Myc antibody to show the accumulation of Dsh-myc. (E) shows a ptcGal4 UAS-HA-in UAS-fy-GFP/+ wing immunostained to show the accumulation of the endogenous $\mathrm{Pk}$ protein. Note the increased $\mathrm{Pk}$ accumulation and altered zigzag accumulation. (E) shows a ptc-Gal4 UAS-HA-in UAS-fy-GFP/+ wing immunostained to show the accumulation of the endogenous Stan protein. Note the loss of the distinct proximal/distal zigzag in the ptc domain. (F) shows a ptc-Gal4/+; UAS-frtz-GFP/+ wing immunostained to show the accumulation of the endogenous $\mathrm{Pk}$ protein. Note the slightly increased accumulation of $\mathrm{Pk}$ and the loss of the proximal/distal zigzag in the ptc domain. 

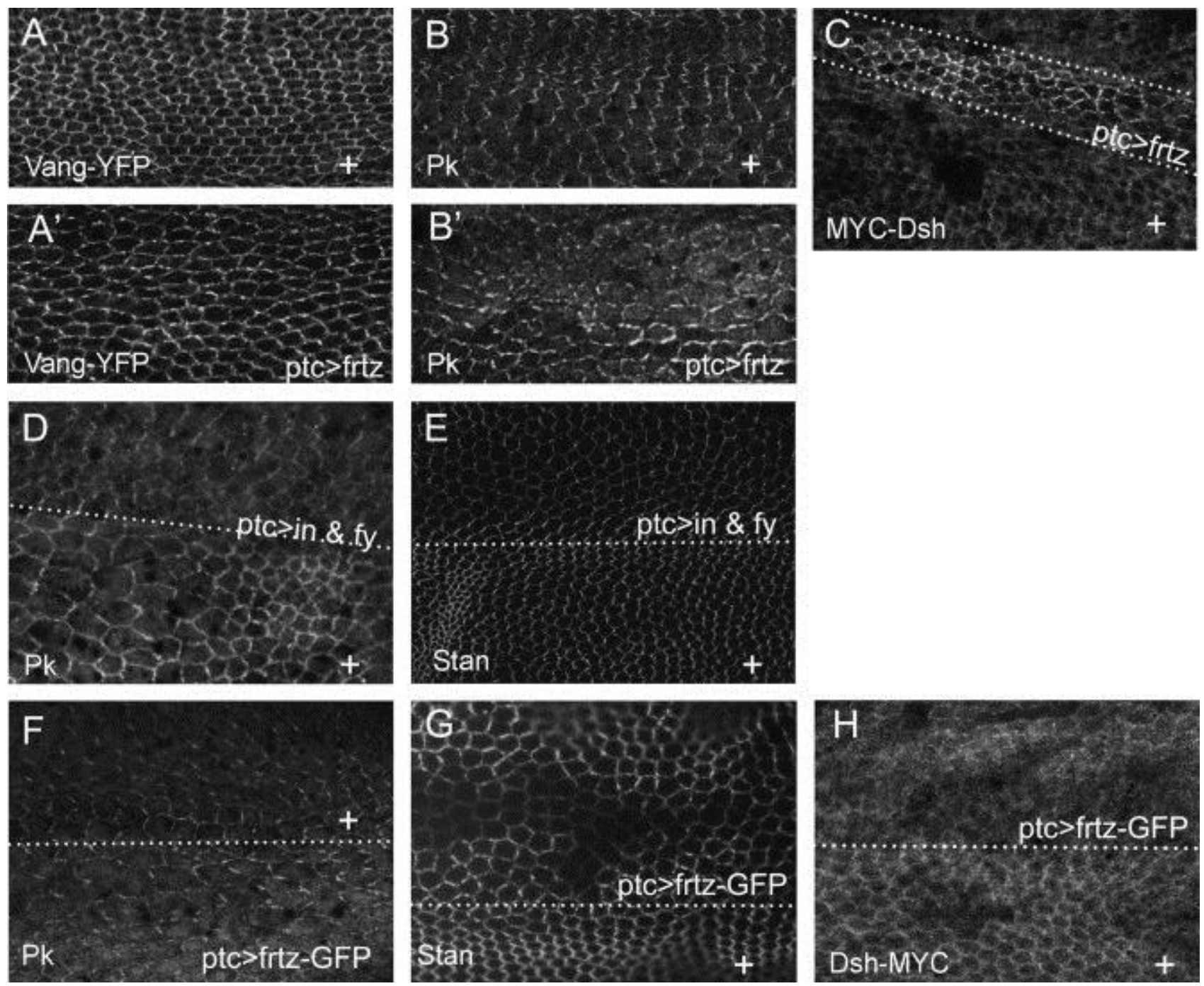
Figure 5. In accumulation is altered in wing cells by the over expression of $f r t z$ and $f y$. (A) and (B) show a ptc-Gal4/+; UAS-frtz-GFP/+ wing immunostained to show the endogenous In protein. The arrow marks the approximate location of the edge of the ptc-domain. Note the increased accumulation of In and its altered accumulation pattern associated with the over expression of frtz-GFP. (C) and (D) show a ptc-Gal4/+; UAS-fy-GFP/+ wing immunostained to show the endogenous In protein. The arrow marks the approximate location of the edge of the ptc-domain. Note the decreased accumulation of In associated with the over expression of $f y$ GFP. (E) and (F) show a ptc-Gal4/UAS-fy-GFP; UAS-frtz-GFP/+ wing immunostained to show the endogenous In protein. The arrow marks the approximate location of the edge of the ptcdomain. Note the increased accumulation of In and its altered accumulation pattern associated with the over expression of frtz-GFP. (G) shows a ptc-Gal4/UAS-frtz wing immunostained to show the endogenous In protein. The arrow marks the approximate location of the edge of the ptc-domain. Note the increased accumulation of In where frtz was overexpressed. $(\mathrm{H})$ shows the quantitation of the changes in In immunostaining inside and outside of the ptc domain. Average grey scale values in the ptc domain are normalized to the level outside of the ptc domain. A $t$ test was used to compare the grey values inside and outside of the ptc domain. ${ }^{*} p=0.05-0.01$, *** $p=0.01-0.001, \stackrel{* * *}{p} p<0.001$. 

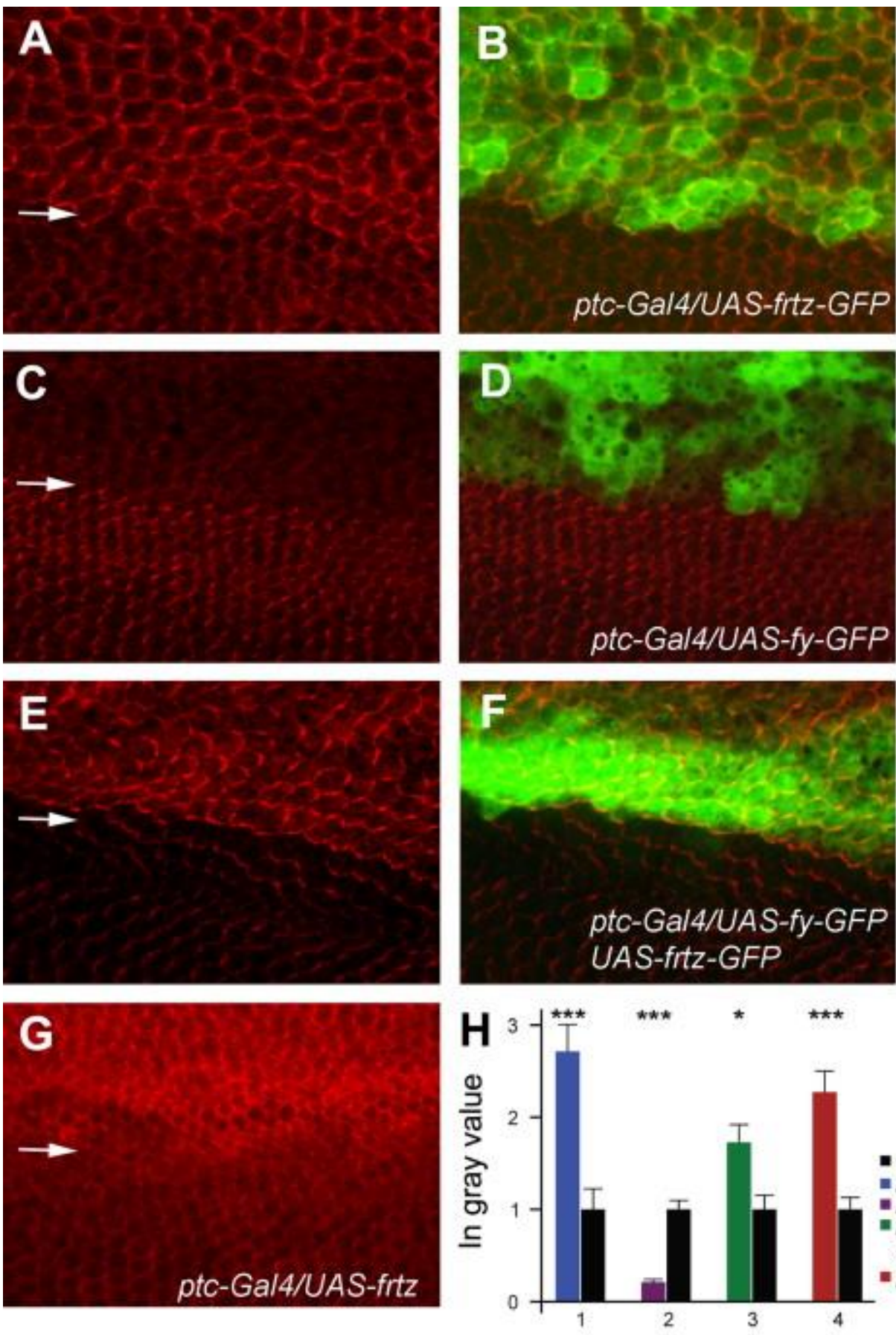

- outside of ptc domain - ptc-Gal4/UAS-fitz-GFP

- ptc-Ga/4/UAS-fy-GFP

- ptc-Ga/4/UAS-fy-GFP.

UAS-fitz-GFP/+

n ptc-Ga/4/UAS-fitz 
Figure 6. The over expression of one PPE protein affects the accumulation of the others. (A), (B) and (C) show a ptc-Gal4/UAS-HA-in; ubi-myc-frtz-GFP/+ wing immunostained for both In (red) and GFP (green-Frtz). This $u b i-m y c-f r t z-G F P$ transgene gives variegated expression. D shows the quantitation of GFP (Frtz) staining inside (2) and outside (1) of the ptc domain. (Note the quantitation was done on the peripheral accumulation in cells that expressed high levels of Frtz). The increase in Frtz accumulation was significant $(p<0.05)$. (E), (F) and $(\mathrm{G})$ shows a ptcGal4/UAS-fy-Flag-ollas; ubi-myc-frtz-GFP/+ wing immunostained for both Ollas (red-Fy) and GFP (green-Frtz). H shows the quantitation of GFP (Frtz) staining inside (2) and outside (1) of the ptc domain. The decrease in Frtz accumulation was significant $(p<0.05)$. (I), (J) and (K) show the results of Western blots of wing disc samples where the expression of UAS-HA-in and $U A S-m y c-f r t z$ were driven either singly or together using ptc-Gal4. (I) and (J) show the quantitation and $\mathrm{K}$ the blot. $(\mathrm{M}),(\mathrm{N})$ and $(\mathrm{L})$ show the results of Western blots of wing disc samples where the expression of UAS-fy-Flag-Ollas and UAS-myc-frtz were driven either singly or together using ptc-Gal4. (L) and (M) show the quantitation and (N) the blot. (O), (P) and (Q) show the results of Western blots of wing disc samples where the expression of UAS-HA-in and $U A S-f y-G F P$ were driven either singly or together in varying doses using ptc-Gal4. (O) and $\mathrm{P}$ show the quantitation and (Q) the blot. 

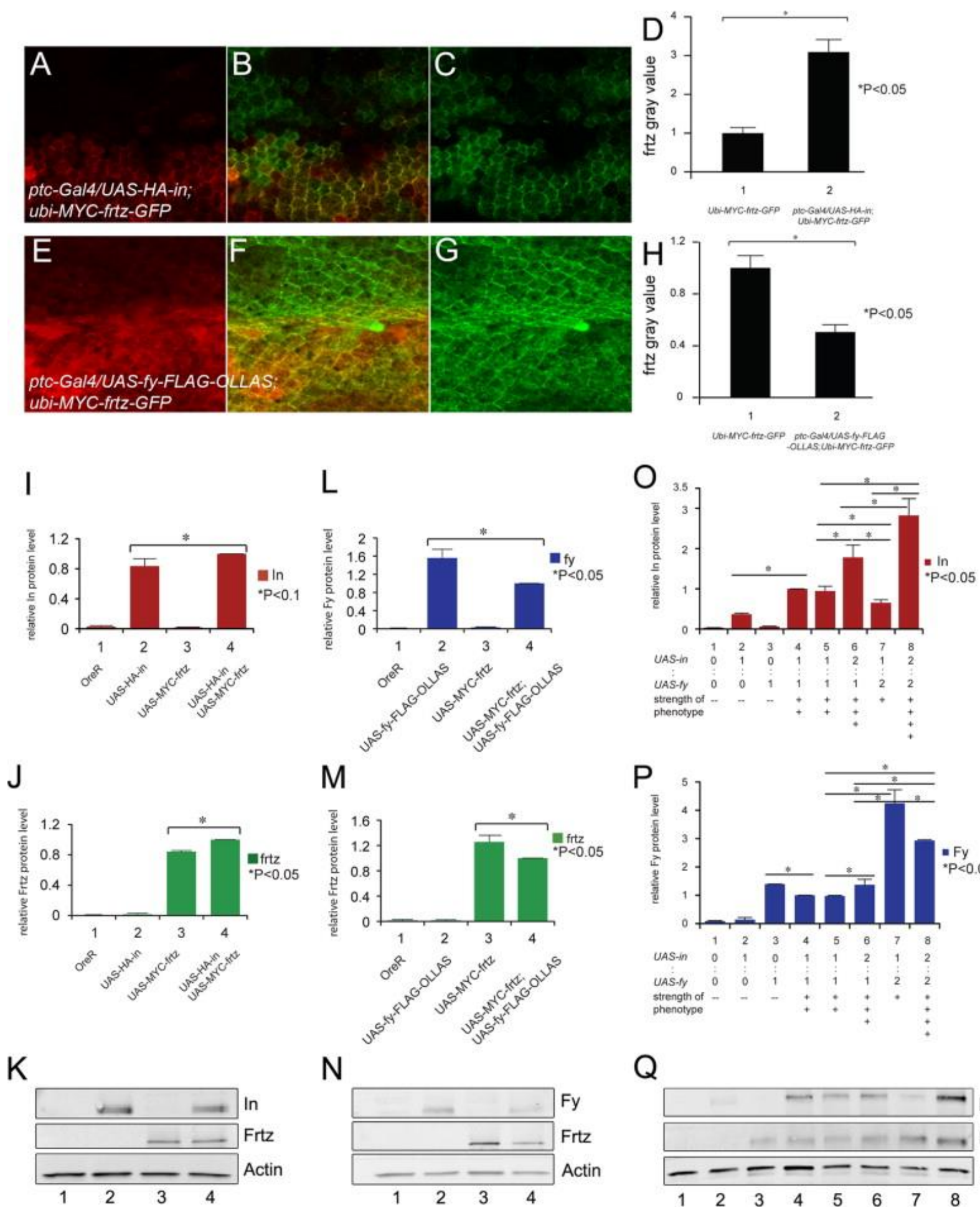

$\mathrm{N}$

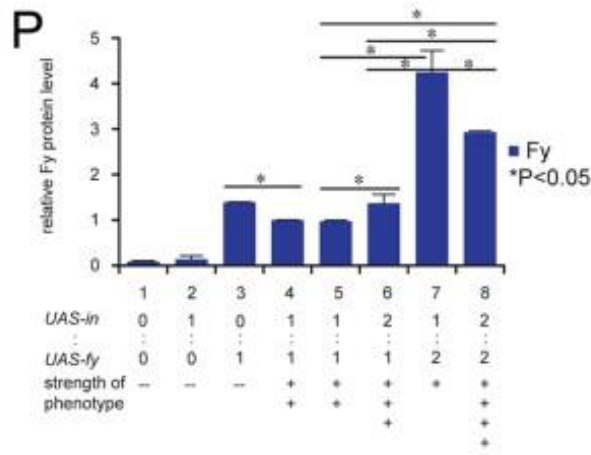

Q
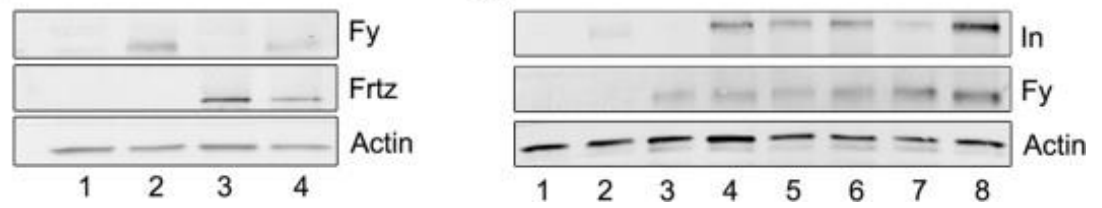
Figure 7. The over expression of $f y$ and $f r t z$ modifies a hypomorphic in phenotype. Panel B is a control showing the $i n^{t s}$ phenotype at $25^{\circ} \mathrm{C}$. The wings in (A) and (C) used actin-Gal4 to drive the expression of $m y c-f r t z$ and $f y-G F P$ respectively. 

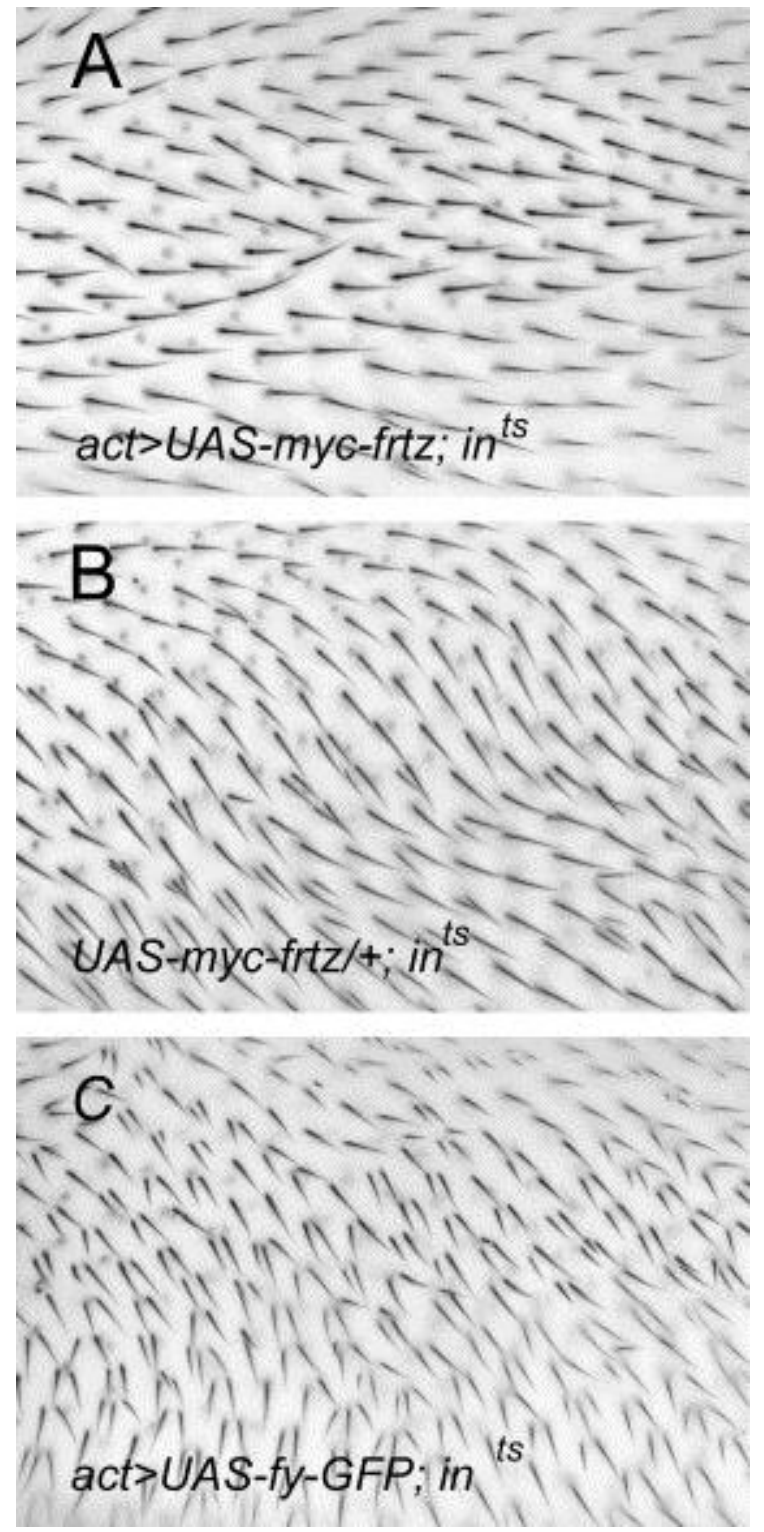
Figure 8. Co-immunoprecipitation of PPE proteins. (A) shows an experiment where HA-In and Fy-Flag were co-immunoprecipitated by either anti-In or anti-Flag antibodies. In this experiment a heat shock was used to induce the expression of transgenes sub-cloned behind the hsp70 promoter. (B) shows the co-immunoprecipitation of In and Frtz. This experiment used $U A S-H A$-in and UAS-myc-frtz transgenes driven by ptc-Gal4. Wing disc samples were immunoprecipitated using Rabbit anti-HA antibody. Arrows point to myc-Frtz and HA-In on the Western blots. (C) shows an experiment where we tested the co-immunoprecipitation of Fy and Frtz. Samples from ptc-Gal4/UAS-fy-Flag-Ollas; UAS-myc-frtz/+ wing discs were IP with anti-Myc antibody and then assayed by western blotting. Lane 3 and 4 (on the left side) are duplicates. The arrows point to Fy-Flag-Ollas (left) and myc-Frtz (right). Note that Fy was not precipitated with Frtz. (D) shows an experiment where all three of the PPE proteins were coexpressed (ptc-Gal4/UAS-HA-in UAS-fy-GFP; UAS-frtz-GFP/+). Samples were made from wing discs and immunoprecipitated using anti-Fy antibody. The Western blots were probed with anti-GFP (to detect both Fy-GFP and Frtz-GFP) and anti-In. Control experiments established that anti-Fy antibody could not pull down either HA-in or Frtz-GFP (data not shown). 
A

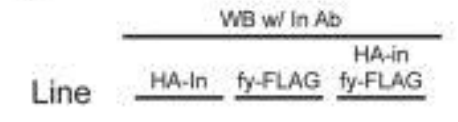

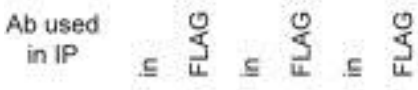

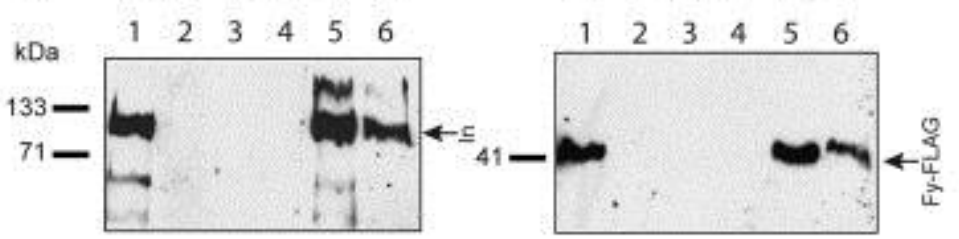

C

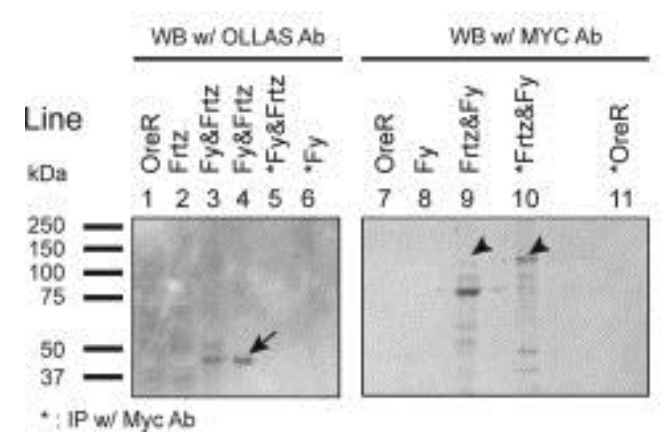

B

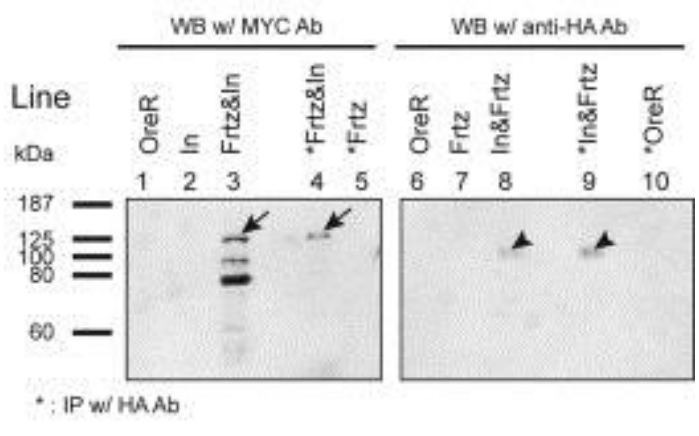

D

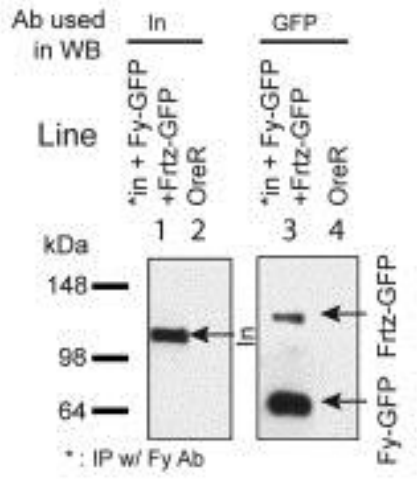


Table \$1

Genetic enhancement/suppression of the in fy polarity phenotype.

\begin{tabular}{|c|c|c|c|c|c|c|c|c|}
\hline Gal4 & UAS-in & UAS-fy & other & \begin{tabular}{|l}
$\#$ \\
wings \\
\end{tabular} & $\begin{array}{l}\text { Mean polarity Score } \\
\text { Ventral^ (sd) }\end{array}$ & $\mathrm{P}^{*}=$ & $\begin{array}{l}\text { Mean polarity Score } \\
\text { Dorsal" }^{*} \text { (sd) }\end{array}$ & $\mathrm{P}^{*}=$ \\
\hline ptc $-1^{8}$ & HA-in-1 & $f y-G F P-1$ & & 68 & $2.98(0.12)$ & NR & $2.06(0.29)$ & NR \\
\hline$p t c-2$ & HA-in-1 & $f y-G F P-1$ & & 7 & $3(0)$ & 0.95 & $2.57(0.53)$ & $<0.001$ \\
\hline ptc - 1, Tub-1 & HA-in-1 & fy-GFP -1 & & 15 & $3(0)$ & 0.94 & $2.67(0.49)$ & $<0.001$ \\
\hline ptc -1 & HA-in-2 & $f y-G F P-1$ & & 39 & $3(0)$ & 0.90 & $2.87(0.34)$ & $<0.001$ \\
\hline ptc -1 & HA-in-2 & $f y-G F P-2$ & & 22 & $3(0)$ & 0.92 & $3(0)$ & $<0.001$ \\
\hline$p t c-1$ & HA-in-2 & $\begin{array}{l}\text { fy-GFP - } 1, f y- \\
\text { flag-ollas-1 }\end{array}$ & & 20 & $3(0)$ & 0.92 & $2.70(0.47)$ & $<0.001$ \\
\hline ptc -1 & HA-in-1 & $f y-G F P-2$ & & 20 & $2(0.45)$ & $<0.001$ & $1.58(0.51)$ & 0.003 \\
\hline ptc -1 & HA-in-1 & $\begin{array}{l}\text { fy-GFP -1, fy- } \\
\text { flag-ollas } \\
\end{array}$ & & 33 & $2.52(0.66)$ & 0.002 & $1.66(0.55)$ & 0.003 \\
\hline ptc -1 & HA-in-1 & & & 10 & $1(0)$ & $<0.001$ & $1.0(0)$ & $<0.001$ \\
\hline ptc - 1 & & fy-GFP -1 & & 15 & $1(0)$ & $<0.001$ & $1.0(0)$ & $<0.001$ \\
\hline ptc -1 & HA-in-1 & $f y-G F P-1$ & $p k-1 /+$ & 20 & $3(0)$ & 0.92 & $2.95(0.22)$ & $<0.001$ \\
\hline ptc -1 & HA-in-1 & $f y-G F P-1$ & $p k^{p k-\text { sple } 13} /+$ & 13 & $3(0)$ & 0.94 & $3(0)$ & $<0.001$ \\
\hline ptc -1 & HA-in-1 & $f y-G F P-1$ & $\operatorname{Vang}^{25} /+$ & 15 & $2.93(0.26)$ & 0.76 & $2.93(0.26)$ & $<0.001$ \\
\hline ptc -1 & HA-in-1 & fy-GFP -1 & $f z^{p 21} /+$ & 28 & $2.93(0.26)$ & 0.67 & $2.18(0.40)$ & 0.35 \\
\hline ptc -1 & HA-in-1 & $f y-G F P-1$ & $f z^{k 21} /+$ & 19 & $2.75(0.44)$ & 0.11 & $2(0)$ & 0.70 \\
\hline ptc -1 & HA-in-1 & $f y-G F P-1$ & $\operatorname{stan}^{3-71} /+$ & 23 & $2.91(0.29)$ & 0.61 & $2.05(0.21)$ & 0.93 \\
\hline ptc -1 & HA-in-1 & $f y-G F P-1$ & $d s h^{1} /+$ & 16 & $2.88(0.34)$ & 0.50 & $2(0)$ & 0.662 \\
\hline$p t c-1$ & HA-in-1 & $f y-G F P-1$ & $f y^{1} /+$ & 36 & $2.43(0.50)$ & $<0.001$ & $1.86(0.55)$ & 0.123 \\
\hline ptc -1 & HA-in-1 & $f y-G F P-1$ & frtz $z^{3521} /+$ & 44 & $2.43(0.50)$ & $<0.001$ & $1.74(0.44)$ & 0.009 \\
\hline ptc -1 & \begin{tabular}{|l|} 
HA-in-1 \\
\end{tabular} & $f y-G F P-1$ & in $^{1} /+$ & 26 & $2.92(0.60)$ & 0.49 & \begin{tabular}{|l|}
$2(0.47)$ \\
\end{tabular} & 0.69 \\
\hline ptc -1 & HA-in-1 & fy-GFP -1 & arm-fz-GFP/+ & 13 & $3(0)$ & 0.94 & $1.84(0.38)$ & 0.25 \\
\hline ptc -1 & HA-in-1 & $f y-G F P-1$ & $\begin{array}{l}\text { Cas-dsh- } \\
\text { GFP } /+\end{array}$ & 23 & $2.91(0.29)$ & 0.61 & $1.46(0.59)$ & $<0.001$ \\
\hline ptc -1 & HA-in-1 & $f y-G F P-1$ & $\begin{array}{l}\text { Ubi-myc-frtz- } \\
\text { GFP }\end{array}$ & 39 & $2.98(0.72)$ & 0.93 & $2.44(0.53)$ & 0.066 \\
\hline
\end{tabular}

$\&=$ number of copies, ${ }^{*}$ Mann-Whitney rank sum test, ${ }^{\wedge} 1=0-30^{\circ}, 2=30^{\circ}-90^{\circ}, 3>90^{\circ}, \# 1=0-30^{\circ}, 2=30^{\circ}-60^{\circ}, 3>60^{\circ}$ 
Table $\$ 2$

Genetic enhancement/suppression of the in fy multiple hair cell phenotype.

\begin{tabular}{|c|c|c|c|c|c|c|}
\hline Gal4 & UAS-in & UAS-fy & other & Mean \# mhc & $\begin{array}{l}\text { Number of } \\
\text { wings }\end{array}$ & $P^{\varepsilon}=$ \\
\hline ptc $-1^{*}$ & HA-in-1 & $f y-G F P-1$ & & $4.16(3.0)$ & 55 & NR \\
\hline ptc -2 & HA-in-1 & $f y-G F P-1$ & & $18.33(8.2)$ & 6 & $<0.001$ \\
\hline ptc $-1, T u b-1$ & HA-in-1 & $f y-G F P-1$ & & $25.60(7.3)$ & 15 & $<0.001$ \\
\hline ptc -1 & HA-in-2 & $f y-G F P-1$ & & $42.51(18.2)$ & 37 & $<0.001$ \\
\hline ptc -1 & HA-in-2 & $f y-G F P-2$ & & $72.18(12.5)$ & 22 & $<0.001$ \\
\hline$p t c-1$ & HA-in-2 & $\begin{array}{l}f y \text {-GFP - } 1 \text {, } \\
\text { fy-filog-ollas- } \\
1\end{array}$ & & $25.2(5.7)$ & 19 & $<0.001$ \\
\hline ptc -1 & HA-in-1 & $f y-G F P-2$ & & $2.80(2.2)$ & 15 & $=0.10$ \\
\hline ptc -1 & HA-in-1 & $\begin{array}{l}f y \text {-GFP - } 1, \\
\text { fy-fiag-ollas }\end{array}$ & & $0.32(0.55)$ & 28 & $<0.001$ \\
\hline ptc -1 & HA-in-1 & & & $0(0)$ & 10 & $<0.001$ \\
\hline ptc -1 & & $f y-G F P-1$ & & $0(0)$ & 15 & $<0.001$ \\
\hline$p t c-1$ & HA-in-1 & $f y-G F P-1$ & $p k-1 /+$ & $53.71(21.6)$ & 14 & $<0.001$ \\
\hline ptc -1 & HA-in-1 & $f y-G F P-1$ & $p k^{p \operatorname{lik} s l e} /+$ & $7.60(4.6)$ & 15 & $=0.01$ \\
\hline ptc -1 & HA-in-1 & $f y-G F P-1$ & $\operatorname{Vang}^{25} /+$ & $29.55(17.1)$ & 11 & $<0.001$ \\
\hline ptc -1 & HA-in-1 & $f y-G F P-1$ & $f z^{p 21} /+$ & $11.24(5.9)$ & 38 & $<0.001$ \\
\hline ptc -1 & HA-in-1 & $f y-G F P-1$ & $f z^{K 21} /+$ & $17.59(7.94)$ & 17 & $<0.001$ \\
\hline ptc -1 & HA-in-1 & $f y-G F P-1$ & $\operatorname{stan}^{3-71} /+$ & $12.29(4.6)$ & 24 & $<0.001$ \\
\hline ptc -1 & HA-in-1 & $f y-G F P-1$ & $d s h^{1} /+$ & $11.13(7.4)$ & 15 & $<0.001$ \\
\hline ptc -1 & HA-in-1 & $f y-G F P-1$ & $f y^{1} /+$ & $4.00(2.1)$ & 22 & $=0.92$ \\
\hline ptc -1 & HA-in-1 & $f y-G F P-1$ & $f y^{2} /+$ & $4.17(3.6)$ & 18 & $=0.81$ \\
\hline ptc -1 & HA-in-1 & $f y-G F P-1$ & $\mathrm{frtz}^{3521} /+$ & $2.65(2.2)$ & 48 & $=0.01$ \\
\hline ptc -1 & HA-in-1 & $f y-G F P-1$ & $\mathrm{in}^{1} /+$ & $4.11(3.3)$ & 27 & $=0.79$ \\
\hline ptc -1 & HA-in-1 & $f y-G F P-1$ & $\begin{array}{l}\text { arm-fz- } \\
\text { GFP/+ }\end{array}$ & $5.76(4.5)$ & 17 & $=0.277$ \\
\hline ptc -1 & HA-in-1 & $f y-G F P-1$ & Cas-dsh-GFP & $2.01(1.5)$ & 20 & $=0.004$ \\
\hline ptc -1 & HA-in-1 & $f y-G F P-1$ & $\begin{array}{l}\text { Ubi-myc- } \\
\text { frtz-GFP }\end{array}$ & $5.30(5.3)$ & 10 & $=0.70$ \\
\hline
\end{tabular}

*number of copies

\& $=$ t-test comparison to ptc-GAL4 UAS-HA-in UAS-fy-GFP $/+$ 
Supplementary material: Figure S1. Accumulation of endogenous and overexpressed PPE proteins in pupal wing cells. All are confocal images that are single optical sections or maximal projections of a small number of optical sections from the sub-apical region. Panel A shows the asymmetric accumulation of Frtz-GFP when expressed from a ubiquitin promoter vector. Panel $\mathrm{B}$ shows the endogenous In protein detected by anti-In antibodies. $\mathrm{C}$ to $\mathrm{E}^{\prime}$ show the accumulation of Fy, In and Frtz when over expressed by ptc-Gal4. Note the wide spread accumulation of the over expressed proteins. 

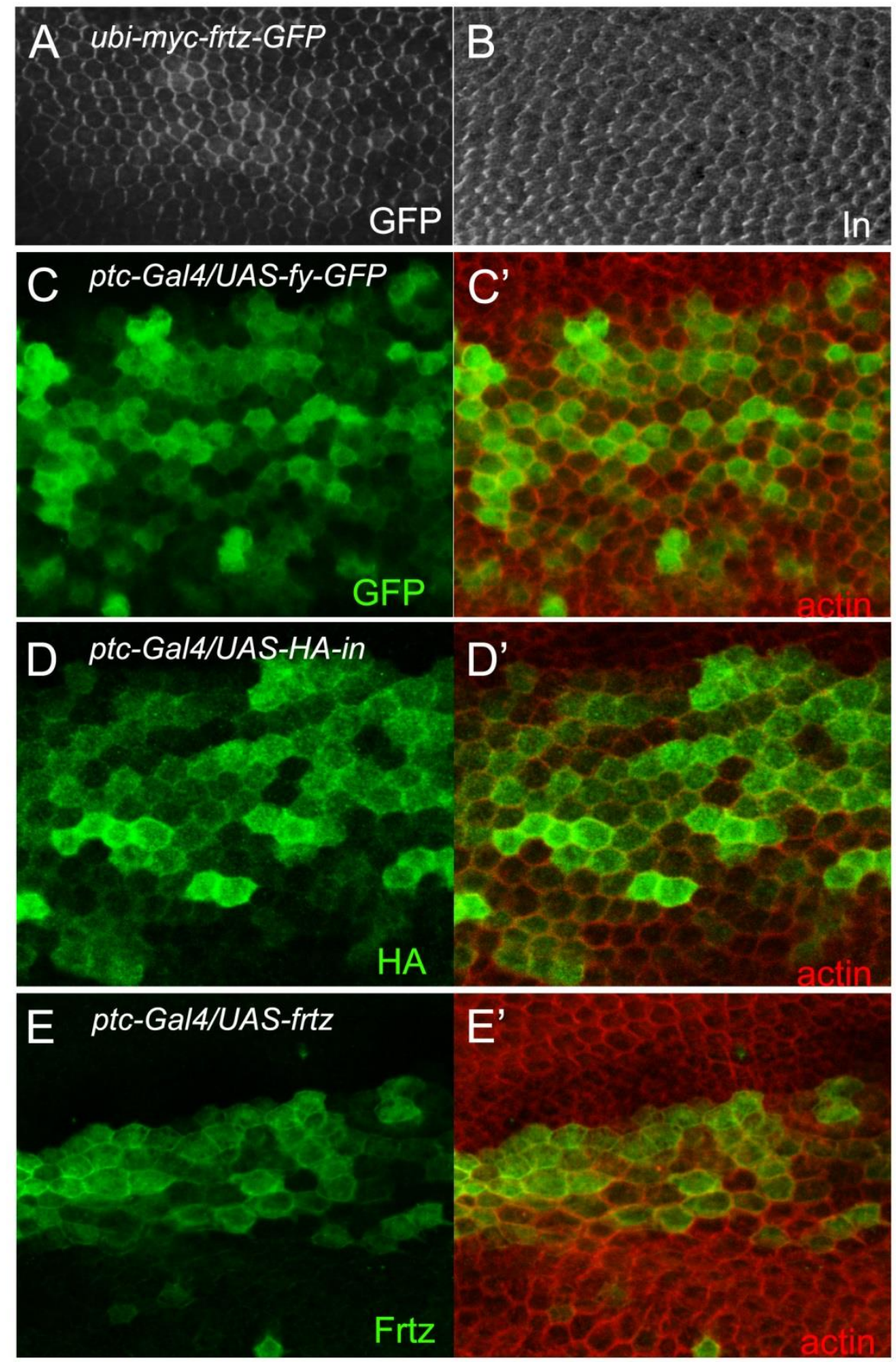
Supplementary material: Figure S2. The over expression of Frtz by ptc-Gal4 results in a fold in the wing. A ptc-Gal4/+; UAS-frtz wing is shown. The arrow points to the fold. 


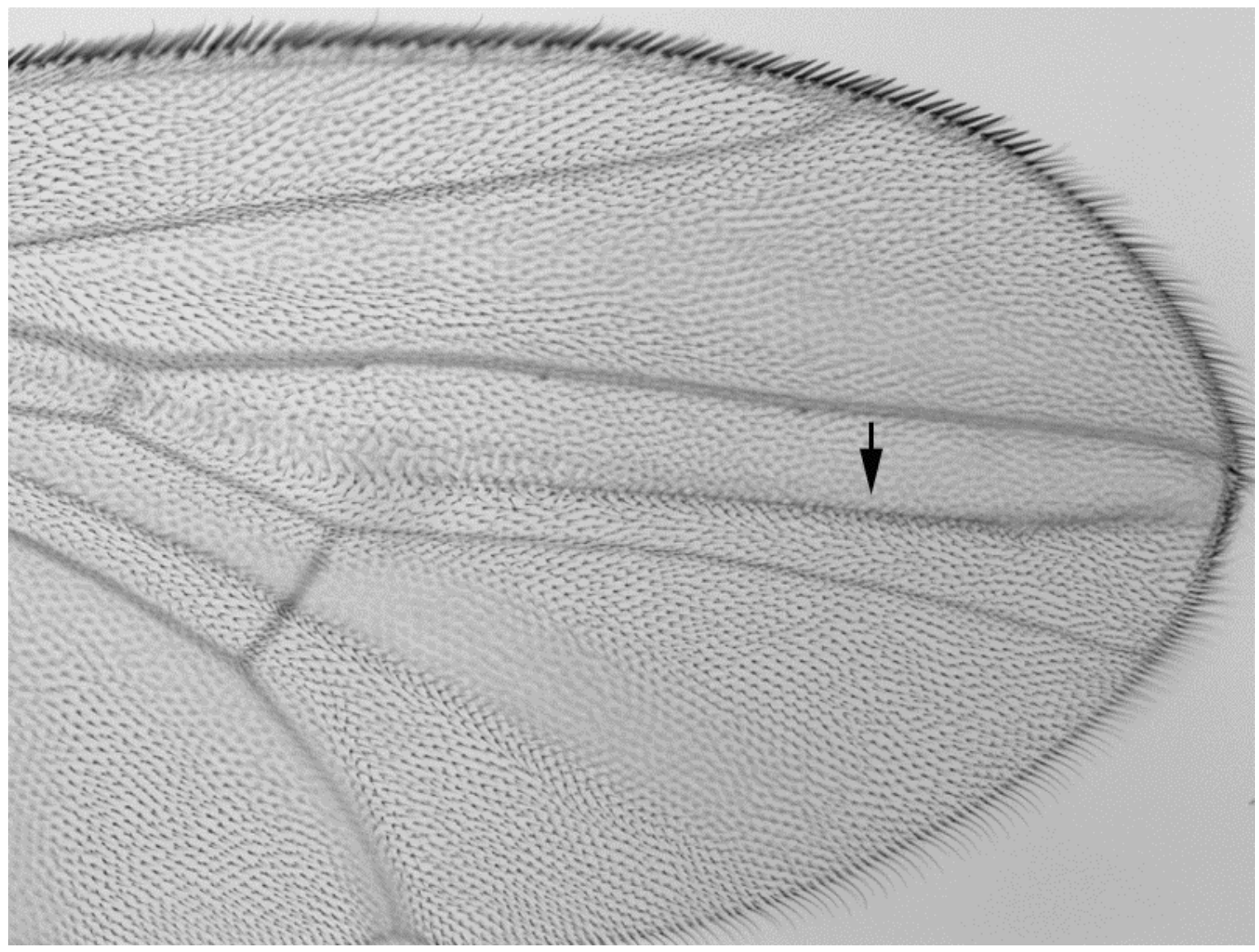


Supplementary material: Figure S3. Genetic interactions of $f z /$ stan pathway genes with frtz over expression. In the experiments described here the $U A S$-frtz transgene is not as highly expressed as the one used in Fig. 2. In A we scored each wing for the furthest region that showed a $45^{\circ}$ polarity disruption. In B we scored wings for the greatest extent of polarity disruption. $0-30^{\circ}=1$, $30^{\circ}-60^{\circ}=2$ and $>60^{\circ}=3$. The non-parametric Mann Whitney test was used to compare each condition to puc-Gal4 UAS-frtz/+ controls. 

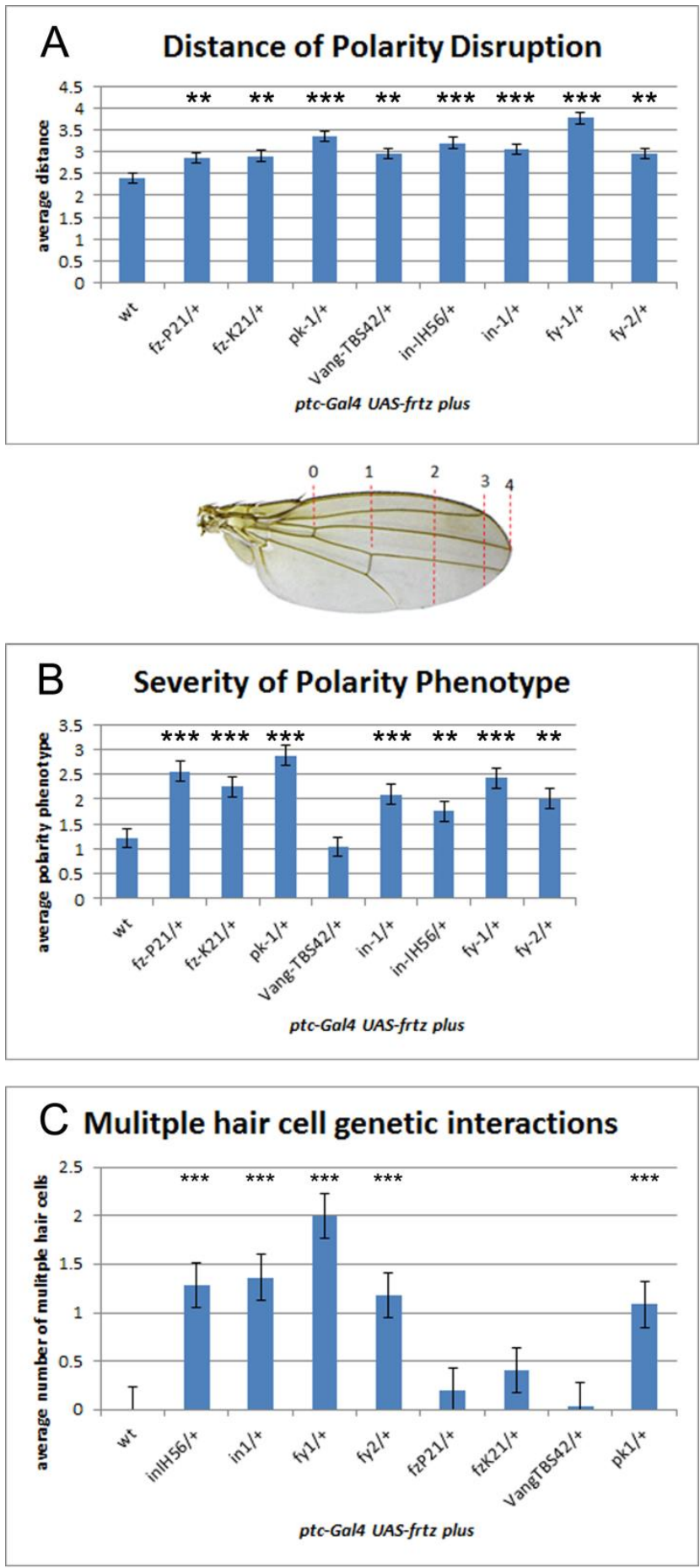

${ }^{*}=p<0.05,{ }^{* *}=p 0.05-.001,{ }^{* * *}=p<0.001$ 
Supplementary Material: Figure S4. The co-localization of In, Fy and Frtz. Shown is a ptcGal4/UAS-HA-in; UAS-myc-frtz/+ salivary gland (A-C) and pupal wing (D-F) immunostained for HA (red)(In) and myc (green) (Frtz). In the next row is a ptc-Gal4 UAS-HA-in UAS-fyGFP/+ salivary gland (GHI) and pupal wing (JKL) immunostained for GFP (green)(Fy) and HA (red)(In). In the third row is a ptc-Gal4/UAS-fy-GFP; UAS-myc-frtz/+ salivary gland (MNO) and pupal wing (PQR) immunostained for GFP (green) and Myc (red). The next row of images is a ptc-Gal4/UAS-HA-in UAS-fy-GFP; UAS-frtz-GFP pupal wing (STU) immunostained for HA (red) and GFP (green). Below (VWX) are higher magnification images of a small region of STU. Note the particulate staining in $(\mathrm{S}-\mathrm{X})$. The magnification in STU is the same as in DEF. 
ptc-Gal4/UAS-HA-in;UAS-(MYC) ${ }_{6}$-fitz

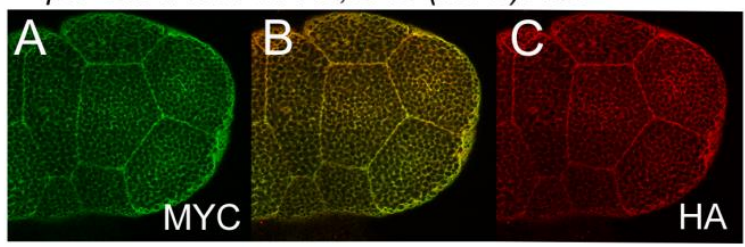

ptc-Gal4 UAS-HA-in UAS-fy-GFP

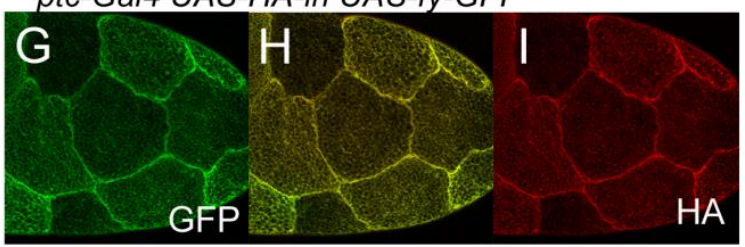

ptc-Gal4/UAS-fy-GFP; UAS-(MYC) $)_{6}$-fitz

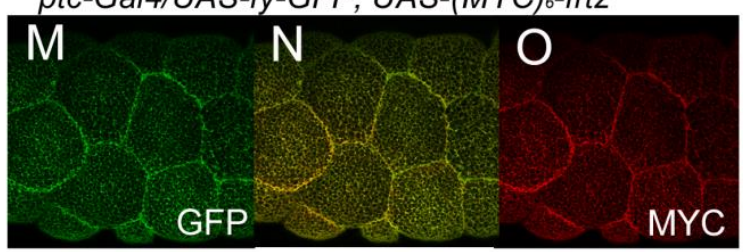

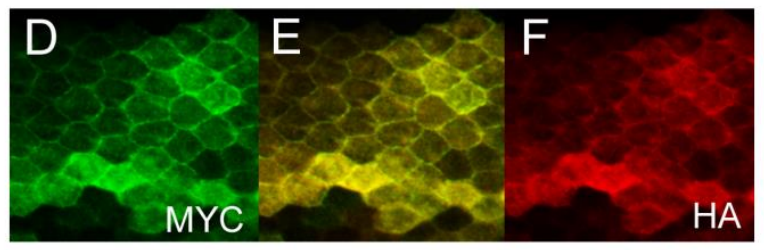
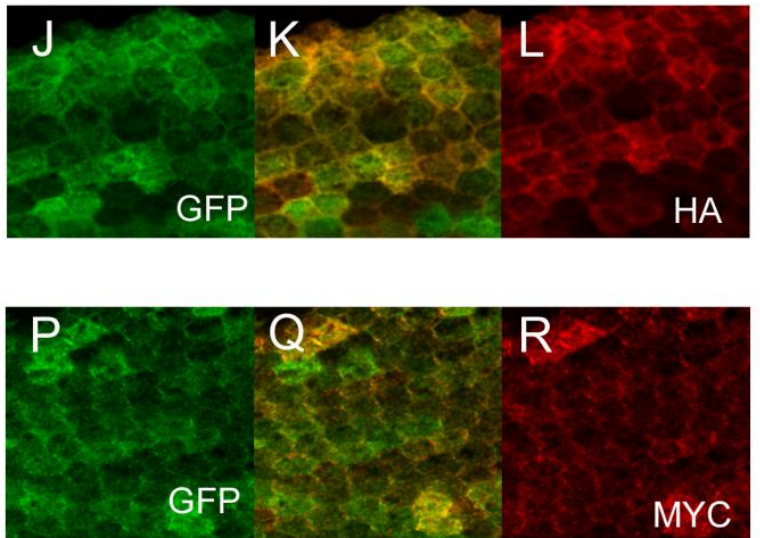

ptc-Gal4/UAS-in UAS-fy-GFP;UAS-frtz-GFP

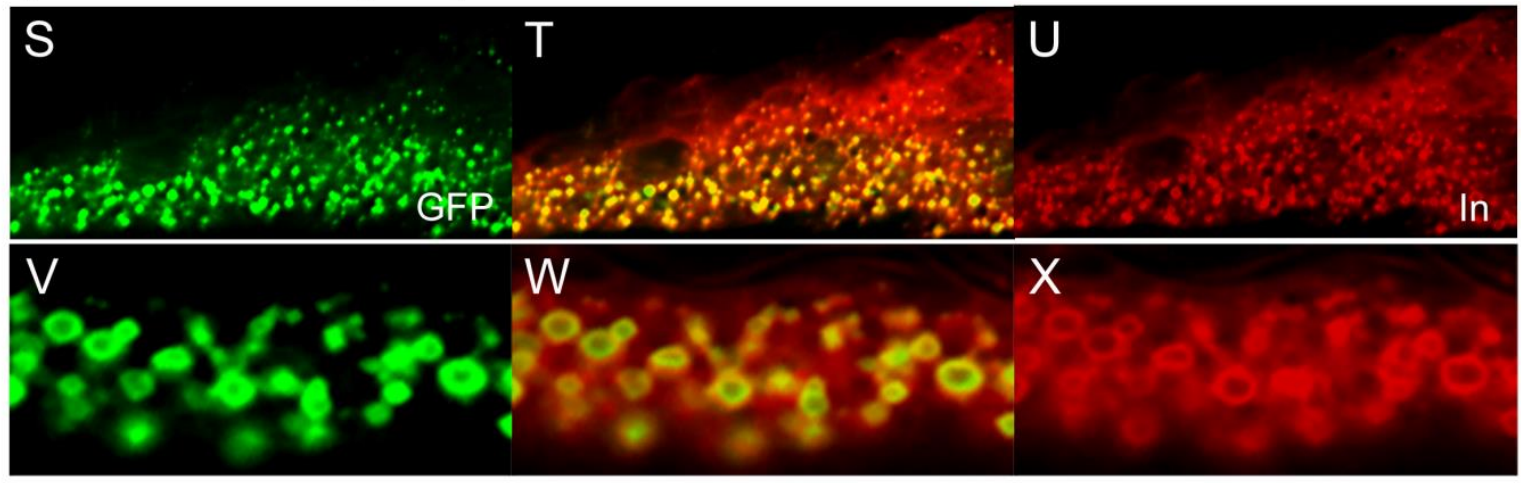


Supplementary material: Figure S5. Co-immunoprecipitation of PPE proteins. (A) S2 cell extracts where both HA-in and fy-Flag were expressed by the hsp70 promoter were tested by immunoprecipitation. The approximately $60 \mathrm{KD}$ band found in both the upper and lower western blots are presumably immunoglobin heavy chain contamination from the IP. Note that anti-In can pull down Fy and that anti-Flag can precipitate In. (B) Extracts of wing discs where ptcGal4 driven expression of HA-In and Fy-Flag-Ollas were tested for co-immunoprecipitation. Anti-HA was able to pull down Fy-Flag-Ollas and anti-Ollas was able to pull down In. (C) Wing disc extracts from ptc-Gal4 UAS-HA-in/+; UAS-frtz-GFP/+ animals were tested for coimmunoprecipitation. Anti-Frtz antibodies pulled down In. 

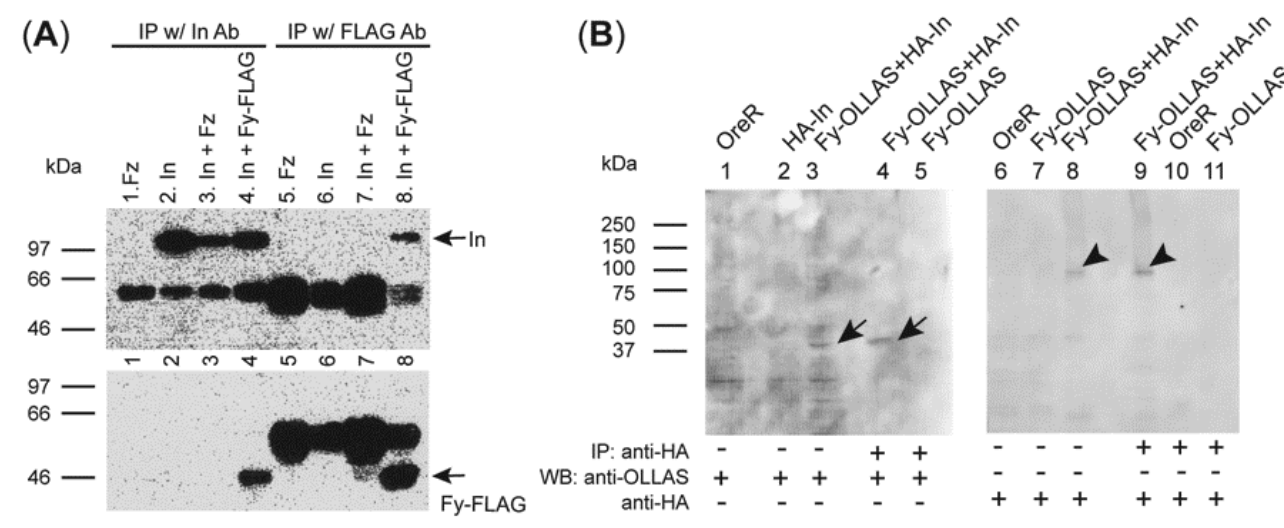

(C)

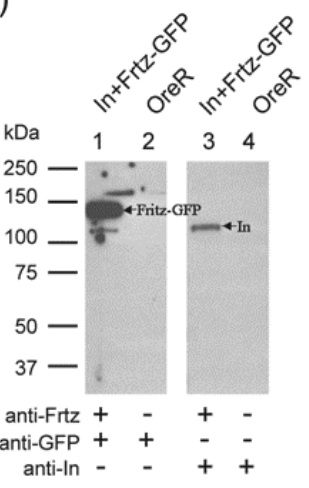


Supplementary material: Figure S6 Yeast two hybrid assays that provided evidence for In directly interacting with both Frtz and Fy. 


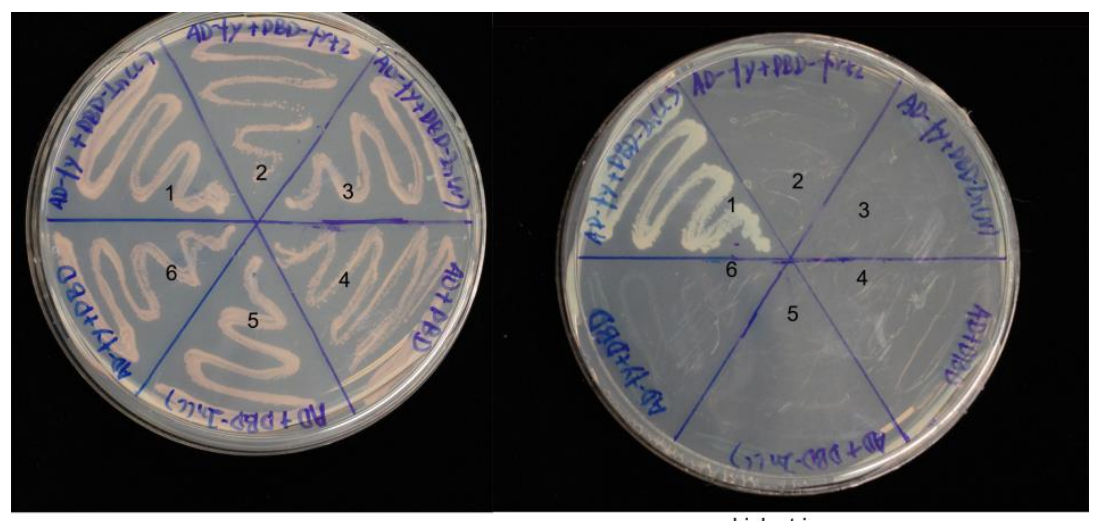

low stringency

2 aa dropout medium

high stringency

4 aa dropout medium

with $x$-alpha-gal

1: pGADT7- fy + pGBKT7- In (C teminus) 2: pGADT7-fy + pGBKT7- frtz

(colony will grow and turn blue if

3: pGADT7- fy + pGBKT7- In ( $\mathrm{N}$ terminus)

4: pGADT7 + pGBKT7

5: pGADT7 + pGBKT7 - In ( $C$ terminus)

6: pGADT7 - fy + pGBKT7

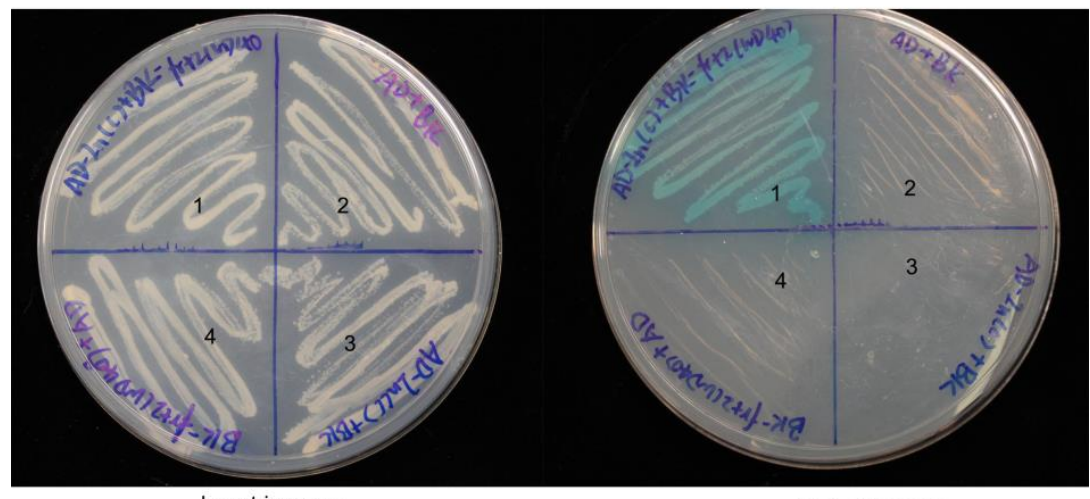

low stringency

(2 aa dropout medium)

high stringency

4 aa dropout medium

1: pGADT7 - In ( C terminus) + pGBKT7 - frtz (WD40)

2: pGADT7 + pGBKT7

3: pGADT7- In (C terminus) + pGBKT7

4: pGADT7 + pGBKT7- frtz (WD40)

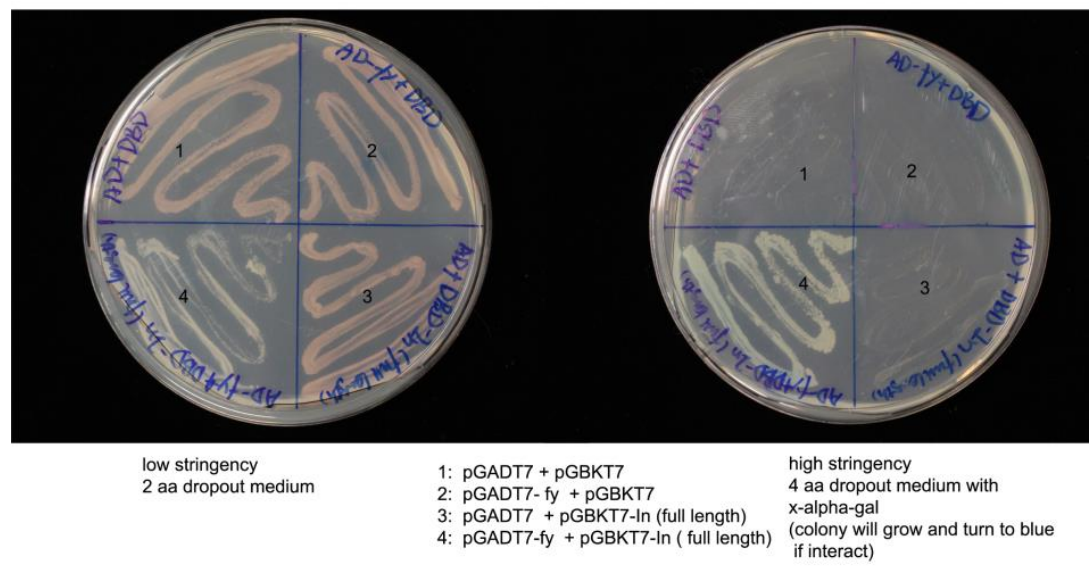

note: pGADT7 is $A D$ vector and pGBKT7 is DBD vector 
Supplementary material: Figure S7 The WD40 region of Frtz interacts with In. Shown is a summary of a set of yeast two hybrid experiments that indicate that the WD40 motif of Fritz interacts strongly with In. 
Dissecting the interaction between Frtz and In

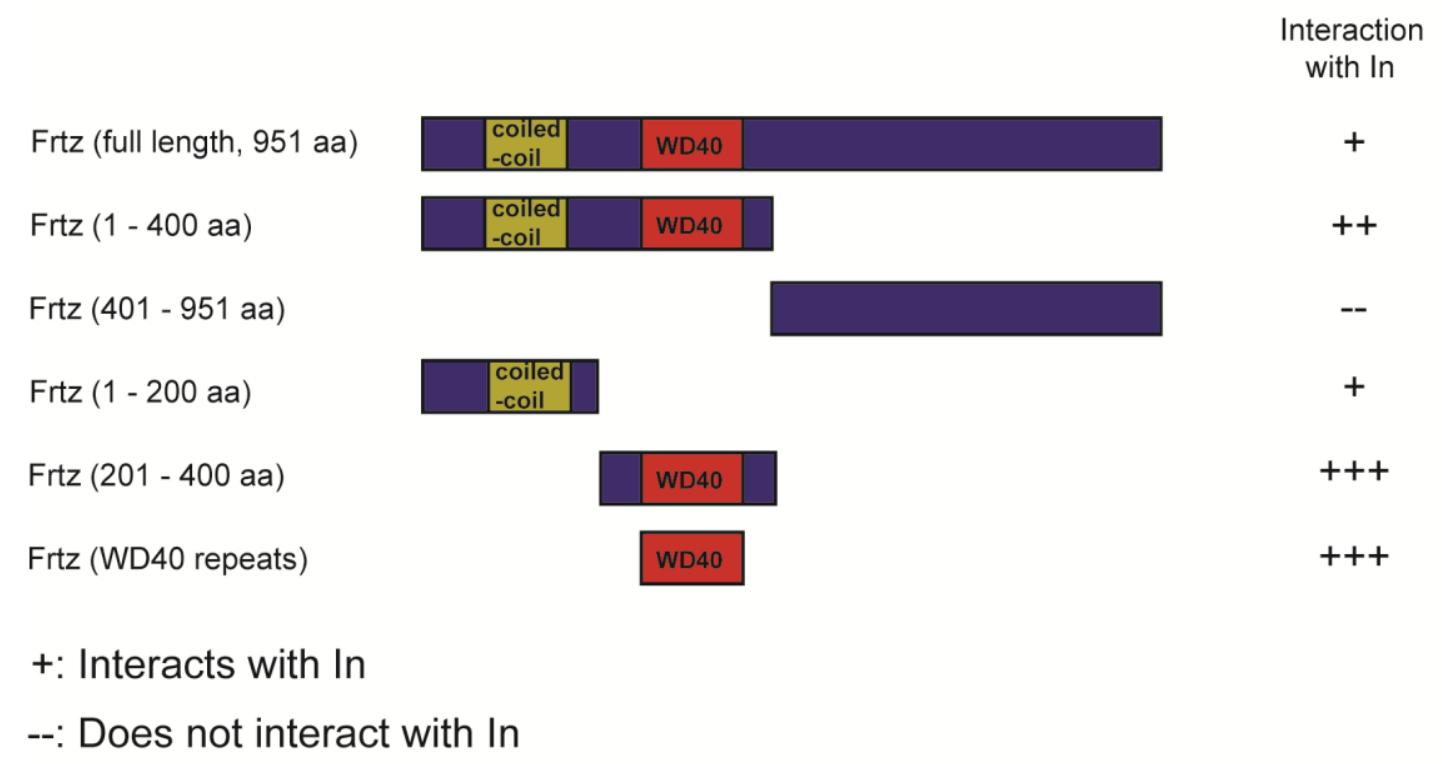




\section{Reference}

Adler, P.N. (1992). The genetic control of tissue polarity in Drosophila. Bioessays 14, 735-741.

Adler, P.N., Krasnow, R.E., and Liu, J. (1997). Tissue polarity points from cells that have higher Frizzled levels towards cells that have lower Frizzled levels. Curr Biol 7, 940-949.

Adler, P.N., Zhu, C., and Stone, D. (2004). Inturned localizes to the proximal side of wing cells under the instruction of upstream planar polarity proteins. Curr Biol 14, 2046-2051.

Amonlirdviman, K., Khare, N.A., Tree, D.R., Chen, W.S., Axelrod, J.D., and Tomlin, C.J. (2005). Mathematical modeling of planar cell polarity to understand domineering nonautonomy. Science 307, 423-426.

Axelrod, J.D. (2001). Unipolar membrane association of Dishevelled mediates Frizzled planar cell polarity signaling. Genes Dev 15, 1182-1187.

Axelrod, J.D., Miller, J.R., Shulman, J.M., Moon, R.T., and Perrimon, N. (1998). Differential recruitment of Dishevelled provides signaling specificity in the planar cell polarity and Wingless signaling pathways. Genes Dev 12, 2610-2622.

Babayeva, S., Rocque, B., Aoudjit, L., Zilber, Y., Li, J., Baldwin, C., Kawachi, H., Takano, T., and Torban, E. (2013). Planar cell polarity pathway regulates nephrin endocytosis in developing podocytes. J Biol Chem 288, 24035-24048. 
Bastock, R., Strutt, H., and Strutt, D. (2003). Strabismus is asymmetrically localised and binds to Prickle and Dishevelled during Drosophila planar polarity patterning. Development 130, 3007-3014.

Bischof, J., Maeda, R.K., Hediger, M., Karch, F., and Basler, K. (2007). An optimized transgenesis system for Drosophila using germ-line-specific phiC31 integrases. Proc Natl Acad Sci U S A 104, 3312-3317.

Boutros, M., Paricio, N., Strutt, D.I., and Mlodzik, M. (1998). Dishevelled activates JNK and discriminates between JNK pathways in planar polarity and wingless signaling. Cell 94, 109118.

Brand, A.H., and Perrimon, N. (1993). Targeted gene expression as a means of altering cell fates and generating dominant phenotypes. Development 118, 401-415.

Brooks, E.R., and Wallingford, J.B. (2012). Control of vertebrate intraflagellar transport by the planar cell polarity effector Fuz. J Cell Biol 198, 37-45.

Chae, J., Kim, M.J., Goo, J.H., Collier, S., Gubb, D., Charlton, J., Adler, P.N., and Park, W.J. (1999). The Drosophila tissue polarity gene starry night encodes a member of the protocadherin family. Development 126, 5421-5429. 
Chang, H., and Nathans, J. (2013). Responses of hair follicle-associated structures to loss of planar cell polarity signaling. Proc Natl Acad Sci U S A 110, E908-917.

Cho, K.O., Hunt, C.A., and Kennedy, M.B. (1992). The rat brain postsynaptic density fraction contains a homolog of the Drosophila discs-large tumor suppressor protein. Neuron 9, 929-942.

Collier, S., and Gubb, D. (1997). Drosophila tissue polarity requires the cell-autonomous activity of the fuzzy gene, which encodes a novel transmembrane protein. Development 124 , 4029-4037.

Collier, S., Lee, H., Burgess, R., and Adler, P. (2005). The WD40 repeat protein fritz links cytoskeletal planar polarity to frizzled subcellular localization in the Drosophila epidermis. Genetics 169, 2035-2045.

Cui, C., Chatterjee, B., Lozito, T.P., Zhang, Z., Francis, R.J., Yagi, H., Swanhart, L.M., Sanker, S., Francis, D., Yu, Q., et al. (2013). Wdpcp, a PCP protein required for ciliogenesis, regulates directional cell migration and cell polarity by direct modulation of the actin cytoskeleton. PLoS Biol 11, e1001720.

Das, G., Jenny, A., Klein, T.J., Eaton, S., and Mlodzik, M. (2004). Diego interacts with Prickle and Strabismus/Van Gogh to localize planar cell polarity complexes. Development 131, 44674476. 
Feiguin, F., Hannus, M., Mlodzik, M., and Eaton, S. (2001). The ankyrin repeat protein Diego mediates Frizzled-dependent planar polarization. Dev Cell 1, 93-101.

Goggolidou, P. (2014). Wnt and planar cell polarity signaling in cystic renal disease. Organogenesis 10, 86-95.

Goodrich, L.V., and Strutt, D. (2011). Principles of planar polarity in animal development. Development 138, 1877-1892.

Goto, T., and Keller, R. (2002). The planar cell polarity gene strabismus regulates convergence and extension and neural fold closure in Xenopus. Dev Biol 247, 165-181.

Gray, R.S., Abitua, P.B., Wlodarczyk, B.J., Szabo-Rogers, H.L., Blanchard, O., Lee, I., Weiss, G.S., Liu, K.J., Marcotte, E.M., Wallingford, J.B., et al. (2009). The planar cell polarity effector Fuz is essential for targeted membrane trafficking, ciliogenesis and mouse embryonic development. Nat Cell Biol 11, 1225-1232.

Groth, A.C., Fish, M., Nusse, R., and Calos, M.P. (2004). Construction of transgenic Drosophila by using the site-specific integrase from phage phiC31. Genetics $166,1775-1782$.

Gubb, D., and Garcia-Bellido, A. (1982). A genetic analysis of the determination of cuticular polarity during development in Drosophila melanogaster. J Embryol Exp Morphol 68, 37-57. 
Gubb, D., Green, C., Huen, D., Coulson, D., Johnson, G., Tree, D., Collier, S., and Roote, J. (1999). The balance between isoforms of the prickle LIM domain protein is critical for planar polarity in Drosophila imaginal discs. Genes Dev 13, 2315-2327.

Held, L.I., Jr., Duarte, C.M., and Derakhshanian, K. (1986). Extra joints and misoriented bristles on Drosophila legs. Prog Clin Biol Res 217A, 293-296.

Heydeck, W., and Liu, A. (2011). PCP effector proteins inturned and fuzzy play nonredundant roles in the patterning but not convergent extension of mammalian neural tube. Dev Dyn 240, 1938-1948.

Heydeck, W., Zeng, H., and Liu, A. (2009). Planar cell polarity effector gene Fuzzy regulates cilia formation and Hedgehog signal transduction in mouse. Dev Dyn 238, 3035-3042.

Jenny, A., Darken, R.S., Wilson, P.A., and Mlodzik, M. (2003). Prickle and Strabismus form a functional complex to generate a correct axis during planar cell polarity signaling. EMBO J 22, 4409-4420.

Jessen, J.R., Topczewski, J., Bingham, S., Sepich, D.S., Marlow, F., Chandrasekhar, A., and Solnica-Krezel, L. (2002). Zebrafish trilobite identifies new roles for Strabismus in gastrulation and neuronal movements. Nat Cell Biol 4, 610-615. 
Kim, S.K., Shindo, A., Park, T.J., Oh, E.C., Ghosh, S., Gray, R.S., Lewis, R.A., Johnson, C.A., Attie-Bittach, T., Katsanis, N., et al. (2010). Planar cell polarity acts through septins to control collective cell movement and ciliogenesis. Science 329, 1337-1340.

Klemenz, R., Weber, U., and Gehring, W.J. (1987). The white gene as a marker in a new Pelement vector for gene transfer in Drosophila. Nucleic Acids Res 15, 3947-3959.

Krasnow, R.E., and Adler, P.N. (1994). A single frizzled protein has a dual function in tissue polarity. Development 120, 1883-1893.

Lee, H., and Adler, P.N. (2002). The function of the frizzled pathway in the Drosophila wing is dependent on inturned and fuzzy. Genetics 160, 1535-1547.

Lu, Q., Yan, J., and Adler, P.N. (2010). The Drosophila planar polarity proteins inturned and multiple wing hairs interact physically and function together. Genetics $185,549-558$.

Montcouquiol, M., Rachel, R.A., Lanford, P.J., Copeland, N.G., Jenkins, N.A., and Kelley, M.W. (2003). Identification of Vangl2 and Scrb1 as planar polarity genes in mammals. Nature 423, 173-177.

Narimatsu, M., Bose, R., Pye, M., Zhang, L., Miller, B., Ching, P., Sakuma, R., Luga, V., Roncari, L., Attisano, L., et al. (2009). Regulation of planar cell polarity by Smurf ubiquitin ligases. Cell 137, 295-307. 
Park, T.J., Haigo, S.L., and Wallingford, J.B. (2006). Ciliogenesis defects in embryos lacking inturned or fuzzy function are associated with failure of planar cell polarity and Hedgehog signaling. Nat Genet 38, 303-311.

Park, W.J., Liu, J., Sharp, E.J., and Adler, P.N. (1996). The Drosophila tissue polarity gene inturned acts cell autonomously and encodes a novel protein. Development 122, 961-969. Pirrotta, V. (1988). Vectors for P-mediated transformation in Drosophila. Biotechnology 10, 437-456.

Sekelsky, J.J., Newfeld, S.J., Raftery, L.A., Chartoff, E.H., and Gelbart, W.M. (1995). Genetic characterization and cloning of mothers against dpp, a gene required for decapentaplegic function in Drosophila melanogaster. Genetics 139, 1347-1358.

Seo, J.H., Zilber, Y., Babayeva, S., Liu, J., Kyriakopoulos, P., De Marco, P., Merello, E., Capra, V., Gros, P., and Torban, E. (2011). Mutations in the planar cell polarity gene, Fuzzy, are associated with neural tube defects in humans. Hum Mol Genet 20, 4324-4333.

Strutt, D., and Warrington, S.J. (2008). Planar polarity genes in the Drosophila wing regulate the localisation of the FH3-domain protein Multiple Wing Hairs to control the site of hair production. Development 135, 3103-3111.

Strutt, D.I. (2001). Asymmetric localization of frizzled and the establishment of cell polarity in the Drosophila wing. Mol Cell 7, 367-375. 
Tabler, J.M., Barrell, W.B., Szabo-Rogers, H.L., Healy, C., Yeung, Y., Perdiguero, E.G., Schulz, C., Yannakoudakis, B.Z., Mesbahi, A., Wlodarczyk, B., et al. (2013). Fuz mutant mice reveal shared mechanisms between ciliopathies and FGF-related syndromes. Dev Cell 25, 623635.

Takeuchi, M., Nakabayashi, J., Sakaguchi, T., Yamamoto, T.S., Takahashi, H., Takeda, H., and Ueno, N. (2003). The prickle-related gene in vertebrates is essential for gastrulation cell movements. Curr Biol 13, 674-679.

Taylor, J., Abramova, N., Charlton, J., and Adler, P.N. (1998). Van Gogh: a new Drosophila tissue polarity gene. Genetics 150, 199-210.

Te Velthuis, A.J., Isogai, T., Gerrits, L., and Bagowski, C.P. (2007). Insights into the molecular evolution of the PDZ/LIM family and identification of a novel conserved protein motif. PLoS One 2, e189.

Tree, D.R., Shulman, J.M., Rousset, R., Scott, M.P., Gubb, D., and Axelrod, J.D. (2002). Prickle mediates feedback amplification to generate asymmetric planar cell polarity signaling. Cell 109, 371-381. 
Usui, T., Shima, Y., Shimada, Y., Hirano, S., Burgess, R.W., Schwarz, T.L., Takeichi, M., and Uemura, T. (1999). Flamingo, a seven-pass transmembrane cadherin, regulates planar cell polarity under the control of Frizzled. Cell 98, 585-595.

Wallingford, J.B., Vogeli, K.M., and Harland, R.M. (2001). Regulation of convergent extension in Xenopus by Wnt5a and Frizzled-8 is independent of the canonical Wnt pathway. Int J Dev Biol 45, 225-227.

Wang, J., Mark, S., Zhang, X., Qian, D., Yoo, S.J., Radde-Gallwitz, K., Zhang, Y., Lin, X., Collazo, A., Wynshaw-Boris, A., et al. (2005). Regulation of polarized extension and planar cell polarity in the cochlea by the vertebrate PCP pathway. Nat Genet 37, 980-985.

Wang, Y., Guo, N., and Nathans, J. (2006). The role of Frizzled3 and Frizzled6 in neural tube closure and in the planar polarity of inner-ear sensory hair cells. J Neurosci 26, 2147-2156.

Wang, Y., and Nathans, J. (2007). Tissue/planar cell polarity in vertebrates: new insights and new questions. Development 134, 647-658.

Wolff, T., and Rubin, G.M. (1998). Strabismus, a novel gene that regulates tissue polarity and cell fate decisions in Drosophila. Development 125, 1149-1159.

Wong, L.L., and Adler, P.N. (1993). Tissue polarity genes of Drosophila regulate the subcellular location for prehair initiation in pupal wing cells. J Cell Biol 123, 209-221. 
Yan, J., Huen, D., Morely, T., Johnson, G., Gubb, D., Roote, J., and Adler, P.N. (2008). The multiple-wing-hairs gene encodes a novel GBD-FH3 domain-containing protein that functions both prior to and after wing hair initiation. Genetics $180,219-228$.

Zhang, Z., Wlodarczyk, B.J., Niederreither, K., Venugopalan, S., Florez, S., Finnell, R.H., and Amendt, B.A. (2011). Fuz regulates craniofacial development through tissue specific responses to signaling factors. PLoS One 6, e24608.

Zilber, Y., Babayeva, S., Seo, J.H., Liu, J.J., Mootin, S., and Torban, E. (2013). The PCP effector Fuzzy controls cilial assembly and signaling by recruiting Rab8 and Dishevelled to the primary cilium. Mol Biol Cell 24, 555-565. 


\section{Chapter 3}

Planar cell polarity effector fritz has inturned independent function and interacts physically with the upstream core gene disheveled

Ying Wang 


\begin{abstract}
Planar cell polarity which is defined as the polarization of the cells within the plane of the epithelial sheet is an important feature of animal development. The epidermis of Drosophila melanogaster is decorated by a variety of polarized tissues and structures, such as wing hairs and sensory bristles. The genetic studies using Drosophila has uncovered a set of genes responsible for regulating planar cell polarity, and the genes comprise the frizzled/starry night signaling pathway. The genes were placed into three phenotypic and epistasis groups. inturned, fuzzy and fritz are planar cell polarity effectors, and they function downstream of the planar cell polarity core genes. Previous research showed proteins encoded by inturned, fuzzy and fritz accumulate asymmetrically on proximal side of the cell and form a protein complex through physical interaction with In. We also found a WD40 fragment in Frtz which is required for the interaction with In. In this chapter, I describe our attempts of mapping the amino acids which are important for this interaction in the Frtz WD40 fragment. However, 12 amino acids on the Frtz protein surface were mutated without blocking the interaction with In, and a frtz temperature sensitive hypomorphic allele, $f r t z^{3}$, didn't block the interaction with in as well. Also, we found frtz has both in dependent and independent functions. A mild overexpression of the Frtz protein partially rescued a newly generated in deletion stock $\left(i n^{p d}\right)$. We further found the distally accumulated planar cell polarity core gene $d s h$ physically interacts with both frtz and $f y$, and the antagonistic force between them results in the asymmetric accumulation of the protein complex containing planar cell polarity effectors on proximal edge of the wing cell. The interaction between Dsh and Frtz comes from multiple locations in Frtz protein, a 300 amino acids fragment on amino terminus and a 100 amino acids fragment toward carboxyl terminus.
\end{abstract}




\section{Introduction}

All epithelial cells display an apical-basal polarity. The basal surface is juxtaposed to an extracellular matrix that forms a basement membrane and the apical surface often forms a lumen or is exposed to the "outside". In addition, many epithelia are also polarized within the plane of the epithelium in which specialized structures are oriented within the plane of the epithelial sheet. Polarization of a field of cells within the plane of the cell sheet is defined as planar cell polarity (PCP). The epidermis of Drosophila melanogaster offers a variety of structures and tissues for studying PCP. The Drosophila wing is the best-characterized model system in part because of its simplicity. The adult wing is formed by the pupal wing that contains two layers of epithelial cells. Most wing cells are hexagonal in shape and each produces a single actin-rich hair or trichome at the distal vertex of the cell (Wong and Adler, 1993). The cuticular surface of the fly is decorated with many sensory bristles (Gho and Schweisguth, 1998; Lu et al., 1999). Adult sensory bristles are organized in a polarized fashion such that bristles on the thorax and abdomen point posteriorly, and on appendages they point distally. The polarized structures also include ommatidial clusters in the eye and epidermal hairs throughout the fly body (Singh et al., 2005; Tomlinson and Struhl, 1999; Turner and Adler, 1998; Wolff and Rubin, 1998; Wong and Adler, 1993; Zheng et al., 1995).

Much effort has been made to understand the mechanisms of PCP regulation during animal development. Genetic studies focused on the Drosophila wing led to the identification and characterization of a number of genes which are essential for PCP and comprise the frizzled $(f z) /$ starry night (stan) signal transduction pathway (Adler et al., 1998; Chae et al., 1999; Collier and Gubb, 1997; Collier et al., 2005; Feiguin et al., 2001; Gubb and Garcia-Bellido, 1982; 
Klingensmith et al., 1994; Strutt and Warrington, 2008; Taylor et al., 1998; Yang et al., 2002; Zeidler et al., 1999). Based on their functions in wing hair morphogenesis and epistasis experiments, these genes were placed into three phenotypic groups (Wong and Adler, 1993). The most upstream group is the PCP core group which functions universally for regulating PCP, including frizzled $(f z)$, disheveled (dsh), prickle/spiny leg ( $\mathrm{kk} / \mathrm{sple}$ ), van gogh (vang) (also known as strabismus), starry night (stan) (also known as flamingo) and diego (dgo) (Axelrod, 2001; Bastock et al., 2003; Das et al., 2004; Feiguin et al., 2001; Jenny et al., 2003; Strutt, 2001; Taylor et al., 1998; Tree et al., 2002; Usui et al., 1999). The PCP core genes loss-of-function (lof) mutations share a similar mutant phenotype

Most cells form a single hair with abnormal polarity and the site of hair initiation site is not restricted to the vicinity of the distal most vertex. Rather it tends to be located in a relatively central location on the apical surface. Downstream of the PCP core group are the PCP effector (PPE) genes whose lof mutations are epistatic to mutations in PCP core genes. The PPE group includes inturned (in), fuzzy (fy) and fritz (frtz) (Adler et al., 2004; Collier and Gubb, 1997; Collier et al., 2005; Wang et al., 2014; Wong and Adler, 1993). The PPE genes share very similar mutant phenotypes. Many wing cells produce multiples hairs (usually 2 to 3 hairs) and their polarity is disrupted. The multiple-wing-hair ( $m w h)$ gene is the only known member currently in the most downstream group (Lu et al., 2010; Wong and Adler, 1993; Yan et al., 2008b). mwh mutant cells generate more than 3 hairs per cell and their polarity is abnormal. However, this hierarchical structure may be context dependent, based on my recent study (Wang et al., 2014).We found that when over expressed the PPE proteins can alter both the subcellular location and level of accumulation of the upstream proteins. 
Proteins encoded by PCP genes accumulate asymmetrically on the proximal or distal edges of wing cells with the one exception that Stan accumulates on both (Usui et al., 1999). Vang, Pk, Frtz, In, Fy and Mwh accumulate on the proximal side (Adler et al., 2004; Bastock et al., 2003; Collier et al., 2005; Jenny et al., 2003; Strutt and Warrington, 2008; Tree et al., 2002; Wang et al., 2014), and Fz, Dsh and Dgo accumulate on the distal edge where hairs initiate (Axelrod, 2001; Das et al., 2004; Feiguin et al., 2001; Strutt, 2001). A variety of data show interactions between PCP proteins and PPE proteins are important for their proper functions (Adler et al., 2004; Axelrod, 2001; Das et al., 2004; Jenny et al., 2003; Tree et al., 2002; Usui et al., 1999; Wang et al., 2014; Zilber et al., 2013). There are at least two protein complexes formed within each cell, one on proximal side and another on distal side (Amonlirdviman et al., 2005; Tree et al., 2002). Asymmetric recruitment of PCP complexes relies on intercellular feedback at the interface of neighboring cells. That is, between the proximal edge of one cell and the distal edge of the cell it is juxtaposed to. Although the details remain to be established there is evidence that the two protein complexes bind each other by direct physical interaction between the transmembrane proteins Fz, Vang and Stan (Bastock et al., 2003; Chen et al., 2008; Das et al., 2002; Devenport and Fuchs, 2008; Lawrence et al., 2004; Shimada et al., 2001; Usui et al., 1999). Such interactions are thought to be able to propagate to neighboring cells ensuring a coherent pattern is established across the tissue. The transmembrane protein complexes found on the distal ( Fz and Stan) and proximal edges (Vang and Stan) then recruit the cytoplasmic core proteins, Dsh, Dgo and Pk to the proper side (Bastock et al., 2003; Das et al., 2004; Feiguin et al., 2001; Jenny et al., 2003; Jenny et al., 2005; Tree et al., 2002). Negative interactions between the cytoplasmic distal and proximal proteins reinforce the asymmetric pattern. For example, interaction between Pk and Dsh could prevent Dsh from accumulating on proximal 
side and Pk from accumulating on distal side (Bastock et al., 2003; Tree et al., 2002). The negative interaction may come from the proteasome-mediated degradation (Adler, 2012; Lecker et al., 2006; Narimatsu et al., 2009).

Various data show PPE proteins function in a protein complex mediated by direct physical interactions (Wang et al., 2014). In appears to be a key as it directly interacts with both Frtz and Fy. The interaction between In and Frtz comes from the WD40 repeats in Frtz protein and C-terminal half of In protein (Wang et al., 2014). In this chapter, I describe my attempts to map the amino acid residues in Frtz WD40 repeats responsible for interaction with In. Also, I describe a newly generated in precise deletion $\left(i n^{P D}\right)$ mutant that I generated using CRISPR/Cas9 genome editing technique. Using this in ${ }^{P D}$ stock, our genetic studies found that Frtz has In independent functions in Drosophila wing cells. The mild overexpression of Frtz partially rescues the ${ }^{P D}$ mutant, suggesting Frtz is partially able to bypass the need for In. I also describe the interaction between PCP core and PPE proteins. I found Dsh interacts with both Fy and Frtz proteins, and the interaction with Frtz comes from multiple locations in Frtz. 


\section{Methods and Materials}

\section{Fly genetics}

All flies were raised at $25^{\circ} \mathrm{C}$ unless otherwise stated. Mutant stocks were either obtained from the Bloomington Drosophila stock center at Indiana University, from the VDRC, generated in our lab or were generous gifts from J. Axelrod, D. Strutt, or T. Uemura. To direct transgene expression, we used the Gal4/UAS system (Brand and Perrimon, 1993). Ubi-myc-frtzGFP and UAS-myc-frtz were described in Chapter 2. We also generated transgenic lines that expressed myc-Frtz under the direction of $p W A L I U M$. For reasons that are unclear the myc-frtz transgene drove expression at a low level and did not result in a gain of function phenotype. Perhaps this is due to the amino terminal tag lowering protein accumulation. Dsh-myc stock was obtained from Bloomington Drosophila Stock Center at Indiana University (stock number 25385).

\section{Antibodies}

Anti-Myc antibody was obtained from Cell Signaling Technology. Anti-GFP antibody was obtained from Molecular Probes. Alexa 488- and Alexa 568-conjugated secondary antibodies were purchased from Molecular Probes. Anti-Frtz antibody was generated in rats using a Frtz protein made in E. coli and his tagged to facilitate purification. A similar strategy was used to obtain an anti-Fy antibody. In both cases the expressed proteins were found in inclusion bodies and solubilized and we do not know if they were in a native conformation. Neither antibody was able to detect the endogenous protein.

\section{Immunostaining}


A standard paraformaldehyde fixation and staining procedure was used (Adler et al., 2004). Briefly, fly pupae were fixed in $4 \%$ paraformaldehyde, PBS for $2-3 \mathrm{hr}$ at $4^{\circ} \mathrm{C}$. After fixation, wings were dissected, rinsed, blocked and then stained with primary antibodies in PBS, $0.3 \%$ Triton X-100 and 10\% goat-serum overnight at $4{ }^{\circ} \mathrm{C}$. Secondary antibodies were applied for 2-3 hr at room temperature.

\section{Plasmid constructs}

in $^{P D}$ CRISPR/Cas9 gRNA plasmid construction

5 gRNA primer:

5 gRNA 5': CTTCGGGAAACTCAAAACCCACCG

5 gRNA 3': AAACCGGTGGGTTTTGAGTTTCCC

3 gRNA primer:

3 gRNA 5' : CTTCGTCTTTTGACTTCTGGTGCG

3 gRNA 3' : AAACCGCACCAGAAGTCAAAAGAC

Oligos were phosphorylated using T4 PNK. Then complementary primers were selfannealed by running the following thermocycler program: $95^{\circ} \mathrm{C}$ for $5 \mathrm{~min}$, then ramp to $25^{\circ} \mathrm{C}$ at a rate of $-5^{\circ} \mathrm{C} / \mathrm{min}$. The $p U 6-\mathrm{BbsI}$-chiRNA (Addgene) plasmid was digested with BbsI enzyme and de-phosphorylated. The oliogs were mixed with cut $p U 6-B b s I-c h i R N A$ plasmid, the mix ligated and transformed into E. coli competent cell.

in $^{P D}$ CRISPR/Cas9 HDR template 
Primers for 5' and 3' inturned flanking region

In5 flank 5 - CCGGAATTCCAGCAGACAACTGTTCCCAT (EcoRI)

In5 flank 3 - CTAGCTAGCAAGCCGAGATCCGATCTCAG (NheI)

In3 flank 5 - AACTGCAGAACCAATGCATTGGAAACATCGTGCGAATGTGTT (PstI)

In3 flank 3 - CCGCTCGAGCGGAGCACATGGTTTTGGTCTCT (XhoI)

Both 5' and 3' flanking regions (about $1.5 \mathrm{~kb}$ each) were cloned by PCR using genomic DNA and digested with corresponding restriction enzyme. pHD-DsRed (Addgene) vector was digested with the same restriction enzymes and ligated with both flanking regions.

Transgenic DNA injection was performed at Rainbow Transgenic Flies, Inc. Both gRNA plasmids and HDR repair template were injected into Nos-cas9 attp40 (18055). The Go progeny were crossed with $i n^{I H 56}$ and screened for offspring with an in phenotype. Stocks were established of the new mutant allele and that was confirmed by genomic DNA PCR and a DsRed fluorescent signal.

\section{Construct subcloning for two-hybrid assays}

Full-length in cDNA was subcloned into $p G A D T 7$ vector from NdeI-BamHI. The following primers were used:

Inturn-th5: TCTAGGGAATTTCCATATGCGCAAATCGCCGGCCAG;

Inturn-th3: GATCGCGGATCCATGTCATCCCATTGAGAAGAAGGA. 
Full-length $f y$ cDNA was subcloned into $p G A D T 7$ vector from NcoI-BamHI. The following primers were used:

pGBKT7-5': CATGCCATGGAGATGTCCATCTATTTGTTATG;

pGBKT7-3': CGCGGATCCTTATCACCAACATACTGACTTC;

Full-length of fritz cDNA was subcloned into $p G B K T 7$ vector from NdeI-EcoRI . The following primers were used:

5' frtz-hybrid: GGGAATTCCATATGCTGCTCAGCGAGACC;

3'fritz-hybrid: CCGGAATTCTTATTAGACCACGCCGAAGTGGA.

Full-length $d s h$ cDNA was subcloned into both $p G B K T 7$ and $p G A D T 7$ vectors from NdeI-BamHI. The following primers were used:

AD/Bk-Dsh5':CATATGGACGCGGACAGGGGCGG

AD/Bk-Dsh 3': GGATCCCTACAATACGTAATTAAATACGG

To get truncated forms of fritz cDNA, we used the following primers and the PCR products were subcloned into $p G B K T 7$ vector using NdeI and EcoRI restriction sites.

1) Primers for $p G B K T 7-f r t z(1-400 \mathrm{aa})$

5' frtz-hybrid: GGGAATTCCATATGCTGCTCAGCGAGACC;

DBD-frtzN-3’: CCGGAATTCTTAGTGCGACAGATCCAGCAAG 
2) Primers for $p G B K T 7-f r t z$ (401 aa-end)

DBD-frtzC-5': GGGAATTCCATATGTACTTCGTGGCCCAGCCA

3'fritz-hybrid: CCGGAATTCTTATTAGACCACGCCGAAGTGGA.

3) Primers for $p G B K T 7-f r t z$ (201-400aa)

Dfrtz400-5’: GGGAATTCCATATGAACGCCAGCTTCGATC

DBD-frtzN-3': CCGGAATTCTTAGTGCGACAGATCCAGCAAG

4) Primers for $p G B K T 7-f r t z$ (WD40:308aa-385aa)

DBD-Fritz-WD5: GGAATTCCATATGCAGATCTGCTCCTTTGCCTTC

DBD-Fritz-WD3: CGGAATTCTTACAAGCTGATGGCCAATGGTG

5) Primers for $p G B K T 7-f r t z$ (100-700aa)

300 primer 5 - GGAATTCCATATGCTCTTCTCACACGGCCTGAT

2100 primer 3 - CCGGAATTCCTCGCCAGCGAAGATGTTGG

6) Primers for $p G B K T 7-f r t z$ (200-600aa)

600 primer 5 - GGAATTCCATATGAACGCCAGCTTCGATCTGTT

1800 primer 3 - CCGGAATTCTCGTGGATTATGGCAGCTGC

7) Primers for $p G B K T 7-f r t z$ (300-500aa) 
900 primer 5 - GGAATTCCATATGACCTCCATTCCAATGGGCGC

1500 primer 3 - CCGGAATTCACATAGTTGGCTATCTTGTG

\section{Frtz fragment deletion assay constructs}

Each fragment deletion construct carried a 100 aa fragment deletion (except the last one which has 151aa deletion) of the full length frtz cDNA. In addition, there is a 26 aa spacer between the two frtz fragments, including a myc tag and several random aa.

1) $p G B K T 7-f r t z$ (100-951aa). Cut $p G B K T 7$ vector with NdeI and Xmal, and perform the Gibson assembly. The following primers were used to amplify the fragments by PCR.

frtz 100-951 5' ATCTCAGAGGAGGACCTGCATATGTTCTCACACGGCCTGATC

frtz 100-951 3' GGTCGACGGATCCCCGGGTTATTAGACCACGCCGAAGTGGAC

2) $p G B K T 7-f r t z$ (1-100aa \& 201-951aa). Cut $p G B K T 7$ vector with XhoI and Xmal, and perform the Gibson assembly. The following primers were used to amplify the fragments by PCR.

Primers for amplifying 1-100aa fragment:

frtz 1-100 200-951 1st 5' CAGCTATTTCTACTGATTTTTCCTCGAGAAGACCTTGACATG

frtz 1-100 200-951 1st 3' CAGGTCTTCTTCTGAGATTAGCTTTTGTTCTTCGCCGTGTGA GAAGAGGAGCAG

Primers for amplifying 201-951aa fragment 
frtz 1-100 200-951 2nd 5' GAAGAACAAAAGCTAATCTCAGAAGAAGACCTGCGCCACC TCACAGTTAACGCC

frtz 1-100 200-951 2nd 3' GCCGCTGCAGGTCGACGGATCCCCGGGTTATTAGACCACGC CGAAGTGGAC

3) $p G B K T 7-f r t z$ (1-200aa \& 301-951aa). Cut $p G B K T 7$ vector with XhoI and Xmal, and perform the Gibson assembly. The following primers were used to amplify the fragments by PCR

Primers for amplifying 1-200aa fragment

frtz 1-200 300-951 1st 5' (same with frtz 1-100 200-951 1st 5' ) CAGCTATTTCTACTGATTT TTCCTCGAGAAGACCTTGACATG

frtz 1-200 300-951 1st (same with frtz 1-100 200-951 2nd 3') 3'CAGGTCTTCTTCTGAGATT AGCTTTTGTTCTTCATCGAAGCTGGCGTTAACTGT

Primers for amplifying 301-951 fragment

frtz 1-200 300-951 2nd 5'GAAGAACAAAAGCTAATCTCAGAAGAAGACCTGCAGCGCA CCGCCATTACCTCC

frtz 1-200 300-951 2nd (same with frtz 1-100 200-951 2nd 3') GCCGCTGCAGGTCGACGG ATCCCCGGGTTATTAGACCACGCCGAAGTGGAC 
4) $p G B K T 7-f r t z$ (1-300aa \& 401-951aa). Cut $p G B K T 7$ vector with XhoI and Xmal, and perform the Gibson assembly. The following primers were used to amplify the fragments by PCR

Primers for amplifying 1-300aa fragment

frtz 1-300 400-951 1st 5': (same with frtz 1-100 200-951 1st 5' ) CAGCTATTTCTACTGATTT TTCCTCGAGAAGACCTTGACATG

frtz 1-300 400-951 1st 3': CAGGTCTTCTTCTGAGATTAGCTTTTGTTCTTCCATTGGAAT GGAGGTAATGGC

Primers for amplifying 401-951 fragment

frtz 1-300 400-951 2nd 5': GAAGAACAAAAGCTAATCTCAGAAGAAGACCTGCTGGATC TGTCGCACTACTTC

frtz 1-300 400-951 2nd 3': (same with frtz 1-100 200-951 2nd 3') GCCGCTGCAGGTCGACG GATCCCCGGGTTATTAGACCACGCCGAAGTGGAC

5) $p$ GBKT7-frtz (1-400aa \& 501-951aa). Cut $p G B K T 7$ vector with XhoI and Xmal, and perform the Gibson assembly. The following primers were used to amplify the fragments by PCR

Primers for amplifying 1-400aa fragment

frtz 1-400 500-951 1st 5' : (same with frtz 1-100 200-951 1st 5' ) CAGCTATTTCTACTGATTT TTCCTCGAGAAGACCTTGACATG 
frtz 1-400 500-951 1st 3' : CAGGTCTTCTTCTGAGATTAGCTTTTGTTCTTCCTGGGCCA CGAAGTAGTGCGA

Primers for amplifying 501-951 fragment

frtz 1-400 500-951 2nd 5': GAAGAACAAAAGCTAATCTCAGAAGAAGACCTGTGCCTG ATTACGCTGCACAAG

frtz 1-400 500-951 2nd 3': (same with frtz 1-100 200-951 2nd 3') GCCGCTGCAGGTCGACG GATCCCCGGGTTATTAGACCACGCCGAAGTGGAC

6) $p$ GBKT7-frtz (1-500aa \& 601-951aa). Cut $p G B K T 7$ vector with XhoI and Xmal, and perform the Gibson assembly. The following primers were used to amplify the fragments by PCR

Primers for amplifying 1-500aa fragment

frtz 1-500 600-951 1st 5': (same with frtz 1-100 200-951 1st 5' ) CAGCTATTTCTACTGATTT TTCCTCGAGAAGACCTTGACATG

frtz 1-500 600-951 1st 3': CAGGTCTTCTTCTGAGATTAGCTTTTGTTCTTCGTTGGCTAT CTTGTGCAGCGT

Primers for amplifying 601-951aa fragment

frtz 1-500 600-951 2nd 5': GAAGAACAAAAGCTAATCTCAGAAGAAGACCTGGTGGCCT TCAGTCAGGCAGCT 
frtz 1-500 600-951 2nd 3': (same with frtz 1-100 200-951 2nd 3') GCCGCTGCAGGTCGACG G ATCCCCGGGTTATTAGACCACGCCGAAGTGGAC

7) $p G B K T 7-f r t z$ (1-600aa \&701-951aa). Cut $p G B K T 7$ vector with XhoI and Xmal, and perform the Gibson assembly. The following primers were used to amplify the fragments by PCR

Primers for amplifying 1-600aa fragment

frtz 1-600 700-951 1st 5': (same with frtz 1-100 200-951 1st 5' ) CAGCTATTTCTACTGATTT TTCCTCGAGAAGACCTTGACATG

frtz 1-600 700-951 1st 3': CAGGTCTTCTTCTGAGATTAGCTTTTGTTCTTCGATTATGGC AGCTGCCTGACT

Primers for amplifying 700-951aa fragment

frtz 1-600 700-951 2nd 5': GAAGAACAAAAGCTAATCTCAGAA GAAGACCTGGTTTCA ATAGCGCCGCCAACA

frtz 1-600 700-951 2nd 3': (same with frtz 1-100 200-951 2nd 3') GCCGCTGCAGGTCGACG GATCCCCGGGTTATTAGACCACGCCGAAGTGGAC

8) $p G B K T 7-f r t z$ (1-700aa \&801-951aa). Cut $p G B K T 7$ vector with XhoI and Xmal, and perform the Gibson assembly. The following primers were used to amplify the fragments by PCR

Primers for amplifying 1-700aa fragment 
frtz 1-700 800-951 1st 5': (same with frtz 1-100 200-951 1st 5' ) CAGCTATTTCTACTGATTT TTCCTCGAGAAGACCTTGACATG

frtz 1-700 800-951 1st 3': CAGGTCTTCTTCTGAGATTAGCTTTTGTTCTTCCAGCGAAGA TGTTGGCGGCGC

Primers for amplifying 801-951aa fragment

frtz 1-700 800-951 2nd 5': GAAGAACAAAAGCTAATCTCAGAAGAAGACCTGGCCAT GCTGCCATCGCTCACC

frtz 1-700 800-951 2nd 3': (same with frtz 1-100 200-951 2nd 3') GCCGCTGCAGGTCG ACGGATCCCCGGGTTATTAGACCACGCCGAAGTGGAC

9) $p G B K T 7-f r t z$ (1-800aa). Cut $p G B K T 7$ vector with XhoI and Xmal, and perform the Gibson assembly. The following primers were used to amplify the fragments by PCR.

frtz 1-800 5': (same with frtz 1-100 200-951 1st 5' )

CAGCTATTTCTACTGATTTTTCCTCGAGAAGACCTTGACATG

frtz 1-800 3': GCAGGTCGACGGATCCCCGGGTTATTACGATGGCAGCATGGCGGG

\section{Human PCP homologs yeast-two-hybrid constructs}

Full-length of intu cDNA was subcloned into both $p G B K T 7$ and $p G A D T 7$ vectors from EcoRI-BamHI. The following primers were used:

Intu 5': CCGGAATTCATGGCCTCTGTGGCTTCGTGC (EcoRI) 
Intu 3': CGCGGATCCCTACAAGGTTAACCCAAAGAA (BamHI)

Full-length of $w d p c p$ cDNA was subcloned into both $p G B K T 7$ and $p G A D T 7$ from NdeIEcoRI. The following primers were used:

WDPCP 5' GGAATTCCATATGATGTTTTCGTCTCTACATTCAGC (NdeI)

WDPCP 3' CCGGAATTCTTACACCAGACCAAAGTGAATCAT (EcoRI)

Full-length of $d v l 2$ cDNA was subcloned into both $p G B K T 7$ and $p G A D T 7$ vectors from NdeI-XhoI. The following primers were used:

dvl2 5' GGAATTCCATATGATGGCGGGTAGCAGCACTGG (ndeI)

dvl2 3' CCGCTCGAGCTACATAACATCCACAAAGAACTC (XhoI)

For the yeast two hybrid assays, we used yeast strain AH109 and Matchmaker TwoHybrid System 3 from Clontech. Briefly, full length or truncated cDNA was subcloned into $p G A D T 7$ or $p G B K T 7$ using specific restriction enzyme sites or Gibson assembly. To test the interaction of defined protein partners, $p G A D T 7$ and $p G B K T 7$ plasmids containing one of the two protein partners were co-transformed into yeasts. Transformed yeasts were selected by growing on two-marker dropout medium (SD/-Leu/-Trp). These two markers select for the presence of both plasmids. Colonies from the previous dropout medium were transferred to a four-marker dropout medium (SD/-Ade/-His/-Leu/-Trp) with X-alpha-Gal. Only colonies growing on four-marker plates were considered as evidence for a positive interaction.

\section{Quick Preparation of Genomic DNA from Drosophila}


Buffer A (store at room temperature)

$100 \mathrm{mM}$ Tris- $\mathrm{Cl}(\mathrm{pH} 7.5)$

100 mM EDTA

$100 \mathrm{mM} \mathrm{NaCl}$

$0.5 \%$ SDS

Buffer B (store at $4^{\circ} \mathrm{C}$ )

$200 \mathrm{~mL}$ potassium acetate $(5 \mathrm{M})$

$500 \mathrm{~mL}$ lithium chloride $(6 \mathrm{M})$

Mix together and store at $4^{\circ} \mathrm{C}$.

1. Collect 30 anesthetized flies in a $1.5-\mathrm{mL}$ microcentrifuge tube placed on ice. Flies can be stored at $-80^{\circ} \mathrm{C}$ indefinitely or DNA can be prepared immediately without freezing the flies.

2. Grind flies in $200 \mu \mathrm{L}$ of Buffer A with a disposable tissue grinder. Add an additional $200 \mu \mathrm{L}$ of Buffer A (to a total volume of $400 \mu \mathrm{L}$ ) and continue grinding until only cuticles remain ( 1-2 min, grinding by hand).

3. Incubate samples at $65^{\circ} \mathrm{C}$ for $30 \mathrm{~min}$.

4. Add $800 \mu \mathrm{L}$ of Buffer B to each sample, mix well by inverting the tube multiple times, and incubate on ice for at least $10 \mathrm{~min}$ and up to a few hours. 
5. Centrifuge in a microcentrifuge at $12,000 \mathrm{rpm}$ for $15 \mathrm{~min}$ at room temperature.

6. Transfer $1 \mathrm{~mL}$ of the supernatant into a new microcentrifuge tube (some of the precipitate will not pellet, but instead float on top of the supernatant; be extremely careful to avoid transferring any floating precipitate). Discard the pellet. If necessary, repeat the centrifugation step to remove any contaminating precipitate.

7. Add $600 \mu \mathrm{L}$ of isopropanol to each sample, and mix well by inverting the tube several times.

8. Centrifuge at $12,000 \mathrm{rpm}$ for $15 \mathrm{~min}$ at room temperature.

9. Discard the supernatant. Wash the pellet with $70 \%$ ethanol, air-dry, and re-suspend in $150 \mu \mathrm{L}$ of TE buffer.

10. Store the DNA at $-20^{\circ} \mathrm{C}$.

\section{Coimmunoprecipitation and western blotting}

The following genotypes were used in these experiments:

w; ptc-Gal4 dsh-myc; UAS-fy-GFP

w; ptc-Gal4 dsh-myc;UAS-(myc) $)_{6}-f r t z$

Co-immunoprecipitation experiments were done in a variety of different ways. The most common procedure was as follows. 150-200 wing discs of transgenic flies were dissected from third instar larvae and homogenized in pre-chilled lysis buffer containing protease inhibitors. The extract was then spun to remove cell debris. Protein A agarose beads (Roche) were added to 
the extract and incubated for at least $3 \mathrm{hr}$ to reduce the background of non-specific binding. The extract was spun again and $7-10 \mu l$ desired antibody was added to the supernatant and incubated for 3-4 hr. New protein A agarose beads were then added and the sample was incubated overnight. The beads were pelleted and washed. Protein was released from the beads and analyzed by standard western blot procedures.

\section{Imaging}

Adult wings were mounted in Euparal and bright field images obtained using a Spot RT camera and a Zeiss Axiskop II microscope. In most cases a 40X Apochromatic objective was used (oil, 1.4 NA). Live imaging of all Frtz protein was obtained using a Zeiss 780 confocal microscope, $63 \mathrm{x}$ or $40 \mathrm{x}$ oil immersion lens. Final panels were assembled using Adobe Illustrator or Microsoft PowerPoint. 


\section{Results}

\section{Mapping the residues responsible for interaction between In \& Frtz}

Our yeast-two-hybrid and co-immunoprecipitation assays show In physically interacts with Frtz (Chapter 2). Various Frtz fragments were tested to study the interaction with In protein. One fragment from a.a.304 to a.a.384 of Frtz protein showed the strongest interaction with In and the interaction was stronger than full length Frtz. There are two tandem WD40 repeats found in this fragment (Collier S et al., 2005), so it was named as Frtz WD40 fragment. In order to know which amino acid residues are responsible for this interaction, Frtz WD40 fragment mapping assay was done using site directed mutagenesis and yeast-two-hybrid system.

The WD40 repeat is a short structural motif of approximately 40 amino acids, often terminating in a tryptophan-aspartic acid (W-D) dipeptide (Neer et al., 1994). WD40 domaincontaining proteins have 4 to 16 repeating units with the most being a 6 to 8 bladed beta propeller and each blade comprises a four-stranded anti-parallel b-sheet (figure 1A) (Li and Roberts, 2001; Smith et al., 1999; Stirnimann et al., 2010). WD40 repeats are known to act as a site for protein-protein interaction, serve as platforms for the assembly of protein complexes or mediators of transient interplay among other proteins. There are two known tandem WD40 repeats in the Frtz protein (Collier et al., 2005). However, when I re-analyzed the structure of Drosophila Frtz protein using WD40-repeat protein Structure Predictor (WDSP) (Wang et al., 2015), six WD40 repeats were predicted in the protein, which is four more repeats than previous data showed (Collier et al., 2005) (figure 1B). Similarly, seven WD40 repeats were predicted in the human Frtz (hFrtz) protein, containing five more repeats than the published data (figure 1 C). These data suggest the previous study showing only two WD40 repeats in both Drosophila 
Frtz and human Frtz protein may be inaccurate since most WD40 repeats containing proteins have more than four WD40 repeats.

WDSP predicted 7 hotspot residues with high potential for protein interaction with In, and two of them are located within the known Drosophila Frtz WD40 fragment. Because the known Frtz WD40 fragment has been demonstrated to lead to the strongest interaction, only these predicted hotspot residues were included for the mapping study. Also, since the great majority of protein-protein interaction interfaces reflect the composition of protein surfaces, rather than the protein cores (Stirnimann et al., 2010; Yan et al., 2008a), a few more residues were selected based on the predicted structure given by WDSP and structure features of WD40 domain reviewed by Stirnimann's and colleagues (Stirnimann et al., 2010). Furthermore, only the amino acids which are not conserved in both Frtz WD40 homologs across other species and other WD40 motif containing proteins were further selected. The conserved residues in Frtz WD40 across species and WD 40 containing proteins may play an important role in maintaining the overall structure of WD40 motif which we did not want to destroy. 12 amino acid residues were selected to be tested and these are listed in table 1.

All non-Alanine residues were changed to Alanine, and Alanine was changed to Glycine. All 12 mutations were introduced into a single Drosophila Frtz WD40 fragment. Surprisingly, this highly mutated fragment still interacted strongly with In in the Yeast-twohybrid system. We also tried only two amino acids mutations together, N366\&E367, but the mutations also didn't block the interaction with In.

\section{Generation of in precise deletion $\left(i n^{P D}\right)$ stock using CRIPSR/Cas9 system}

in, fy and frtz are planar polarity effector genes that share very similar mutant phenotype and interact strongly (Collier et al., 2005; Lee and Adler, 2002). These genes function 
downstream of PCP core genes such as $f z$, vang and $d s h$ (Wong and Adler, 1993), and in many cells in, fy or frtz mutants produces multiple hairs, usually 2-3 hairs in each wing cell and the polarity of the hairs are altered (Collier et al., 2005; Wong and Adler, 1993). Double mutants of any two of these 3 genes have the similar mutant phenotype as the single mutant and the genes show strong genetic interactions (Adler et al., 2004; Collier et al., 2005; Lee and Adler, 2002; Wang et al., 2014; Wong and Adler, 1993). For example, any double mutant combination of weak PPE alleles results in a strong PPE mutant phenotype (Collier et al., 2005).

In Chapter 2, I described that the mild overexpression of Frtz almost completely suppressed the mutant phenotype of a temperature sensitive hypomorphic allele of $i n$, $i n^{t s}$. We also found another in allele $i n^{I H 56}$, which results from a nonsense mutation, is also almost completely suppressed by mild overexpression of Frtz (Chapter 2 figure 7). However, neither of these in alleles are null mutations. Nonsense mutation may produce some protein by readthrough, and that protein may be stabilized by over-expressing Frtz (Wang et al. 2014).

In order to generate an in null mutation, I used the CRISPR/Cas 9 genome editing technique (Gratz et al., 2013a; Gratz et al., 2013b; Ran et al., 2013). I designed two guide RNAs (gRNA) specifically targeting the 5' and 3' ends of the in gene. Figure 2 shows the location of in gene in Drosophila genome and where the two designed gRNAs target. At the same time, I designed a homology directed recombination (HDR) template for repairing the gap introduced by the two gRNAs (figure $2 \mathrm{~A}$ ). The HDR template includes two $1.5 \mathrm{~kb}$ homology arms from both in gene flanking regions and a Ds-Red fluorescent sequences in between for screening the flies carrying the insert. Plasmids that would drive expression of the gRNAs and the HDR template were injected into Cas9 expressing embryos. From this manipulation, 30 larva survived and these were crossed to an in mutant. Four out of the $30 \mathrm{G} 0$ flies gave rise to in 
mutant G1 progeny, all of which were subsequently found to be the result of the designed precise deletion of the in gene $\left(i n^{P D}\right)$. One of the independent lines carried the HDR template and expressed Ds-Red. in $^{P D}$ shows a significantly stronger mutant phenotype than in ${ }^{I H 56}$ (figure $2 \mathrm{~B} \& \mathrm{C})$. I quantified the frequency of cells that formed multiple hairs in two regions of the wing and in both the $i n^{P D}$ wings had a stronger phenotype. The hair polarity phenotype also appeared somewhat stronger in $i n^{P D}$, but this phenotype was not quantified.

\section{Mild overexpression of $f r t z$ partially rescues in ${ }^{P D}$ mutant phenotype}

$i^{I H 56}$ carries a premature stop codon which leads to a truncated In protein. The newly generated $i n^{P D}$ stock shows a significant stronger mutant phenotype than $i{ }^{I H 56}$ which suggests in nonsense mutation produces some protein by read-through, and that protein is stabilized by over-expressed Frtz.

In order to test if mild overexpression of frtz can suppress the mutant phenotype of $i n^{P D}$, similarly to $\mathrm{in}^{\text {ts }}$ and $\mathrm{in}^{\mathrm{IH56}}$, apterous-Gal4 (ap-Gal4) was used to drive the expression of frtz only in the dorsal layer of the wing. The quantitative analysis was done using wing hairs from two

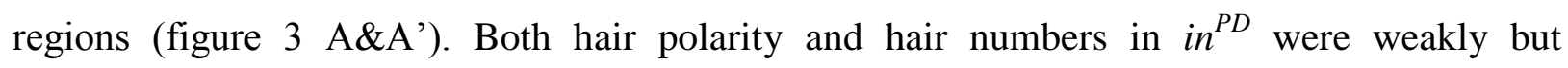
significantly rescued by mild overexpression of frtz (figure 3 B-D', E). This suggests that Frtz has In independent functions in PCP in Drosophila, or that at least it can function in PCP in the complete absence of In. Unlike the situation with $i n^{t s}$ and $i n^{I H 56}$, the $i n^{P D}$ mutant phenotype was only weakly rescued consistent with Frtz having In dependent functions in PCP, too.

\section{PPE proteins Fy and Frtz physically interact with upstream core protein Dsh}

In, Fy and Frtz proteins accumulate on proximal side of wing cells and function together in a protein complex (Wang et al., 2014). However, how PPE proteins or the PPE protein 
complex is recruited to the proximal edge of the cell still unclear. Our hypothesis is that PPE proteins are recruited asymmetrically by upstream PCP core proteins through direct physical interaction. The physical interaction could come from proximally localized proteins, Vang or Pk, or distally localized proteins Fz, Dsh or Dgo. I tested the interaction of Pk and Dsh with PPE proteins In, Fy and Frtz individually by yeast-two-hybrid system and coimmunoprecipitation assays. No evidence was obtained for a PPE protein physically interacting with Pk protein by the yeast-two-hybrid assay. However, I obtained clear evidence that Fy and Frtz interact with Dsh using both yeast-two-hybrid and co-immunoprecipitation assays (figure 4). Since the interaction between mammalian Dsh homolog Dvl2 and Fy homolog Fuz has been demonstrated in the published research (Zilber et al., 2013), a greater effort was made to understand the interaction between Frtz and Dsh.

In order to understand what fragment in Frtz is responsible for the interaction with Dsh, several Frtz fragments were generated (figure 5A), but only full length Frtz interacted with Dsh, suggesting the interaction comes from multiple locations all of which are required or that the protein as a whole is needed to fold into a conformation that allows the interaction with Dsh. In order to test this hypothesis, I generated a set of Frtz fragments where 100 amino acid residues at a time (300 base pairs) were deleted from full length Frtz (figure 5B). Each plasmid was tested by yeast-two-hybrid and screened for a loss of interaction with Dsh.

Yeast-two-hybrid data shows only the truncated Frtz proteins, which had amino acids $300-400,400-500,500-600,600-700$ or 800-951 deleted, interacted with Dsh, suggesting these five fragments aren't important for the interaction. It also suggests the rest of Frtz protein, amino acids 1-300 and 700-800 are required for this interaction.

\section{Protein interactions between mammalian PPE homologs}


In chapter 2, I described the physical interaction between two PPE proteins, In and Frtz. Also, an interaction between Frtz and Dsh was found by the yeast-two-hybrid and coimmunoprecipitation assay described earlier in this chapter. In order to determine if these interactions are conserved in their mammalian homologs, human intu (in homolog), wdpcp (frtz homolog) and $d v l 2$ (one of the three $d s h$ holomogs) were sub-cloned into yeast-two-hybrid vectors and the protein interaction assays were performed.

There are three isoforms of $d v l(d v l l, d v l 2$ and $d v l 3)$ with specific as well as overlapping functions in mammals. The isoforms also show a high degree of conservation (Etheridge et al., 2008; Hamblet et al., 2002; Rodriguez-Gil et al., 2013). All Dvls are cytoplasmic protein containing an amino-terminal DIX domain, a central PDZ domain, and a carboxyl-terminal DEP domain (Semenov and Snyder, 1997; Sussman et al., 1994). These conserved domains most likely mediate protein-protein interactions with other PCP proteins (Kim and Sheng, 2004; Wong et al., 2003). Dvl2 was found essential for normal cardiac morphogenesis, somite segmentation, and neural tube closure (Hamblet et al., 2002). Research showed $d v l 2$ interacts with fy mammalian homolog fuz to regulate ciliogenesis (Zilber et al., 2013), suggesting it may also interact with other mammalian PPE proteins. WDPCP protein is the mammalian homolog of Frtz. Similarly, there are two tandem WD40 repeats in WDPCP coding region (Collier et al., 2005). Human Intu protein contains a PDZ domain, and the location is similar to its Drosophila In homolog (Cho et al., 1992; Nagase et al., 1999; Te Velthuis et al., 2007).

I found a positive interaction between Intu and both Dvl2 and WDPCP (figure 6A and B). The interaction between Drosophila In and Frtz was demonstrated in previous study (Wang et al., 2014), suggesting that it may also conserved in other species. The interaction between 
Frtz and Dsh in Drosophila was not detected in its mammalian homologs Dvl2 and WDPCP. But it is possible that WDPCP interacts with other Dvl isoforms, such as Dvl3. 


\section{Discussion}

\section{Improvement of Frtz WD40 mapping assay}

The 12 mutations we tested (table 1) for studying the interaction with In are all predicted to be on the surface of the Frtz protein. Hence, our expectation was that some of these residues were likely to contribute to the interaction with the In protein (Stirnimann et al., 2010). However, our data did not provide support for this hypothesis. There are a variety of possible reasons for this. One is that the WDSP software incorrectly predicted the structure of this WD40 propeller. It is also possible that this interaction uses WD40 amino acids from a part of the propeller that is not typical of what have been seen in published interactions. We also tested the

$\mathrm{frtz}^{3}$ mutant, a temperature sensitive hypomorphic allele and it didn't block the interaction with In, too. Given our unexpected results I do not think it would be profitable to continue this line of research.

\section{Functions of Frtz}

The mild overexpression of Frtz partially rescued in ${ }^{P D}$ (figure 3), indicating that Frtz has some PCP activity in the complete absence of In. This could be due to Frtz having In independent function. An alternative hypothesis is that an important function of In in PCP is to stabilize Frtz and that the mild overexpression allowed some Frtz to accumulate even in the absence of any In. Consistent with this hypothesis is that the gain of function phenotype associated with the high level over expression of Frtz was not blocked in an in mutant (Wang, 2014). It is also possible that Frtz may also function as an effector in another PCP pathway, such as the dachsous $(d s) / f a t(f t)$ pathway. Although several group reported that $d s / f t$ pathway functions upstream of $f z /$ stan pathway, such as in Drosophila compound eye (Yang et al., 2002), in other tissues may not be the truth. Research showed $d s / f t$ and $f z / s t a n$ pathways function in 
parallel in abdomen (Casal et al., 2006). Double mutants of one gene in $d s / f t$ pathway and one gene in $f z / s t a n$ pathway showed a more severe phenotype than any single mutant. In Drosophila wing, $d s / f t$ and $f z / s t a n$ pathways may also function in parallel and Frtz maybe the effector of both pathway.

\section{Antagonistic force between Dsh and PPE proteins Fy and Frtz}

Our yeast-two-hybrid data showed there are direct interactions between Fy and Dsh, Frtz and Dsh. However, we did not find convincing evidence for a direct interaction between proximally localized Pk and PPE proteins. Dsh asymmetrically accumulates on the distal side of wing cell, which is opposite to the side where Frtz and Fy expressed. The antagonistic forces between Dsh and Fy or Frtz most likely contribute to asymmetrical accumulation of PPE protein or protein complex. It is also possible that the positive recruitment by proximal PCP core protein also contributes to the asymmetric accumulation of PPE proteins, but the positive recruitment is not coming solely from $\mathrm{Pk}$. It is possible that Vang or a combination of $\mathrm{Pk}$ and Vang functions to recruit the PPE proteins.

\section{Interaction between Frtz and Dsh}

Our yeast-two-hybrid data showed the proximally localized protein Frtz interacted with distally localized protein Dsh. Multiple locations in Frtz (Figure 5) are required for this interaction. Six WD40 (WD40-1 to WD40-6) repeats in Drosophila Frtz were predicted using WDSP, and the two known WD40 repeats (Collier et al., 2005) belong to WD40-4 and WD40-5 repeats which are not required for the interaction with Dsh suggested by yeast-two-hybrid data (figure 5). However, the predicted WD40 repeats WD40-1 to WD40-3 are responsible for this interaction. 
There is no known structural motif found in Frtz amino acid 700-800, but it may help protein folding or stabilizing the protein, since we previously observed the increased expression of Dsh when Frtz was over expressed (Wang et al., 2014).

\section{Interactions between mammalian PCP core protein and PPE protein homologs}

We found human Intu (Drosophila In homolog) interacted with human WDPCP protein (Drosophila Frtz homolog) using yeast-two-hybrid system. In chapter 2, I described the interaction between In and Frtz demonstrated by yeast-two-hybrid and co-immunoprecipitation assay. Together, data suggest the interaction between In and Frtz are well conserved. The interaction between Frtz and Dsh in Drosophila hasn't been detected in mammalian homologs. Yeast-two-hybrid data showed there is no direct interaction between WDPCP and Dvl2. However, there is a possibility that WDPCP might interacts with other Dvl isoforms, such as Dvl3. Mammalian Dvl isoforms have specific but overlapping functions. They also show a high degree of conservation. Research showed a mild PCP phenotype was observed in $d v l 3^{-/-}$ cochleae, where the uniform orientation of stereociliary bundles was disrupted in some hair cells in both the base and the middle of the cochlear ducts. The severity of the phenotype was much increased in $d v l 3^{-/-} ; v^{2} g^{L t a p L p /+}$ mutants that showed neural tube defects, in which many hair cells had disrupted orientation (Etheridge et al., 2008). Thus, Dvl3 is a good candidate to be tested for interaction with WDPCP. 
Figure 1. Topology of both Drosophila and human Frtz WD40 repeats predicted by WDSP.

(A) General structure of WD40 repeats from (i) side and (ii) top view. Only the 7-bladed beta propeller is shown here. (iii) Side view of each beta propeller blade consisting D,A,B,C fourstranded anti-parallel b-sheet. Residues which are often involved in peptide interactions on the top surface are highlighted with red asterisks. (B)WDSP predicted topology of Drosophila Frtz WD40 repeats. The hotspot residues are highlighted with red. (C) WDSP predicted topology of human Frtz WD40 repeats. The hotspot residues are highlighted with red. 
A
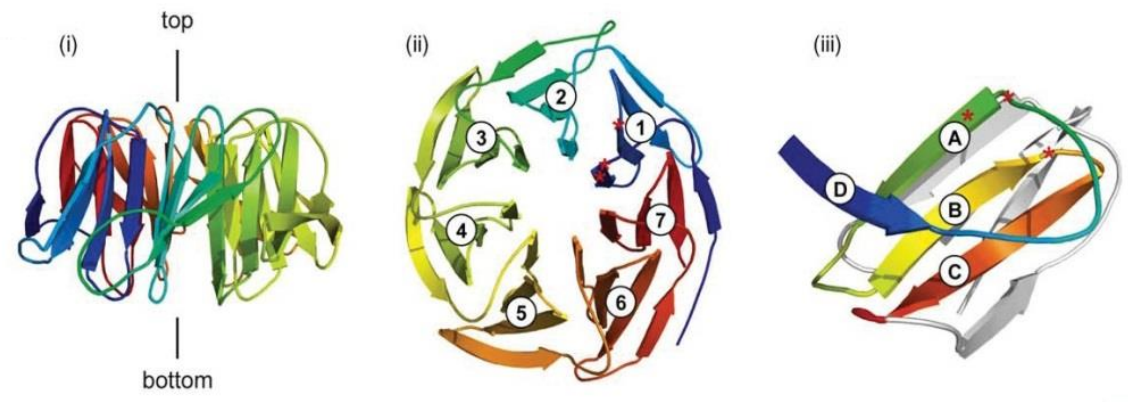

Stirnimann et al., 201

\section{B}

Drosophila Frtz is a WD40 protein. The average score is :53.17

The estimated Q3 accuracy is: $86+5 \%$

Topology of Drosophila_Frtz

\begin{tabular}{|c|c|c|c|c|c|c|c|c|c|c|c|}
\hline Repeats & Score & $\begin{array}{l}\text { Start } \\
\text { End }\end{array}$ & $\begin{array}{c}\text { Strand } \\
\quad \mathrm{d}\end{array}$ & $\begin{array}{l}\text { Loop } \\
-\mathrm{da}\end{array}$ & $\begin{array}{c}\text { Strand } \\
-^{\mathrm{a}}\end{array}$ & Loop_ab & $\begin{array}{c}\text { Strand } \\
\text { - b }\end{array}$ & Loop_bc & $\begin{array}{c}\text { Strand } \\
-\mathrm{C}\end{array}$ & $\begin{array}{l}\text { Løop } \\
\text { _cd }\end{array}$ & $\begin{array}{l}\text { Hotspots } \\
\text { on the top }\end{array}$ \\
\hline WD1 & 52.07 & $123-167$ & VFEKYL & VGKLASEV & ITDAFF & TRS & HIVLAY & NTN & QLTVVH & LQRPNAR & NA \\
\hline WD2 & 20.55 & $168-242$ & SQGPEK & IANMDPR & IFHVII & PGATERKLSRHLTVNASFD & LFVVWT & QSSQNEVYPWRPTIRDQDRA & NIHVFK & IKGLQ & R180 F182 \\
\hline WD3 & 33.51 & 243-295 & LESIAY & CWSEND & PLCVDF & LRSSES & QIITLE & QKVSRKGDISA & EICSYE & LAAGKM & D254 L256 \\
\hline WD4 & 71.81 & 296-339 & QRTAIT & SIPMGAQ & ICSFAF & SPDQE & KLFLGS & VDR & NICLHD & LVQQS & Q308 \\
\hline WD5 & 51.88 & $340-382$ & TKYANQ & IEIV & PNQCAW & HCDSA & MLCVAN & ERS & VLQCFD & LALATIG & N351 \\
\hline WD6 & 40.12 & $383-423$ & HQLVSE & NVTPSS & LLDLSH & YFVAQP & TLLSVA & FSRKP & DLSTFK & & L396 \\
\hline
\end{tabular}

C

Human Frtz is a WD40 protein. The average score is :46.05

The estimated Q3 accuracy is: $85+5 \%$

Topology of Human_Frtz

\begin{tabular}{|c|c|c|c|c|c|c|c|c|c|c|c|}
\hline Repeats & Score & $\frac{\text { Start }}{\text { End }}$ & $\begin{array}{c}\text { Strand } \\
\text { d }\end{array}$ & $\begin{array}{l}\text { Loop } \\
\text { _da }\end{array}$ & $\begin{array}{c}\text { Strand } \\
{ }_{-}{ }^{a}\end{array}$ & Loop_ab & $\begin{array}{c}\text { Strand } \\
\text { - } b\end{array}$ & $\begin{array}{l}\text { Loop } \\
\text { _bc }\end{array}$ & $\begin{array}{c}\text { Strand } \\
{ }_{-}{ }^{c}\end{array}$ & Loop_cd & $\begin{array}{l}\text { Hotspots } \\
\text { on the top }\end{array}$ \\
\hline WD1 & 53.61 & $115-153$ & RCVLSK & WKNKY & VCQLLF & GSG & VLVSLS & LSGP & QLEKW & IDR & Y125 \\
\hline WD2 & 40.79 & 154-196 & SLVGKL & ISDT & ISDALL & TDSF & IILSFL & AQN & KLCFIQ & FTKKMESS & NA \\
\hline WD3 & 23.39 & $197-252$ & DVNKRL & EKLSALD & YKIFYY & EIPGPINKTTER & HLAINC & VHDR & VVCWWP & LVNDDAWPW & D209K211 \\
\hline WD4 & 30.96 & 253-326 & APISSE & KDRAN & LLLLGY & AQGRL & EVLSSV & RTEWDPLDVRFGTKQPY & QVFTVE & HSVSVDKEPMADSCIYECIRNKI & N263 L265 \\
\hline WD5 & 69.04 & $327-370$ & QCVSVT & RIPLKSKA & ISCCRN & VTED & KLILGC & EDS & SLILYE & THRRV & E357 \\
\hline WD6 & 60.22 & $371-424$ & TLLAQT & ELLPS & LISCHP & SGA & ILLVGS & NQG & ELQIFD & MALSPINIQLLAEDRLPRE & 1383 \\
\hline WD7 & 35.67 & $425-477$ & TLQFSK & LFDASSS & LVQMQW & IAPQVVSOKGEGSDIYD & LLFLRF & ERGPL & GVLLFK & & NA \\
\hline
\end{tabular}


Figure 2. $i{ }^{\mathrm{PD}}$ design scheme and phenotype quantification. (A) in genomic location, gRNA and HDR template design. (i). The in gene is located on the 3rd chromosome at nucleotide $20,367,198$ to $20,370,259$. The squence targeted by the two gRNAs on the genome is highlighted by a red X. (ii) HDR template design. two $1.5 \mathrm{~kb}$ homolog arms were amplified from in flankng regions on both end. Ds-Red fluorescent sequence was inserted between two homolog arms which can be further removed by lox/cre recombination. The gene region between two dash line in (i) is replace by sequence in (ii) after homolog directed repair. ( B ) \& ( C ) are $i n^{\mathrm{IH} 56}$ and $i n^{\mathrm{PD}}$ phenotype quantitation. Two regions from the Drosophila wing are shown. The number of wing cells forming multiple hairs in $i n^{\mathrm{PD}}$ is significantly greater than in $i n^{\mathrm{IH} 56}(\mathrm{p}<0.001)(\mathrm{n}=20)$. (D) \& (E) show wing hair phenotype from two regions of $i n^{\mathrm{IH} 56}$. (F) \& (G) show wing hair phenotype from the same two regions in $i n^{\mathrm{PD}}$. 
A

(i)

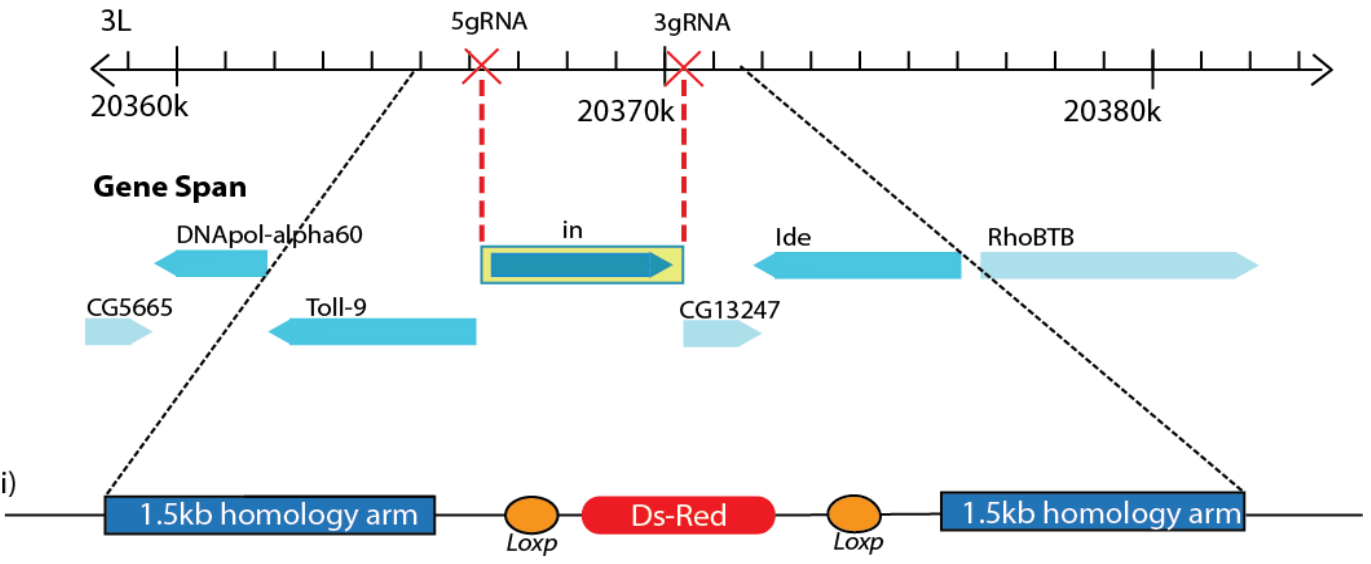

B

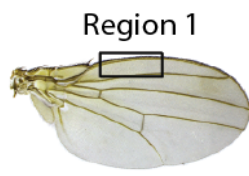

$\%$ of cells forming multiples hairs

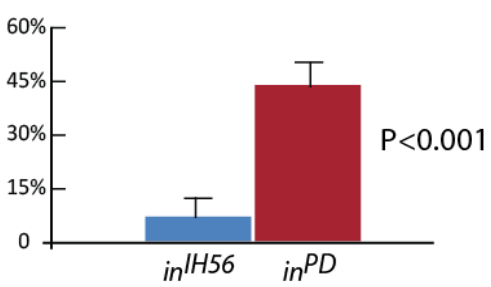

C

Region 2

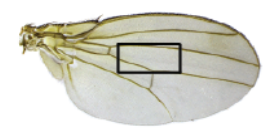

$\%$ of cells forming multiples hairs

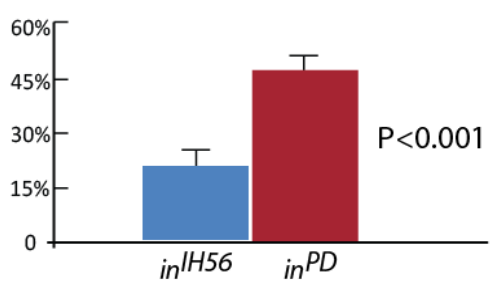

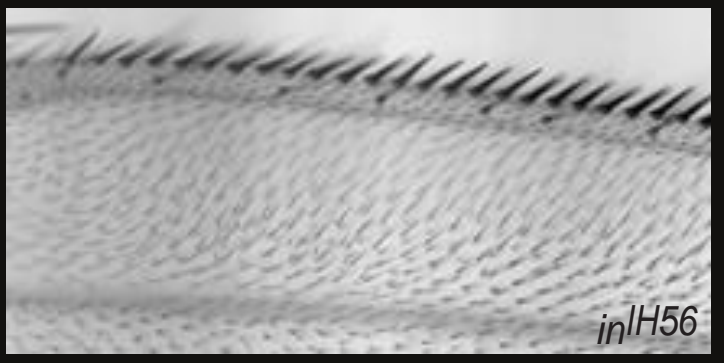
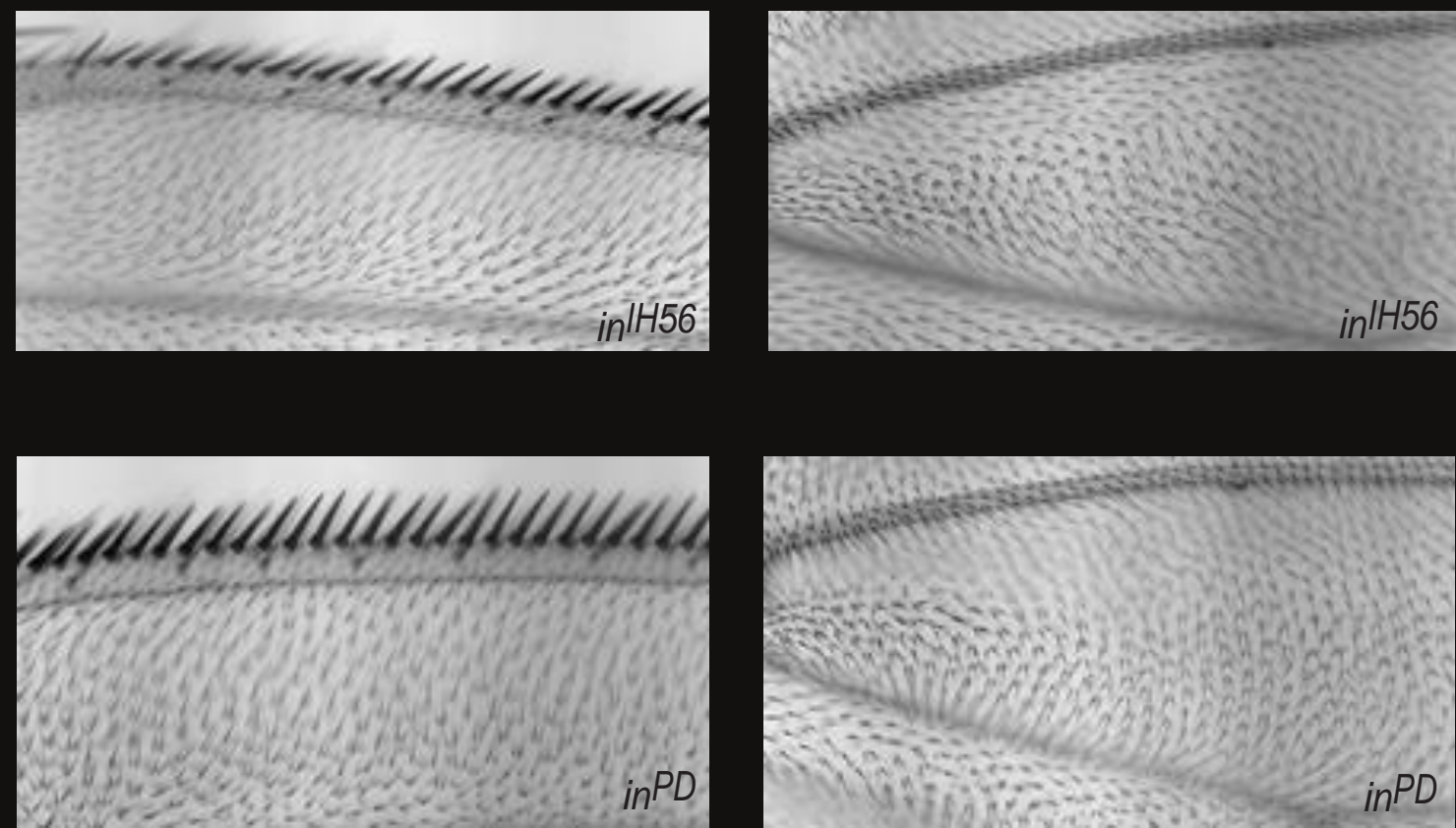
Figure 3. The Mild overexpression of myc-frtz partially rescues i ${ }^{\mathrm{PD}}$. A and A' show Drosophila wings. Two regions where analysis were done are marked by black rectangels. B and B' show wing hair phenotype using $p$ WALIUM-myc-frtz/apGAL4; in ${ }^{\mathrm{PD}}$ wing. $\mathrm{C}$ and $\mathrm{C}^{\prime}$ show wing hair phenotype using $p$ WALIUM-myc-frtzl $+; i^{\mathrm{PD}}$ wing. $\mathrm{D}$ and $\mathrm{D}^{\prime}$ show $i{ }^{\mathrm{PD}}$ wing hair phenotype. $\mathrm{E}$ shows $i{ }^{\mathrm{PD}}$ quantative analysis with or without overexpression of myc-frtz. The difference is significant $(n=20, p<0.001)$ 
Figure 3

A

Region 1
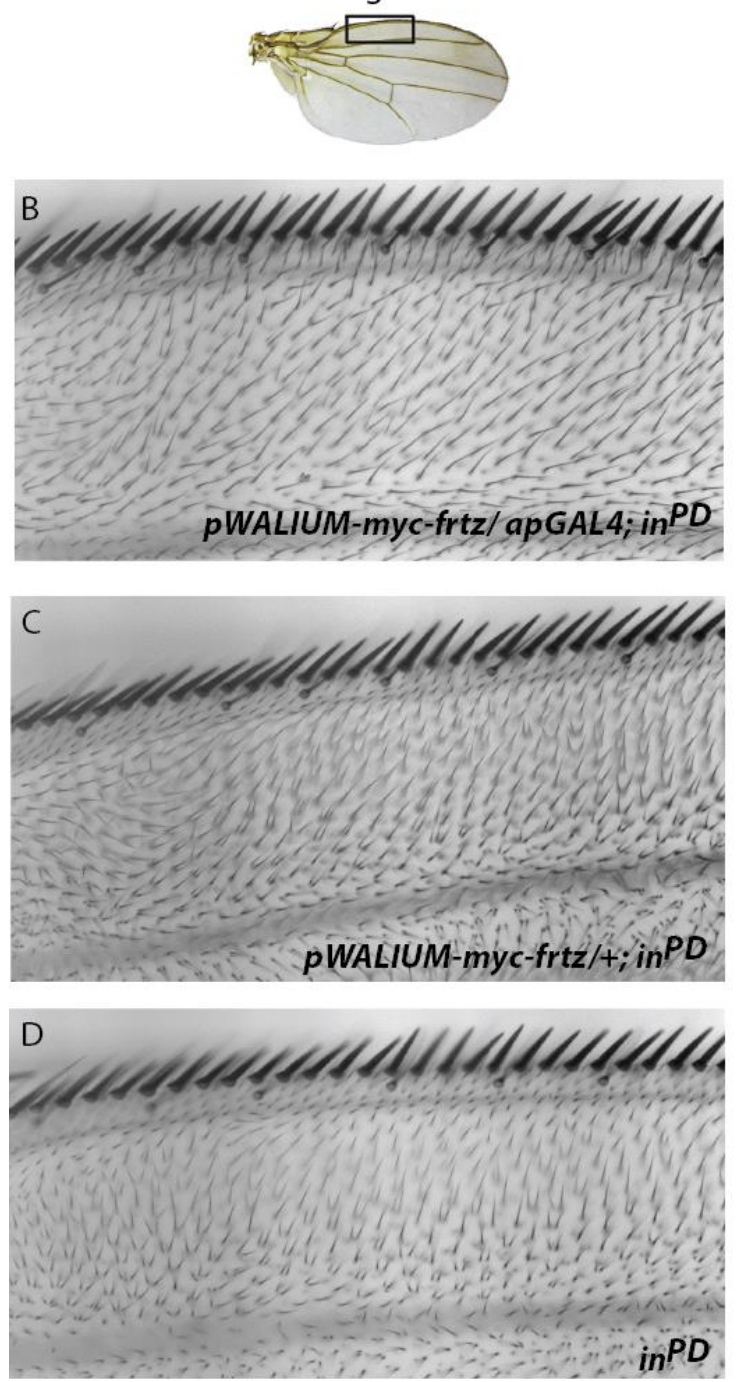

$A^{\prime}$

Region 2
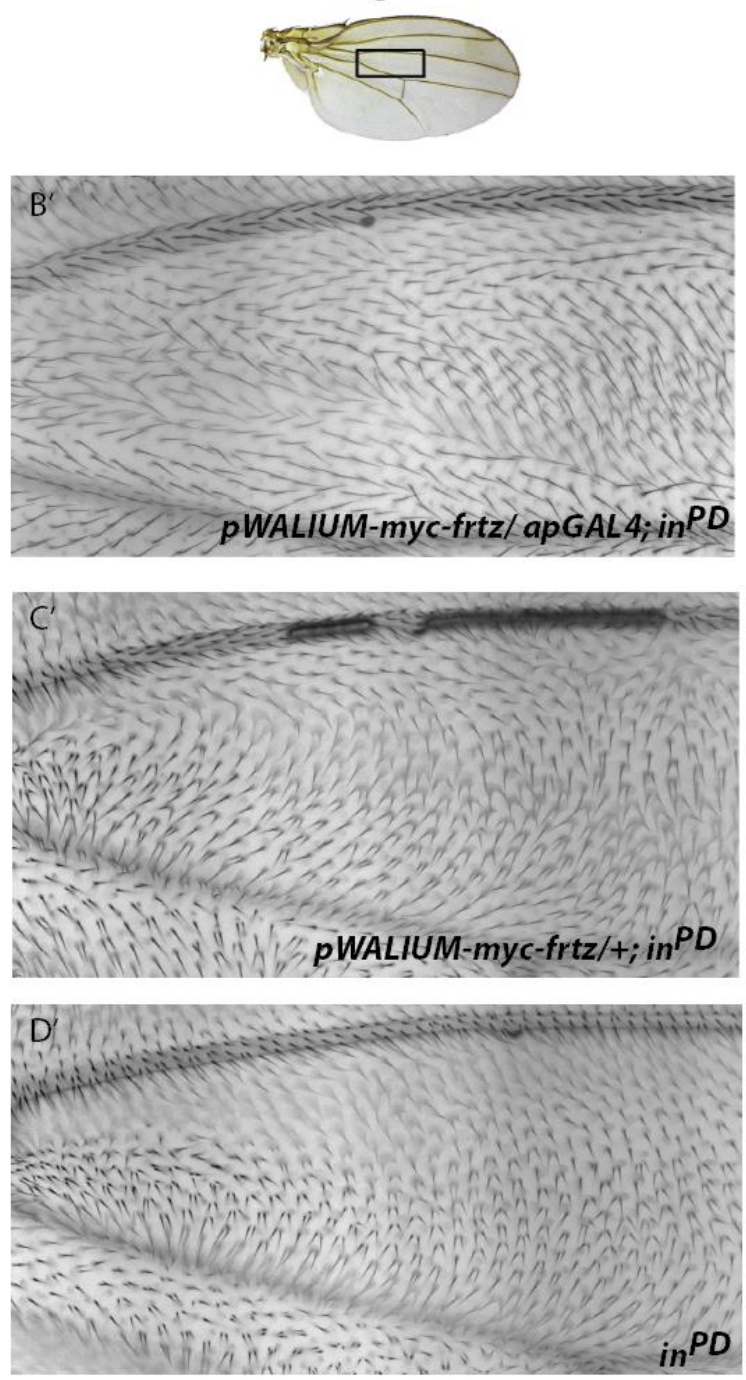

\section{E}

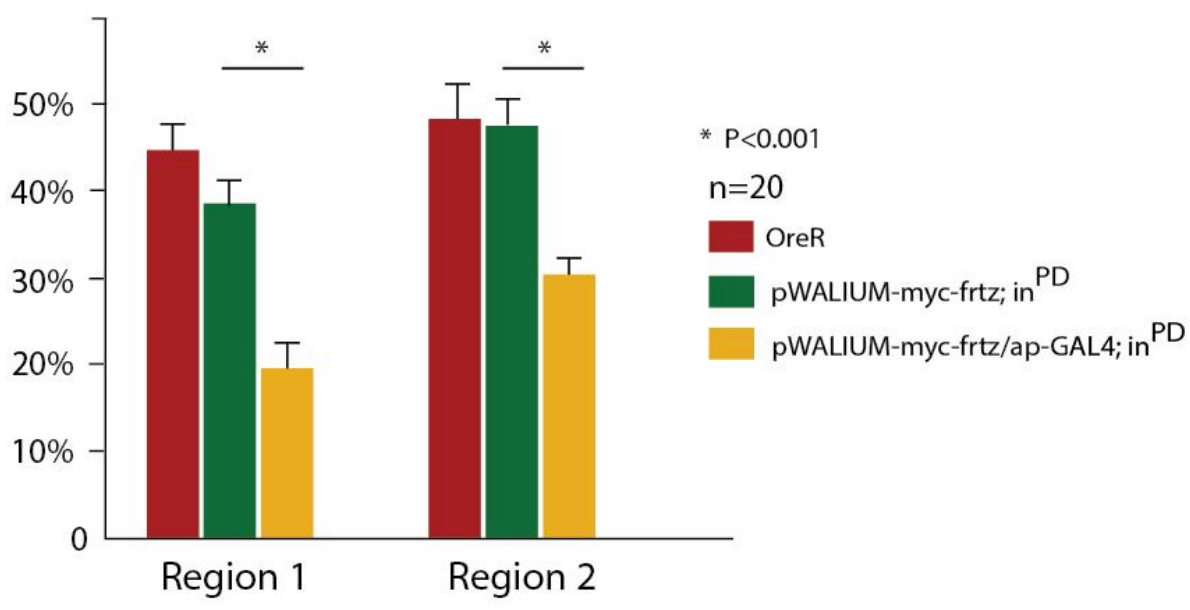


Figure 4. Protein interactions between PPE and PCP core proteins. (A). A summary of the interactions between PPE and PCP core proteins. + indicates there is physical interaction and indicates no direct interaction was detected by the yeast-two-hybrid system. (B) and (D) are yeast-two-hybrid plates for interaction between Fy and Dsh (B), Frtz and Dsh (D). The plates are 4 amino acid (Ade/-His/-Leu/-Trp) drop out plates containing $\mathrm{x}$ - $\alpha$-gal . Only the colonies with interacting protein pairs grow and turn blue. (C) and (E) are co-immunoprecipitation results for Fy and Dsh (C) Frtz and Dsh (E), respectively. (C) shows the coimmunoprecipitation of Dsh and Fy. This experiment used UAS-Fy-GFP driven by ptc-Gal4 and Dsh-myc transgenes (Bloomington Drosophila Stock Center stock number 25385). Wing disc samples were immunoprecipitated using rabbit GFP antibody then detected with mouse GFP and Myc antibody for western blotting. Arrows point to Fy-GFP and Dsh-Myc on the Western blots. (E) shows the co-immunoprecipitation of Dsh and Frtz. This experiment used $U A S$-frtz driven by ptc-Gal4 and Dsh-myc transgenes. Wing disc samples were immunoprecipitated using rabbit Myc antibody then detected with rat Frtz and mouse Myc antibody for western bloting. Arrows point to Dsh-Myc and Frtz on the Western blots. 
A

\section{\begin{tabular}{l|l} 
Dsh & Pk
\end{tabular}}

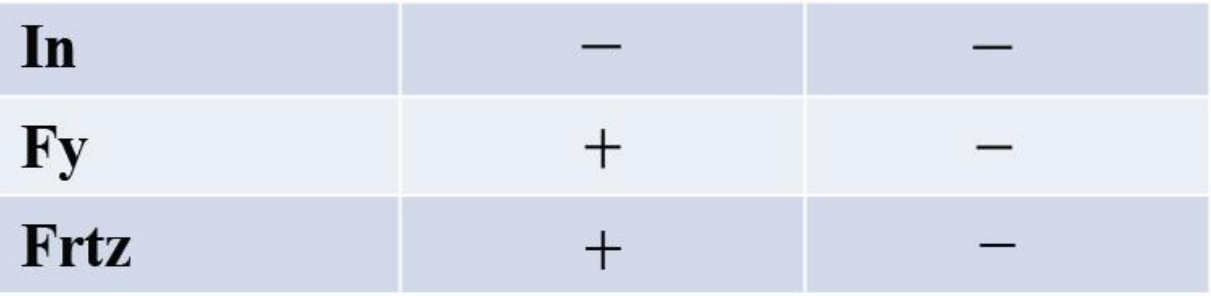

B

C WB w/GFP ab WB w/Myc ab

1. pGADT7-Fy+pGBKT7-Dsh

2. pGADT7-Fy+pGBKT7

3. $\mathrm{pGADT7+pGBKT7-Dsh}$

4. pGADT7+pGBKT7

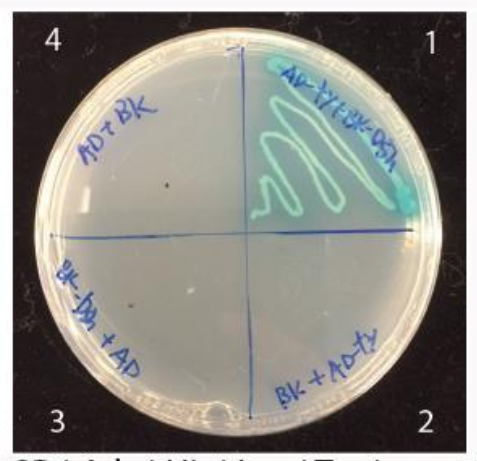

SD/-Ade/-His/-Leu/-Trp/x-a-gal

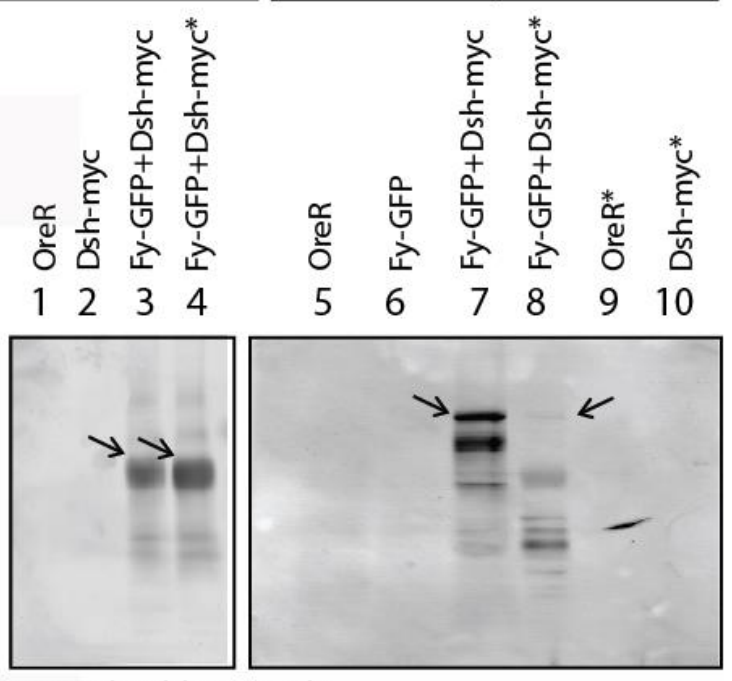

* IP with rabbit GFP ab

E

1. pGADT7-Dsh+pGBKT7-Frtz

2. pGADT7-Dsh+pGBKT7

3. pGADT7+pGBKT7-Frtz

4. pGADT7+pGBKT7

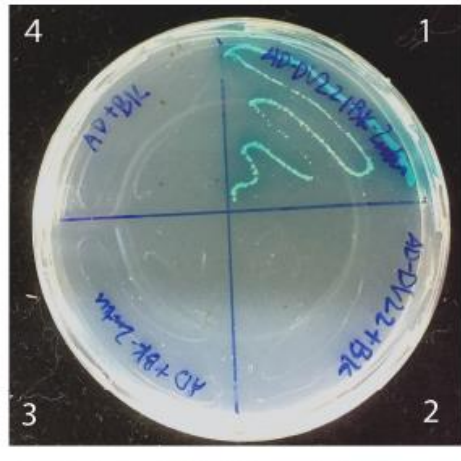

SD/-Ade/-His/-Leu/-Trp/x-a-gal

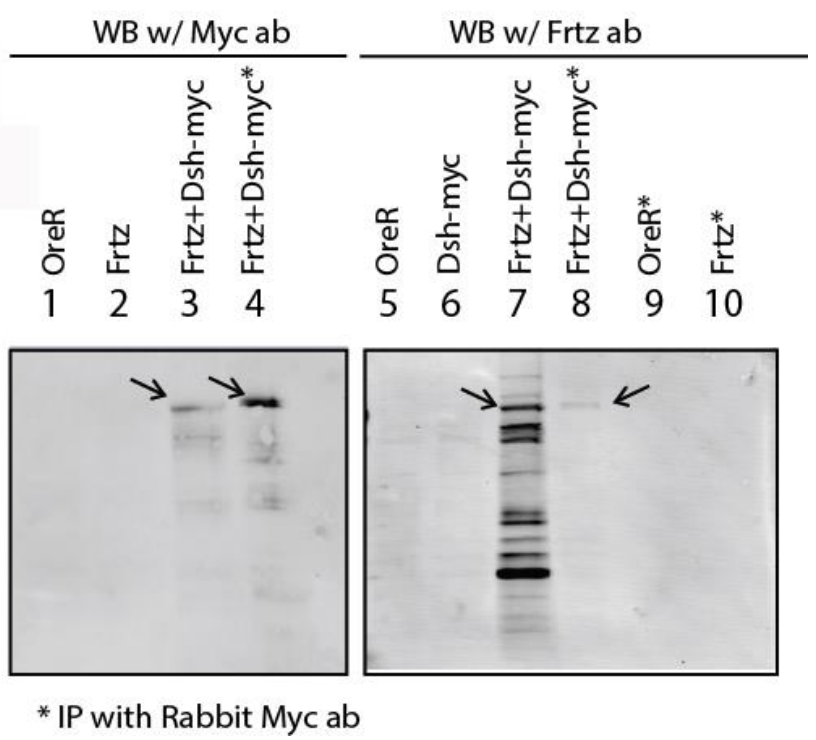


Figure 5. Fragments in Frtz responsible for the interation with Dsh. (A). Frtz fragment test. Various fragments of Frtz were tested for interating with Dsh. Only the full length Frtz interacted with Dsh. (B) Frtz Fragment deletion assay. Nine truncated forms of the Frtz gene were generated. A 300bp fragment (which encodes 100 amino acids) was deleted from full length frtz in each construct, except the last one which has a larger (151 amino acids) deletion. There is a Myc tag between Frtz fragments in the same construct that serves as a spacer. "+ " indicates there is interaction and " - " means there is no interaction detected using the yeasttwo-hybrid system. 
A

WD40

Interaction

Full length Frtz 1-951 a.a

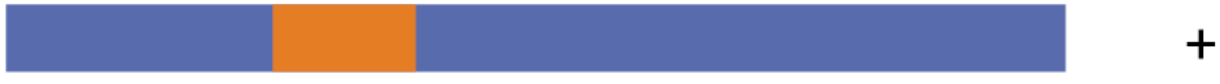

Frtz 1-400 a.a

Frtz 401-951 a.a

Frtz 200-400 a.a

Frtz $100-700$ a.a

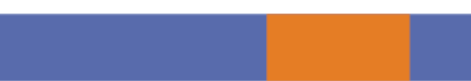

$+$

Frtz 200-600 a.a

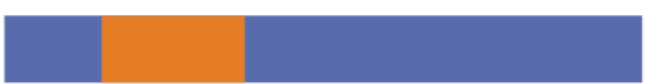

Frtz 300-500 a.a

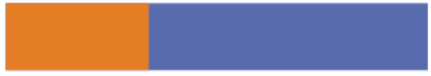

Frtz WD40 fragment

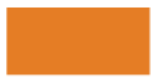

B

Frtz 100-951 a.a

Frtz 100-200 deleted

Frtz 200-300 deleted

Frtz 300-400 deleted

Frtz 400-500 deleted

Frtz 500-600 deleted

Frtz 600-700 deleted

Frtz 700-800 deleted
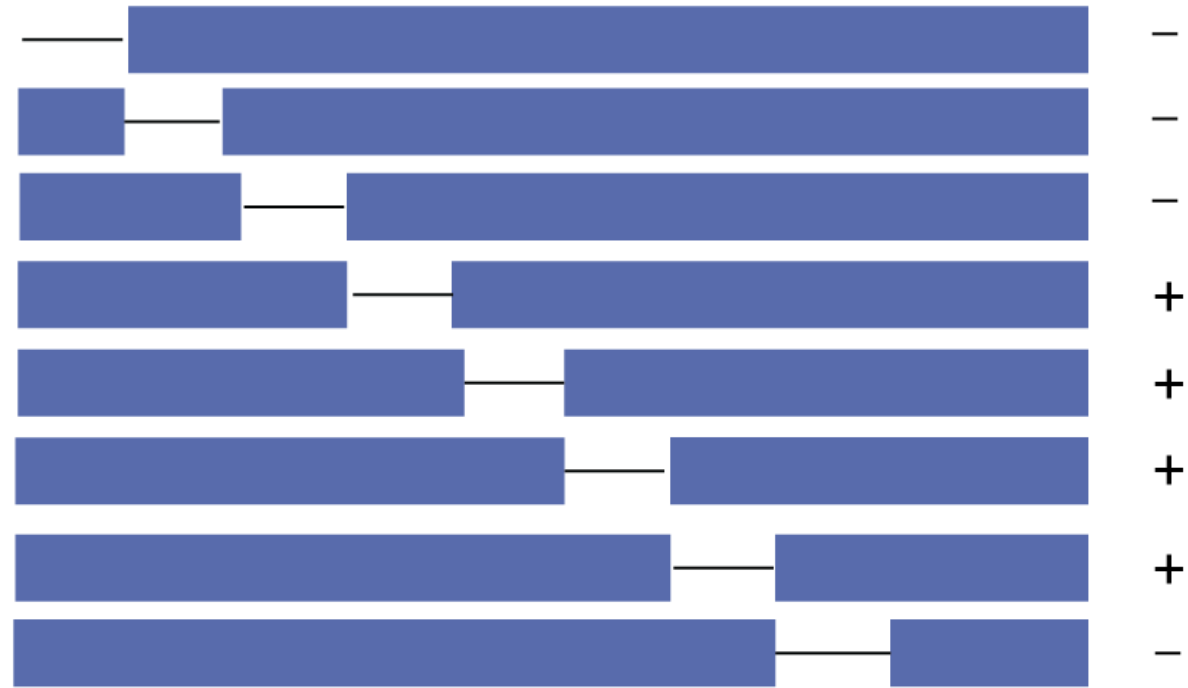

Frtz 800-951 deleted

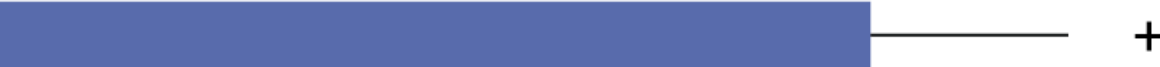


Figure 6. Yeast-two-hybrid plates for interaction between full length Dsh and various fragments of Frtz. Plasmids were co-tranformed into AH109 yeast cells, and then grow on low stringency plates with two amino acids drop out SD/-Leu/-Trp (A). Colonies from previous dropout medium were transferred to a four-marker dropout medium (SD/-Ade/-His/-Leu/-Trp) with Xalpha-Gal (B). Colonies that grew and turned blue were scored as showing a positive interaction. 


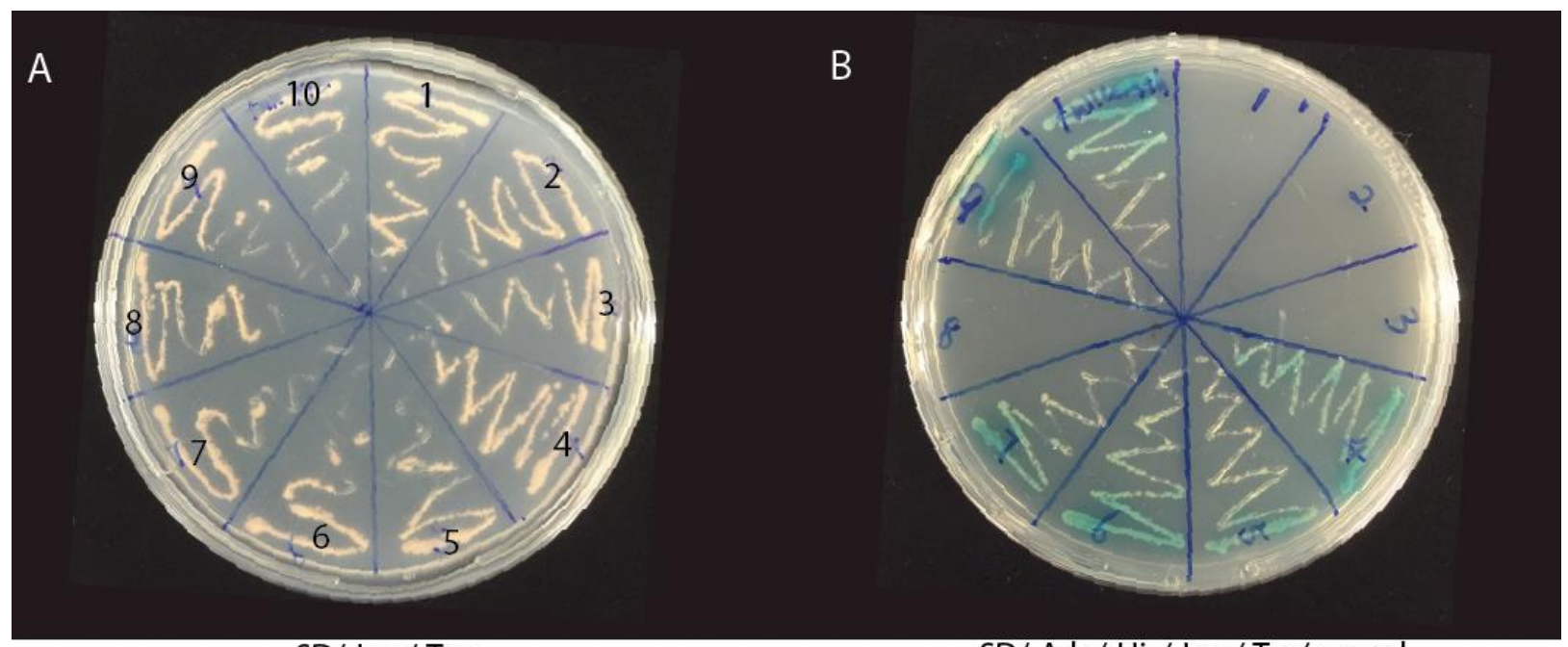

SD/-Leu/-Trp

SD/-Ade/-His/-Leu/-Trp/x-a-gal

1. pGADT7-Dsh+pGBKT7-Frtz 100-951aa

2. pGADT7-Dsh+pGBKT7-Frtz 1-100 \& 201-951aa

3. pGADT7-Dsh+pGBKT7-Frtz 1-200 \& 301-951aa

4. pGADT7-Dsh+pGBKT7-Frtz 1-300 \& 401-951aa

5. pGADT7-Dsh+pGBKT7-Frtz 1-400 \& 501-951 aa

6. pGADT7-Dsh+pGBKT7-Frtz 1-500 \& 601-951 aa

7. pGADT7-Dsh+pGBKT7-Frtz 1-600 \& 701-951aa

8. pGADT7-Dsh+pGBKT7-Frtz 1-700 \& 801-951aa

9. pGADT7-Dsh+pGBKT7-Frtz 1-800 aa

10. pGADT7-Dsh+pGBKT7-Frtz full length 
Figure 7. Yeast-two-hybrid plates for testing the interaction between Intu and Dvl2 (A), WDPCP and Intu (B). Plasmids were co-tranformed into AH109 yeast cell then grew on the high stringency plates with four amino acid drop out (Ade/-His/-Leu/-Trp). The plates also contained X-alpha-Gal. Colonies that grew and turned blue were scored as showing a positive interaction. 


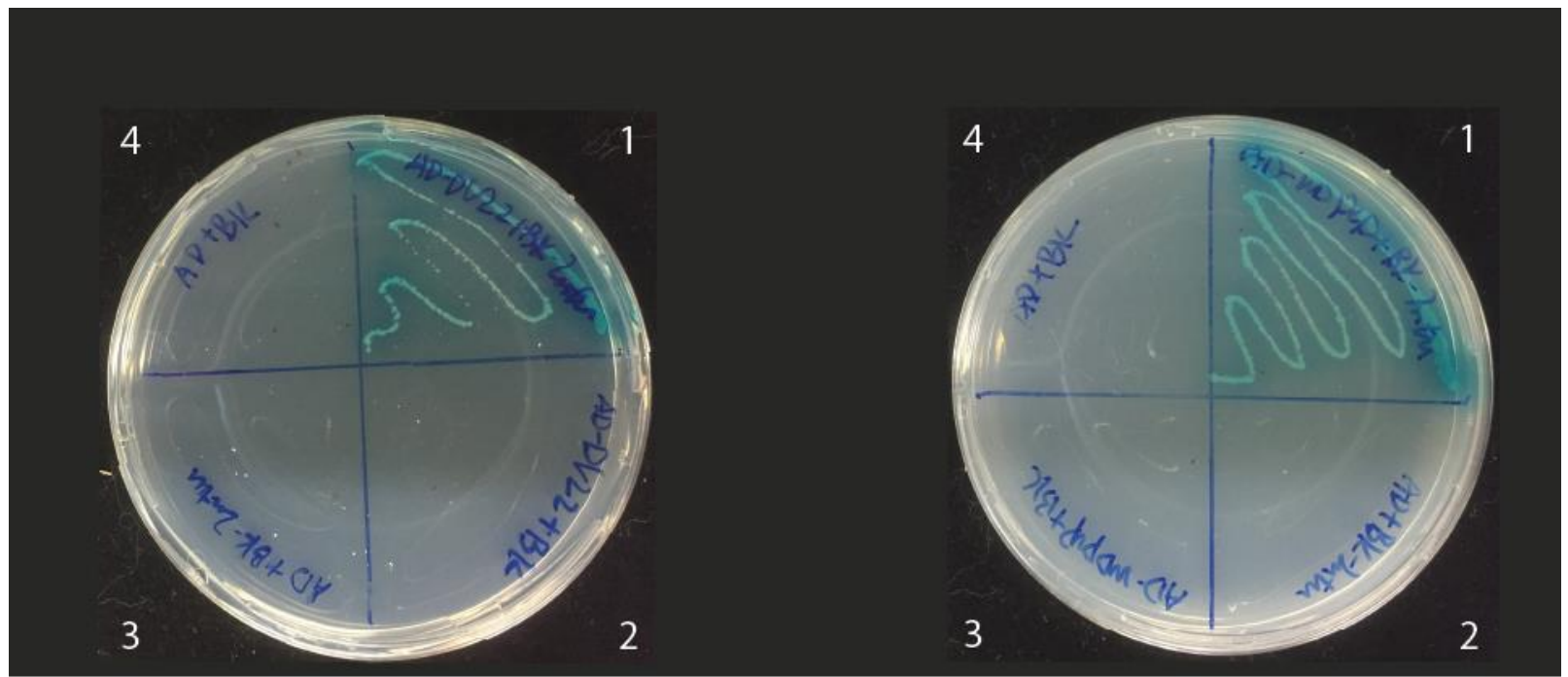

SD/-Ade/-His/-Leu/-Trp/x-a-gal

SD/-Ade/-His/-Leu/-Trp/x-a-gal

1. pGADT7-Dvl2+pGBKT7-Intu

1. pGADT7-WDPCP+pGBKT7-Intu

2. pGADT7-Dvl2+pGBKT7

2. pGADT7+pGBKT7-Intu

3. pGADT7+pGBKT7-Intu

3. pGADT7-WDPCP+pGBKT7

4. pGADT7+pGBKT7

4. $p G A D T 7+p G B K T 7$ 
Table 1. Amino acids in Frtz WD40-4 and WD40-5 repeats predicted as candidates for being responsible for the interaction with In. Only the potential interacting residues belonging to known WD40 fragments are included here. Four criteria are considered, (i) whether the residues are hotspot predicted by WDSP; (ii) whether the residues are conserved in Frtz WD40 proteins across other species, human, mouse, rat, xenopus; (iii) whether the residues are conserved in other WD40 containing proteins and (iv) whether the residues are on the protein surface, including top, bothom or side surface. Note, if the residues are conserved in Frtz WD40 but not other WD40 containing proteins ( or vise versa), those residues are considered appropriate here. 
Table 1.

\begin{tabular}{c|c|c|c|c}
\hline Candidates & $\begin{array}{c}\text { Hotspot } \\
\text { (predicted by WDSP) }\end{array}$ & $\begin{array}{c}\text { Conserved in } \\
\text { Frtz WD40 }\end{array}$ & $\begin{array}{c}\text { Conserved in } \\
\text { other WD40 proteins }\end{array}$ & On protein surface \\
\hline Q308 & Yes & No & No & Yes \\
\hline C310 & No & No & No & Yes \\
\hline V326 & No & No & Yes & Yes \\
\hline T340 & No & Yes & No & Yes \\
\hline A343 & No & Yes & No & Yes \\
\hline Q345 & No & Yes & No & Yes \\
\hline E347 & No & Yes & No & Yes \\
\hline N351 & Yes & No & Yes & Yes \\
\hline N366 & No & No & No & Yes \\
\hline E367 & No & No & Yes & Yes \\
\hline A377 & No & Yes & No & Yes \\
\hline Q384 & No & Yos & & \\
\hline
\end{tabular}




\section{Reference}

Adler, P.N. (2012). The frizzled/stan pathway and planar cell polarity in the Drosophila wing. Curr Top Dev Biol 101, 1-31.

Adler, P.N., Charlton, J., and Liu, J. (1998). Mutations in the cadherin superfamily member gene dachsous cause a tissue polarity phenotype by altering frizzled signaling. Development $125,959-968$.

Adler, P.N., Zhu, C., and Stone, D. (2004). Inturned localizes to the proximal side of wing cells under the instruction of upstream planar polarity proteins. Curr Biol 14, 2046-2051.

Amonlirdviman, K., Khare, N.A., Tree, D.R., Chen, W.S., Axelrod, J.D., and Tomlin, C.J. (2005). Mathematical modeling of planar cell polarity to understand domineering nonautonomy. Science 307, 423-426.

Axelrod, J.D. (2001). Unipolar membrane association of Dishevelled mediates Frizzled planar cell polarity signaling. Genes Dev 15, 1182-1187.

Bastock, R., Strutt, H., and Strutt, D. (2003). Strabismus is asymmetrically localised and binds to Prickle and Dishevelled during Drosophila planar polarity patterning. Development 130, 3007-3014. 
Casal, J., Lawrence, P.A., and Struhl, G. (2006). Two separate molecular systems, Dachsous/Fat and Starry night/Frizzled, act independently to confer planar cell polarity. Development 133, 4561-4572.

Chae, J., Kim, M.J., Goo, J.H., Collier, S., Gubb, D., Charlton, J., Adler, P.N., and Park, W.J. (1999). The Drosophila tissue polarity gene starry night encodes a member of the protocadherin family. Development 126, 5421-5429.

Chen, W.S., Antic, D., Matis, M., Logan, C.Y., Povelones, M., Anderson, G.A., Nusse, R., and Axelrod, J.D. (2008). Asymmetric homotypic interactions of the atypical cadherin flamingo mediate intercellular polarity signaling. Cell 133, 1093-1105.

Cho, K.O., Hunt, C.A., and Kennedy, M.B. (1992). The rat brain postsynaptic density fraction contains a homolog of the Drosophila discs-large tumor suppressor protein. Neuron 9, 929-942.

Collier, S., and Gubb, D. (1997). Drosophila tissue polarity requires the cell-autonomous activity of the fuzzy gene, which encodes a novel transmembrane protein. Development 124 , 4029-4037.

Collier, S., Lee, H., Burgess, R., and Adler, P. (2005). The WD40 repeat protein fritz links cytoskeletal planar polarity to frizzled subcellular localization in the Drosophila epidermis. Genetics 169, 2035-2045. 
Das, G., Jenny, A., Klein, T.J., Eaton, S., and Mlodzik, M. (2004). Diego interacts with Prickle and Strabismus/Van Gogh to localize planar cell polarity complexes. Development 131, 44674476.

Das, G., Reynolds-Kenneally, J., and Mlodzik, M. (2002). The atypical cadherin Flamingo links Frizzled and Notch signaling in planar polarity establishment in the Drosophila eye. Dev Cell 2, 655-666.

Devenport, D., and Fuchs, E. (2008). Planar polarization in embryonic epidermis orchestrates global asymmetric morphogenesis of hair follicles. Nat Cell Biol 10, 1257-1268.

Etheridge, S.L., Ray, S., Li, S., Hamblet, N.S., Lijam, N., Tsang, M., Greer, J., Kardos, N., Wang, J., Sussman, D.J., et al. (2008). Murine dishevelled 3 functions in redundant pathways with dishevelled 1 and 2 in normal cardiac outflow tract, cochlea, and neural tube development. PLoS Genet 4, e1000259.

Feiguin, F., Hannus, M., Mlodzik, M., and Eaton, S. (2001). The ankyrin repeat protein Diego mediates Frizzled-dependent planar polarization. Dev Cell 1, 93-101.

Gho, M., and Schweisguth, F. (1998). Frizzled signalling controls orientation of asymmetric sense organ precursor cell divisions in Drosophila. Nature 393, 178-181. 
Gratz, S.J., Cummings, A.M., Nguyen, J.N., Hamm, D.C., Donohue, L.K., Harrison, M.M., Wildonger, J., and O'Connor-Giles, K.M. (2013a). Genome engineering of Drosophila with the CRISPR RNA-guided Cas9 nuclease. Genetics 194, 1029-1035.

Gratz, S.J., Wildonger, J., Harrison, M.M., and O'Connor-Giles, K.M. (2013b). CRISPR/Cas9mediated genome engineering and the promise of designer flies on demand. Fly (Austin) 7, 249255.

Gubb, D., and Garcia-Bellido, A. (1982). A genetic analysis of the determination of cuticular polarity during development in Drosophila melanogaster. J Embryol Exp Morphol 68, 37-57.

Hamblet, N.S., Lijam, N., Ruiz-Lozano, P., Wang, J., Yang, Y., Luo, Z., Mei, L., Chien, K.R., Sussman, D.J., and Wynshaw-Boris, A. (2002). Dishevelled 2 is essential for cardiac outflow tract development, somite segmentation and neural tube closure. Development $129,5827-5838$.

Jenny, A., Darken, R.S., Wilson, P.A., and Mlodzik, M. (2003). Prickle and Strabismus form a functional complex to generate a correct axis during planar cell polarity signaling. EMBO J 22, 4409-4420.

Jenny, A., Reynolds-Kenneally, J., Das, G., Burnett, M., and Mlodzik, M. (2005). Diego and Prickle regulate Frizzled planar cell polarity signalling by competing for Dishevelled binding. Nat Cell Biol 7, 691-697. 
Kim, E., and Sheng, M. (2004). PDZ domain proteins of synapses. Nat Rev Neurosci 5, 771781.

Klingensmith, J., Nusse, R., and Perrimon, N. (1994). The Drosophila segment polarity gene dishevelled encodes a novel protein required for response to the wingless signal. Genes Dev 8 , 118-130.

Lawrence, P.A., Casal, J., and Struhl, G. (2004). Cell interactions and planar polarity in the abdominal epidermis of Drosophila. Development 131, 4651-4664.

Lecker, S.H., Goldberg, A.L., and Mitch, W.E. (2006). Protein degradation by the ubiquitinproteasome pathway in normal and disease states. J Am Soc Nephrol 17, 1807-1819.

Lee, H., and Adler, P.N. (2002). The function of the frizzled pathway in the Drosophila wing is dependent on inturned and fuzzy. Genetics 160, 1535-1547.

Li, D., and Roberts, R. (2001). WD-repeat proteins: structure characteristics, biological function, and their involvement in human diseases. Cell Mol Life Sci 58, 2085-2097.

Lu, B., Usui, T., Uemura, T., Jan, L., and Jan, Y.N. (1999). Flamingo controls the planar polarity of sensory bristles and asymmetric division of sensory organ precursors in Drosophila. Curr Biol 9, 1247-1250. 
Lu, Q., Yan, J., and Adler, P.N. (2010). The Drosophila planar polarity proteins inturned and multiple wing hairs interact physically and function together. Genetics $185,549-558$.

Nagase, T., Ishikawa, K., Kikuno, R., Hirosawa, M., Nomura, N., and Ohara, O. (1999). Prediction of the coding sequences of unidentified human genes. XV. The complete sequences of 100 new cDNA clones from brain which code for large proteins in vitro. DNA Res 6, 337345.

Narimatsu, M., Bose, R., Pye, M., Zhang, L., Miller, B., Ching, P., Sakuma, R., Luga, V., Roncari, L., Attisano, L., et al. (2009). Regulation of planar cell polarity by Smurf ubiquitin ligases. Cell 137, 295-307.

Neer, E.J., Schmidt, C.J., Nambudripad, R., and Smith, T.F. (1994). The ancient regulatoryprotein family of WD-repeat proteins. Nature 371, 297-300.

Ran, F.A., Hsu, P.D., Wright, J., Agarwala, V., Scott, D.A., and Zhang, F. (2013). Genome engineering using the CRISPR-Cas9 system. Nat Protoc 8, 2281-2308.

Rodriguez-Gil, D.J., Hu, W., and Greer, C.A. (2013). Dishevelled proteins are associated with olfactory sensory neuron presynaptic terminals. PLoS One 8, e56561.

Semenov, M.V., and Snyder, M. (1997). Human dishevelled genes constitute a DHR-containing multigene family. Genomics 42, 302-310. 
Shimada, Y., Usui, T., Yanagawa, S., Takeichi, M., and Uemura, T. (2001). Asymmetric colocalization of Flamingo, a seven-pass transmembrane cadherin, and Dishevelled in planar cell polarization. Curr Biol 11, 859-863.

Singh, A., Chan, J., Chern, J.J., and Choi, K.W. (2005). Genetic interaction of Lobe with its modifiers in dorsoventral patterning and growth of the Drosophila eye. Genetics 171, 169-183.

Smith, T.F., Gaitatzes, C., Saxena, K., and Neer, E.J. (1999). The WD repeat: a common architecture for diverse functions. Trends Biochem Sci 24, 181-185.

Stirnimann, C.U., Petsalaki, E., Russell, R.B., and Muller, C.W. (2010). WD40 proteins propel cellular networks. Trends Biochem Sci 35, 565-574.

Strutt, D., and Warrington, S.J. (2008). Planar polarity genes in the Drosophila wing regulate the localisation of the FH3-domain protein Multiple Wing Hairs to control the site of hair production. Development 135, 3103-3111.

Strutt, D.I. (2001). Asymmetric localization of frizzled and the establishment of cell polarity in the Drosophila wing. Mol Cell 7, 367-375.

Sussman, D.J., Klingensmith, J., Salinas, P., Adams, P.S., Nusse, R., and Perrimon, N. (1994). Isolation and characterization of a mouse homolog of the Drosophila segment polarity gene dishevelled. Dev Biol 166, 73-86. 
Taylor, J., Abramova, N., Charlton, J., and Adler, P.N. (1998). Van Gogh: a new Drosophila tissue polarity gene. Genetics 150, 199-210.

Te Velthuis, A.J., Isogai, T., Gerrits, L., and Bagowski, C.P. (2007). Insights into the molecular evolution of the PDZ/LIM family and identification of a novel conserved protein motif. PLoS One 2, e189.

Tomlinson, A., and Struhl, G. (1999). Decoding vectorial information from a gradient: sequential roles of the receptors Frizzled and Notch in establishing planar polarity in the Drosophila eye. Development 126, 5725-5738.

Tree, D.R., Shulman, J.M., Rousset, R., Scott, M.P., Gubb, D., and Axelrod, J.D. (2002). Prickle mediates feedback amplification to generate asymmetric planar cell polarity signaling. Cell 109, 371-381.

Turner, C.M., and Adler, P.N. (1998). Distinct roles for the actin and microtubule cytoskeletons in the morphogenesis of epidermal hairs during wing development in Drosophila. Mech Dev 70, $181-192$.

Usui, T., Shima, Y., Shimada, Y., Hirano, S., Burgess, R.W., Schwarz, T.L., Takeichi, M., and Uemura, T. (1999). Flamingo, a seven-pass transmembrane cadherin, regulates planar cell polarity under the control of Frizzled. Cell 98, 585-595. 
Wang, Y., Hu, X.J., Zou, X.D., Wu, X.H., Ye, Z.Q., and Wu, Y.D. (2015). WDSPdb: a database for WD40-repeat proteins. Nucleic Acids Res 43, D339-344.

Wang, Y., Yan, J., Lee, H., Lu, Q., and Adler, P.N. (2014). The proteins encoded by the Drosophila Planar Polarity Effector genes inturned, fuzzy and fritz interact physically and can re-pattern the accumulation of "upstream" Planar Cell Polarity proteins. Dev Biol 394, 156-169.

Wolff, T., and Rubin, G.M. (1998). Strabismus, a novel gene that regulates tissue polarity and cell fate decisions in Drosophila. Development 125, 1149-1159.

Wong, H.C., Bourdelas, A., Krauss, A., Lee, H.J., Shao, Y., Wu, D., Mlodzik, M., Shi, D.L., and Zheng, J. (2003). Direct binding of the PDZ domain of Dishevelled to a conserved internal sequence in the C-terminal region of Frizzled. Mol Cell 12, 1251-1260.

Wong, L.L., and Adler, P.N. (1993). Tissue polarity genes of Drosophila regulate the subcellular location for prehair initiation in pupal wing cells. J Cell Biol 123, 209-221.

Yan, C., Wu, F., Jernigan, R.L., Dobbs, D., and Honavar, V. (2008a). Characterization of protein-protein interfaces. Protein J 27, 59-70.

Yan, J., Huen, D., Morely, T., Johnson, G., Gubb, D., Roote, J., and Adler, P.N. (2008b). The multiple-wing-hairs gene encodes a novel GBD-FH3 domain-containing protein that functions both prior to and after wing hair initiation. Genetics 180, 219-228. 
Yang, C.H., Axelrod, J.D., and Simon, M.A. (2002). Regulation of Frizzled by fat-like cadherins during planar polarity signaling in the Drosophila compound eye. Cell 108, 675-688.

Zeidler, M.P., Perrimon, N., and Strutt, D.I. (1999). The four-jointed gene is required in the Drosophila eye for ommatidial polarity specification. Curr Biol 9, 1363-1372.

Zheng, L., Zhang, J., and Carthew, R.W. (1995). frizzled regulates mirror-symmetric pattern formation in the Drosophila eye. Development 121, 3045-3055.

Zilber, Y., Babayeva, S., Seo, J.H., Liu, J.J., Mootin, S., and Torban, E. (2013). The PCP effector Fuzzy controls cilial assembly and signaling by recruiting Rab8 and Dishevelled to the primary cilium. Mol Biol Cell 24, 555-565. 


\title{
Chapter 4
}

The dynamics of the planar cell polarity effector protein Fritz in various tissues of Drosophila

\author{
Ying Wang
}




\begin{abstract}
The planar cell polarity effector genes inturned, fuzzy and fritz are downstream components in the frizzled/starry night signaling pathway, and their proper function is instructed and regulated by upstream PCP core genes such as frizzled, disheveled and van gogh. Previous research showed proteins encoded by PPE genes all asymmetrically accumulate on the proximal edge of wing cells and function in a protein complex by direct physical interactions. However, most of the previous studies about planar cell polarity genes used fixed tissues, which did not allow the dynamic properties of the PPE proteins and their asymmetric recruitment to be studied. We previously generated transgenes where myc-frtz-GFP was placed behind the ubiquitin promoter. The protein encoded by this gene (ubi-myc-frtz-gfp) provides complete rescue activity and accumulates on the proximal edge of wing cells. However, this transgene was inserted using standard P element germ line transformation, which results in a relatively random insertion in the genome. This inherently leads to varying expression levels between insertions. In order to study the dynamic recruitment of the PPE proteins in live cells, we made fusion PPE genes (Venus-in and frtz-mCherry) and used a variety of phi-C31 landing sites for insertion. Unfortunately, only one landing site (attpl) resulted in a level of expression where we could detect the zigzag accumulation pattern of PPE proteins. Even with this site the expression level was less than ideal for time lapse in vivo imaging. We next turned to using CRISPR/HDR to modify the frtz gene in the Drosophila genome by adding a green fluorescent protein mNeonGreen on its carboxyl terminus. This $f r t z^{m N e o n G r e e n}$ gene provides complete rescue activity and it works quite well for in vivo imaging. Our data shows Frtz is very dynamic in the cells and preferentially distributed to discrete membrane subdomains ("puncta"). We found Frtz accumulated in a zig-zag pattern in wing and thorax cells, and also accumulated asymmetrically
\end{abstract}


in pupal abdominal and arista cells. Fluorescence recovery after photo-bleaching study shows the recovery rate of Frtz protein concentrated at the edge of cells is similar in various tissues. 


\section{Introduction}

The coordination of cellular polarization is an important feature of animal development, and it is critical for patterning, maintenance and homeostasis of individual organs, tissues and the organism as a whole. Dysregulation of planar cell polarity (PCP) has been connected to various human diseases, including but not limited to neural tube defects, polycystic kidney disease, congenital heart disease, etc (Curtin et al., 2003; Fischer et al., 2006; Hamblet et al., 2002; Henderson et al., 2001; Henderson et al., 2006). Genetic studies revealed a number of genes responsible for regulating PCP using Drosophila wing model system, and the genes comprise the frizzled $(f z)$ /starry night (stan) signaling pathway (Adler et al., 1998; Chae et al., 1999; Collier and Gubb, 1997; Collier et al., 2005; Feiguin et al., 2001; Gubb and GarciaBellido, 1982; Klingensmith et al., 1994; Strutt and Warrington, 2008; Taylor et al., 1998; Wong and Adler, 1993; Yang et al., 2002; Zeidler et al., 1999). Based on their function and epistasis, these genes were placed into three phenotypic and epistasis groups. Planar cell polarity effectors (PPE) genes inturned (in), fuzzy (fy) and fritz (frtz) are downstream components in the $f z /$ stan signaling pathway (Collier et al., 2005; Wang et al., 2014; Wong and Adler, 1993). Our previous research showed in, fy and frtz form a protein complex and function together in the wing cells (Wang et al., 2014). PPE proteins are recruited to the proximal side of wing cell by the upstream PCP core proteins, such as Disheveled (Dsh) (Chapter 3). However, we don't know how the recruitment happens. It is also not known how the PPE proteins are recruited to the proximal side of wing cells - individually or in a protein complex.

In order to understand the dynamic properties of PCP and PPE proteins, time-lapse in vivo imaging is required. The most commonly used technique for this uses a transgenically encoded protein fused to a fluorescent protein. In this chapter, I describe the methods for 
generating the PPE transgenic stocks with different fluorescent tags and their potential for timelapse imaging. We made fusion PPE proteins Venus-In and Frtz-mCherry, and the attpl landing site insertion using phi-C31 integrase results in a typical zigzag accumulation pattern of In and Frtz, respectively. Using these stocks will allow me to image PPE proteins In and Frtz together to study how the proteins or protein complex are recruited asymmetrically, although the expression level was less than ideal for time lapse in vivo imaging.

Over the past decade, efficient genome editing has been developed for a wide range of experimental systems ranging from plants to animals. The commonly used genome editing techniques include but are not limited to Zinc finger nucleases (ZFNs), (Durai et al., 2005; Kandavelou and Chandrasegaran, 2009) transcription-activator like effector nucleases (TALEN) (Boch, 2011; Boch et al., 2009) and meganucleases (Epinat et al., 2003). Recently, researchers discovered a much cheaper and easier gene editing tool, CRISPR/Cas9, to cut or label a gene of interest. RNA-guided Cas9 nuclease from the microbial clustered regularly interspaced short palindromic repeats (CRISPR) adaptive immune system can be used to facilitate efficient genome engineering in eukaryotic cells by simply specifying a 20-nt targeting sequence within its guide RNA (gRNA) (Cong et al., 2013; Gratz et al., 2013a; Gratz et al., 2013b; Ran et al., 2013).

In this chapter, I also describe the use of this method for modifying the frtz gene in the genome. I added a green fluorescent protein mNeonGreen onto carboxyl terminus of frtz gene and this transgene provided complete rescue of a frtz loss-of-function (lof) mutant. In vivo imaging of the Frtz ${ }^{\text {meonGreen }}$ showed that Frtz accumulates in a zigzag pattern in wing cells and thorax cells. We also observed the asymmetric accumulation of the Frtz protein in abdominal and arista cells which haven't been reported before this thesis. Using time-lapse in vivo 
imaging, we found the Frtz protein is very dynamic within all cells we examined (wing, thorax, abdominal and arista cells) and preferentially distributed to discrete membrane subdomains. Previous research in Strutt lab shows the two PCP core proteins frizzled $(f z)$ and starry night (stan) have low recovery after photo-bleaching (about $20 \%$ recovery for $f z$ and $45 \%$ for stan), indicating that overall $f z$ and stan turnover is low (Strutt et al., 2011). Fluorescence recovery after photo-bleaching (FRAP) studies using various cell types shows Frtz protein recovers rapidly after photo-bleaching and their recovery rate are similar. However, there is no a complete recovery of the fluorescence (about $80 \%$ recovery), suggesting there are both stable and less stable Frtz protein pools within the cells. 


\section{Methods and Materials}

\section{Antibodies}

Anti-Myc antibody was obtained from Cell Signaling Technology. Anti-GFP antibody was obtained from Molecular Probes. Alexa 488- and Alexa 568-conjugated secondary antibodies were purchased from Molecular Probes.

\section{Immunostaining}

A standard paraformaldehyde fixation and staining procedure was used (Adler et al., 2004). Briefly, fly pupae were fixed in $4 \%$ paraformaldehyde, $\mathrm{PBS}$ for $2-3 \mathrm{hr}$ at $4{ }^{\circ} \mathrm{C}$. After fixation, wings were dissected, rinsed, blocked and then stained with primary antibodies in PBS, $0.3 \%$ Triton X-100 and 10\% goat-serum overnight at $4{ }^{\circ} \mathrm{C}$. Secondary antibodies were applied for 2-3 hr at room temperature.

\section{Frtz protein live imaging}

$$
\text { Frtz }^{\text {mNeonGreen }}, \text { ubi-myc-frtz-gfp, ubi-myc-venus-in and ubi-myc-frtz-mcherry white pupae }
$$
were collected then aged at $25^{\circ} \mathrm{C}$ for either $30 \mathrm{hr}$ for imaging wing, thorax and arista cells or for about $42 \mathrm{hr}$ for imaging abdominal cells. Aged pupae were placed on double-side tape attached on microscope slides. The pupae cases were peeled back over the region to be imaged. Two silicon rubbers were used to build a chamber and one drop of halocarbon oil was applied to the cover slip. Zeiss 780 microscope $63 x$ or $40 \mathrm{x}$ oil immersion lens were used.

\section{Plasmid constructs}

\section{pWUM6- frtz-mCherry construct}


pWUM6 vector was digested with EcoRI and $\mathrm{XbaI}$ restriction enzymes and the fragments were amplified using the following primers and assembled into the digested vector using Gibson Assembly.

Primers for amplifying frtz fragment

frtz5': CGGCGTAGATCTGGTACCTATGCTGCTCAGCGAGACCCATTT

frtz3': CTCCTCGCCCTTGCTCACCATGCGATCGCTGACCACGCCGAAGTGGACCA

Primers for amplifying $m$ Cherry fragment

mcherry5':

TGGTCCACTTCGGCGTGGTCAGCGATCGCATGGTGAGCAAGGGCGAGGAG

mcherry3': CGGCATGGACGAGCTGTACAAGTAACTAGGCCGGCCTTCAAGGCCT

\section{pWUM6 -Venus-in construct}

$p W U M 6$ vector was digested with corresponding restriction enzymes and the fragments were amplified using the following primers and ligated into the digested vector.

Primers for amplifying Venus fragment

Venus 5': CTAGTCTAGAATGGTGAGCAAGGGCGAGGAG (XbaI)

Venus3' : ATAAGAATGCGGCCGCCGTGGACCGGTGCTTGTACAGCTC (NotI)

Primers for amplifying In fragment 
In 5: ATAAGAATGCGGCCGCATGCGCAAATCGCCGGCCAG (NotI)

In 3: CGACGCGTTTATCCCATTGAGAAGAAGGACA (MluI)

$f r t z^{\text {mNeonGreen }}$ Crispr gRNA plasmid construction :

gRNA primers:

PCFD4 FRTZ 5:

TATATAGGAAAGATATCCGGGTGAACTTCGCCGCCCATCCGACGACGCGGTTTTAG AGCTAGAAATAGCAAG

PCFD4 FRTZ 3:

ATTTTAACTTGCTATTTCTAGCTCTAAAACCACAACGGTTAAGCGCTGCCGACGTTA AATTGAAAATAGGTC

The above primers amplified a 600bp fragment by PCR which includes both 5gRNA and 3gRNA. The fragment was inserted into BbsI digested pCFD4-U6:1_U6:3 tandem gRNAs vector (Addgene plasmid \# 49411) and ligated by T4 DNA ligase.

frt $^{\text {mNeonGreen }}$ Crispr HDR template construction

5' flanking region primers

5+7th exon 5': CCGGAATTCTCGGCGGTGACCAGCGACGA (EcoRI)

5+7th exon 3': ATAAGAATGCGGCCGCGACCACGCCGAAGTGGACCA (NotI)

mNeonGreen primers:

mNeonGreen 5': ATAAGAATGCGGCCGCATGGTGAGCAAGGGCGAGGA (NotI) 
mNeonGreen 3': CTACATATGTTACTTGTACAGCTCGTCCA (NdeI)

3' flanking region primers

3 arm 5': GGAAGATCTAGGCGTACACCACACAACGG (BglII)

3 arm 3': CCGCTCGAGCGCCGGGCCAAGTAGCAAGG (XhoI)

Both 5' and 3' flanking regions were amplified by PCR using genomic DNA.

mNeonGreen clone was purchased from Allele Biotech and amplified by PCR. The fragments were digested with corresponding restriction enzymes and ligated into the same restriction enzymes digested $p H D$-DsRed (Addgene ) vector. The 3 nucleotides PAM sequence following the gRNA on the HDR template were mutated so the Cas9 nuclease would not recut edited DNA.

Transgenic DNA injection was performed at Rainbow Transgenic Flies, Inc . Both gRNA plasmid and HDR template were injected into nos-cas9 attp2 (21104) stock. The Go progeny carrying the inserts were selected by Ds-Red fluorescence and confirmed by genomic DNA PCR.

\section{Quick Preparation of Genomic DNA from Drosophila}

Buffer A (store at room temperature)

$100 \mathrm{mM}$ Tris-Cl (pH 7.5)

100 mM EDTA

$100 \mathrm{mM} \mathrm{NaCl}$

$0.5 \%$ SDS 
Buffer B (store at $4^{\circ} \mathrm{C}$ )

$200 \mathrm{~mL}$ potassium acetate $(5 \mathrm{M})$

$500 \mathrm{~mL}$ lithium chloride $(6 \mathrm{M})$

Mix together and store at $4^{\circ} \mathrm{C}$.

1. Collect 30 anesthetized flies in a $1.5-\mathrm{mL}$ microcentrifuge tube placed on ice. Flies can be stored at $-80^{\circ} \mathrm{C}$ indefinitely or DNA can be prepared immediately without freezing the flies.

2. Grind flies in $200 \mu \mathrm{L}$ of Buffer A with a disposable tissue grinder. Add an additional $200 \mu \mathrm{L}$ of Buffer A (to a total volume of $400 \mu \mathrm{L})$ and continue grinding until only cuticles remain $(\sim 1-2$ min, grinding by hand).

3. Incubate samples at $65^{\circ} \mathrm{C}$ for $30 \mathrm{~min}$.

4. Add $800 \mu \mathrm{L}$ of Buffer B to each sample, mix well by inverting the tube multiple times, and incubate on ice for at least 10 min and up to a few hours.

5. Centrifuge in a microcentrifuge at $12,000 \mathrm{rpm}$ for $15 \mathrm{~min}$ at room temperature.

6. Transfer $1 \mathrm{~mL}$ of the supernatant into a new microcentrifuge tube (some of the precipitate will not pellet, but instead float on top of the supernatant; be extremely careful to avoid transferring any floating precipitate). Discard the pellet. If necessary, repeat the centrifugation step to remove any contaminating precipitate.

7. Add $600 \mu \mathrm{L}$ of isopropanol to each sample, and mix well by inverting the tube several times.

8. Centrifuge at $12,000 \mathrm{rpm}$ for $15 \mathrm{~min}$ at room temperature. 
9. Discard the supernatant. Wash the pellet with $70 \%$ ethanol, air-dry, and resuspend in $150 \mu \mathrm{L}$ of TE buffer.

10. Store the DNA at $-20^{\circ} \mathrm{C}$. 


\section{Results}

\section{Asymmetric accumulation of Frtz protein}

The planar cell polarity effectors (PPE) in, fy and frtz share a very similar loss-offunction phenotype in which multiple hairs are formed within a substantial number of wing cells, and their polarity are changed. Figure 1A shows the frtz loss-of-function phenotype. Both wing hair polarity and numbers are abnormal. To determine the subcellular location of Frtz, the stock Ubi-myc-frtz-GFP was generated in our lab and it rescued frtz null mutant (figure 1B). The rescue test suggests the fusion protein is fully functional. The ubiquitin promoter used in the vector has been proven in the past to drive expression at a modest level that doesn't result in over-expression phenotype (Sekelsky et al., 1995). Using the stock Ubi-myc-frtz-GFP, Jie Yan observed that Frtz localized asymmetrically and showed a zigzag pattern on the apical surface of pupal wing cells (Figure 1C). In order to know if Frtz was localized at the proximal, distal or both sides of cell, we examined pupal wing cells expressing the fusion protein in a variegated pattern. Frtz was tagged with both Myc and GFP tag. GFP tag expressed homogeneously in the wing, but Myc tag expressed in a mosaic pattern due to some unknown reasons. From cells located at the boundaries, we found Frtz protein was only accumulated on the proximal side (Figure 1D).

\section{Live imaging of transgenic Frtz protein}

To understand the dynamic properties of Frtz in live cells, I used the Ubi-myc-frtz-GFP transgenic fly. The in vivo imaging shows that Frtz protein accumulates in a zig-zag pattern, as expected based on experiments with fixed cells (Figure 2A). In addition, Frtz protein is

preferentially distributed to discrete membrane subdomains ("puncta"), and the puncta are very dynamic at the subcellular level. Various types of movements were observed for the "puncta" 
through time-lapsed imaging. Figure 2 shows several examples of the movement pattern. For example, we observed fusion of two small puncta into a larger one (Figure 2B), or the split of a large puncta into two smaller ones (Figure 2D). We also observed the puncta going off or moving back to cell boundary (Figure 2C). Fluorescence recovery after photo-bleaching (FRAP) was done to measure the Frtz protein turnover rate (Figure 2E). FRAP data shows that bleached area in wing cells was almost fully recovered within 6 mins after photo-bleaching and the estimated recovery half time is $90 \mathrm{~s}(\mathrm{n}=8)$, suggesting membrane bound Frtz protein rapidly exchanges with soluble protein (Figure $2 \mathrm{~F}$ )

\section{Generation of transgenic flies with tagged PPE proteins}

Our previous research showed PPE proteins are recruited to proximal side of the wing cell and form a protein complex there (Wang et al., 2014). However, when and how the protein complex is formed is unknown. It may forms before or after the recruitment. In order to know more detailed information about the dynamic properties of the PPE proteins recruitment, the flies expressing transgenic PPE proteins with different color fluorescent tags were generated. The red fluorescent protein mCherry, cyan fluorescent protein mCerulean3 and yellow fluorescent protein Venus were tagged to the carboxyl terminus of Frtz, Fy and In, respectively. The transgenic PPE proteins expression was driven by ubiquitin promoter and the DNA were inserted to the same location on Drosophila genome using phiC31 integrase to minimize the variation of protein expression level at different genomic locations. Since ubi-myc-frtz-gfp was inserted into Drosophila genome randomly, various landing sites were tested to duplicate this expression. attpl, attp2 and vk00027 landing sites were tried and only attpl landing site insertion shows a typical zigzag accumulation pattern of Frtz and In proteins (Table 1). Transgenes inserted into other sites gave very low expression levels. We also noticed that 
putting the Venus tag on the amino terminus of In gave a higher level of protein accumulation compared to when it was linked to the carboxyl terminal end. This may due to the stabilization

of In protein by amino-tagged protein (Table 1). Fy-mCerulean3 plasmid was injected to both attp 1 and vk00027 landing sites and neither show a good zig-zag accumulation pattern. Previous attempts in our lab to detect tagged transgenic Fy in a zigzag pattern were also not successful, so further attempts to generate $f y$ transgenic lines was not continued. Figure 3 shows the immunostaining images of Venus-In and Frtz-mCherry. The time-lapse imaging data shows the accumulation of both proteins are dynamic and form "puncta" in the cell as well (data not shown here), but the expression level is weaker than $u b i-m y c-f r t z-g f p$.

\section{Modifying frtz in the genome using CRISPR/Cas9 genome editing technique}

The tagged PPE transgenes were less than ideal for several reasons including: 1) since the level of gene expression was not at the endogenous level there is always a chance that our results will be misleading due to expression level issues. 2) Our unpublished data suggests ubiquitin promoter drives a relative high protein expression level in some tissues in the abdomen, which makes it difficult to use transgenes incorporating this enhancer/promoter for studying the role of the PPE genes in PCP in the abdomen. 3) Past attempts to raise antibodies against the Frtz protein did not result in a reagent that could detect the endogenous protein. Thus, we were unable to determine the endogenous pattern of Frtz accumulation. In order to study the activity of the native PPE proteins, I took the advantage of the CRISPR/Cas9 nuclease genome editing technique to add a green fluorescent protein, mNeonGreen, to the carboxy terminus of the endogenous frtz gene. There are seven exons in frtz gene. Two gRNA constructs were designed to specifically cut the $7^{\text {th }}$ exon of frtz. The homology directed repair (HDR) template including a mNeonGreen sequence, a DS-Red sequence and two $1.5 \mathrm{~kb}$ homology arms 
flanking both end of the cut and $f r t z 7^{\text {th }}$ exon was designed to repair the gap introduced by the two gRNAs. The DS-Red fluorescence marker was used to screen the G0 progeny for the ones that carried the edited gene.

The G0 flies from the CRISPR injection were first crossed to $w ; C y O / G l a$ flies and the G1 progeny were screened for Ds-Red fluorescence. Genomic DNA from adult flies with DsRed fluorescence was extracted and PCR done to validate edited genes. 50\% of G0 progeny gave rise to G1 progeny that displayed Ds-Red fluorescence and $20 \%$ of those had the insertion of the mNeonGreen sequence at the correct genomic location. Figure 4A shows the frtz gene map in the genome and the two gRNAs targeting regions. The design of the HDR template and the replaced genomic sequence were also include in figure 4A. The newly generated $\mathrm{frtz}^{\text {mNeonGreen }}$ stock rescued the frtz loss-of-function mutant suggesting the fusion protein is fully functional. Figure 4B shows the wing hair phenotype from the rescued flies. Two regions on the wing were chosen to illustrate the rescue.

\section{Frtz protein asymmetrically accumulates in various tissues of Drosophila and very} dynamic in cells

Strutt and colleagues reported the asymmetric accumulation of Frtz in wing cells by immunostaining a Frtz transgenic protein (Strutt and Warrington, 2008) and we also obtained this result using $u b i-m y c-f r t z-g f p$ transgenic flies. To determine if the endogenous Frtz protein has the same accumulation pattern, $f r t z^{\text {mNeonGreen }}$ fly was used for live imaging and various tissues were examined. Not surprisingly, we observed the same asymmetric accumulation pattern for Frtz protein in both wing cells and thorax cells around 30hr apf (after puparium formation) (Figure 5A\&B). At later stages we also observed Frtz along the cell periphery, but the zigzag pattern was no longer distinct and the accumulation was relatively uniform. Around 
$43 \mathrm{hr}$ apf, we observed the asymmetric accumulation of Frtz protein in abdominal cells (Figure 5C). This had not been seen previously.

In order to know if there is a difference in the dynamic properties of the Frtz protein in different tissues, FRAP analysis was done using wing, thorax and abdominal cells. After bleaching the cells, the fluorescence substantially recovered in all three tissues within 60s (Fig $5 \& 6)$ and the estimated recovery half time is 70 s. However, there was no a complete recovery of the fluorescence. It suggests the cells consist of both stable and less stable Frtz protein pools. The stable protein has low recovery once bleached, but the less stable protein could recover rapidly and fully recovered.

Previous research showed the $f z /$ stan signaling pathway regulates the development of Drosophila arista. Mutations in the PPE genes in and fy resulted in production of multiple and split laterals (He and Adler, 2002). Using frtz ${ }^{\text {mNeonGreen }}$ fly, we successfully detect the asymmetric accumulation of Frtz in arista cells (Figure 5D) and further validate their conclusion that PPE proteins also function in Drosophila arista development. 


\section{Discussion}

\section{Accumulation of Frtz in other tissues}

In this chapter I described the asymmetric accumulation of Frtz protein in wing, thorax,

arista and abdominal cells. However, our $f r t^{-m N e o n G r e e n}$ fly can be used to study the accumulation of Frtz in other tissues and structures, such as the developing eye and Drosophila leg cells, etc. A substantial advantage to use the $\mathrm{frtz}^{\text {mNeonGreen }}$ carrying flies to study the Frtz protein function is that its expression is expected to be the same as the wild type gene.

\section{Uses of $\mathrm{frtz}^{\text {mNeonGreen }}$ for studying the PPE protein dynamics}

Previous research showed that the PPE proteins In and Frtz accumulated asymmetrically on the proximal edge of the wing cells and form a protein complex there. We also found the accumulation of one PPE protein can modulate the accumulation of the others (Wang et al., 2014). However, we don't know how the PPE proteins In and Frtz are recruited to the proximal edge and when the protein complex is formed. One hypothesis is that In and Frtz may form the protein complex before they are recruited to the proximal side. Alternatively, they could be recruited individually to the proximal side of the cell first and then form a protein complex there. In order to know which one is correct, modified in gene is required. By adding a red fluorescent tag to in coding region using CRISPR/Cas 9 genome editing tool and imaging both Frtz and In together, we will be able to visualize the dynamic property of PPE protein recruitment. The two gRNA constructs and HDR template for modifying in gene using CRISPR technique were generated and injected into Cas9 containing embryos. I will soon be screening 
for Ds-Red expression to identify candidate edited genes. This will give us an In protein tagged with mRuby 2 that is expressed by the endogenous regulatory machinery.

\section{Two pools of Frtz protein within the cells}

In this chapter, I described the FRAP analysis of the Frtz protein using both ubi-mycfrtz-gfp and $f r t z^{m N e o n G r e e n}$ flies. Using $u b i-m y c-f r t z-g f p$ fly, we observed almost full recovery of the Frtz protein after photo-bleaching. However, there was only about $80 \%$ of the Frtz protein

recovered in various tissues of $f r t z^{m N e o n G r e n}$ fly. Our hypothesis is that there are two pools of Frtz protein within the cells, stable and less stable Frtz. The Frtz protein is slightly over expressed in $u b i-m y c-f r t z-g f p$ transgenic fly and that leads to an increased pool of protein that is not localized and that helps with recovery. However, there might not be enough soluble Frtz ${ }^{\mathrm{mNeonGreen}}$ protein to provide full recovery after photo-bleaching.

\section{Comparison of PPE and PCP core proteins recovery after photo-bleaching}

Strutt and colleagues did FRAP analysis on the PCP core proteins Fz and Stan, and their data suggest Fz and Stan proteins accumulated near the adherens junctions are highly stable (Strutt et al., 2011). Only a small fraction of $\mathrm{Fz}$ and Stan proteins recovered after photobleaching. However, our FRAP analysis on Frtz protein shows its dynamic accumulation within different cell types. Out data shows about $80 \%$ of the fluorescence recovered after photobleaching. Although not fully recovered, cytoplasmic Frtz appears more dynamic than the transmembrane PCP core proteins Fz and Stan. There is about 20\% of the Frtz protein lost after photo-bleaching. Although there is a small chance that $5 \mathrm{~min}$ is insufficient for allowing the fully recovery of Frtz protein, it is most likely because this $20 \%$ of the Frtz protein is more stable than the remainder. After being bleached, the Frtz protein remains in its location on the 
proximal edge of wing cells and is not replaced by newly synthesized fluorescent Frtz. This stable Frtz pool represents a smaller fraction of the localized protein than is seen for the stable Fz and Stan proteins. 
Figure 1.Frtz accumulates at the proximal edge of wing cells. Panels A and B are the same region of adult wings showing the rescue activity of the $u b i-m y c-f r t z-G F P$ transgene. Panel A is frtz/frtz, and panel B is frtz/frtz; ubi-myc-frtz-GFP/+. Note that the frtz transgene rescues the $f r t z$ mutant phenotype. Panels C-D are pupal wings, containing the ubi-myc-frtz-GFP transgene, stained with an anti-GFP antibody (Green) or an anti-Myc antibody (Red). Frtz accumulates asymmetrically and shows a zigzag pattern in $\mathrm{C}$. The region of the wing shown in panel D shows variegated expression of the transgene, and this allowed us to determine that Frtz accumulates on the proximal side of pupal wing cells (see arrows). In panel D distal is to the right and proximal to the left. This figure was done by Jie Yan. 

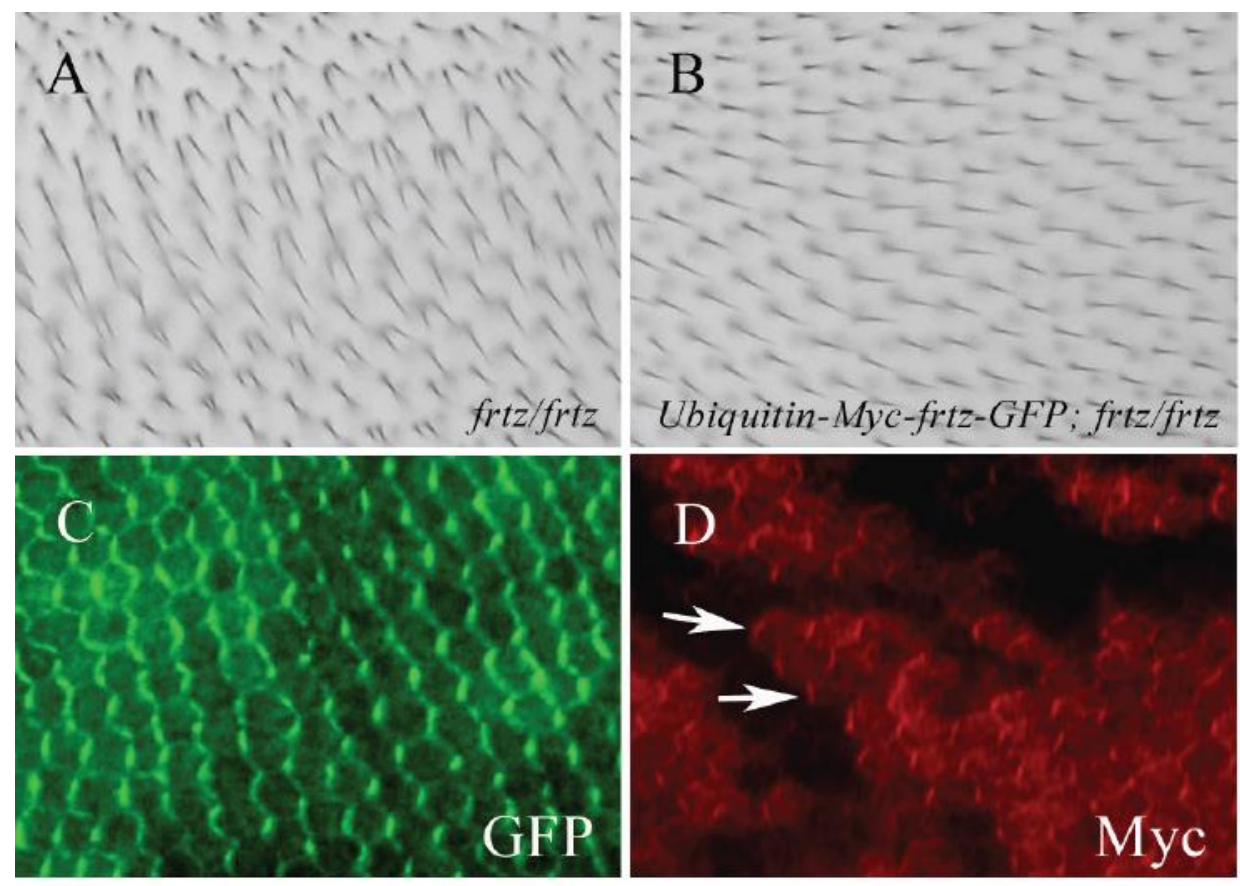
Figure2. Live cell imaging of $u b i-m y c-f r t z-g f p$. A shows asymmetric accumulation of Frtz in wing cells. B-D show examples of Frtz protein dynamic movements in wing cells using timelapse imaging. B Shows the fusion of two small protein particles into a larger one. C shows a protein particle moving off the cell boundary. D shows that a larger protein particle splitting into two smaller ones. E \& F show FRAP analysis of thorax cells. E shows an example of the protein recovery in a bleached area within 2 mins. The white rectangle area was bleached with $100 \%$ intensity $488 \mathrm{~nm}$ laser. The fluorescence was measured along the bleached cell boundary using a 5-pixel line. F shows the average recovery of Frtz protein at 8 regions within 6 mins. Data was normalized to auto-bleaching of Frtz protein in unbleached areas. White pupae were aged at $25^{\circ} \mathrm{C}$ for $30 \mathrm{hr}$. Wing and thorax cells were imaged using Zeiss $78063 \mathrm{x}$ oil lens. 


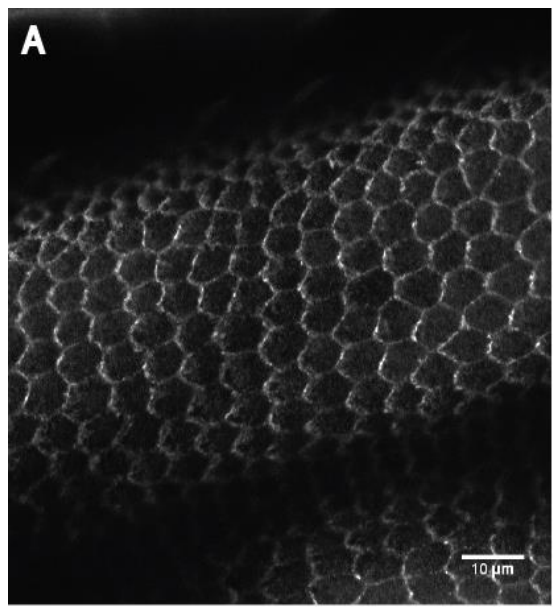

B. Fusion of two protein particles
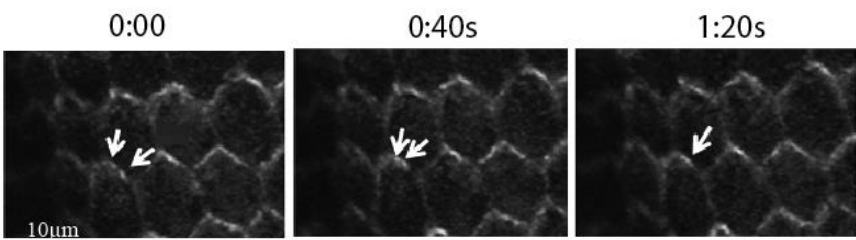

C. Particle moving off the cell boundary

$0: 00$

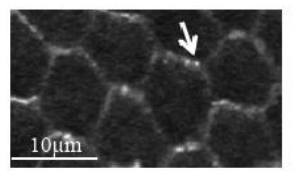

$0: 40$

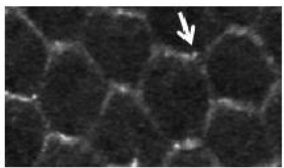

$1: 20$

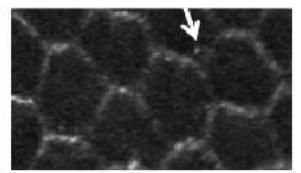

D. Protein particle split

0:00

$0: 40 \mathrm{~s}$

$1: 20 \mathrm{~s}$

2:00s
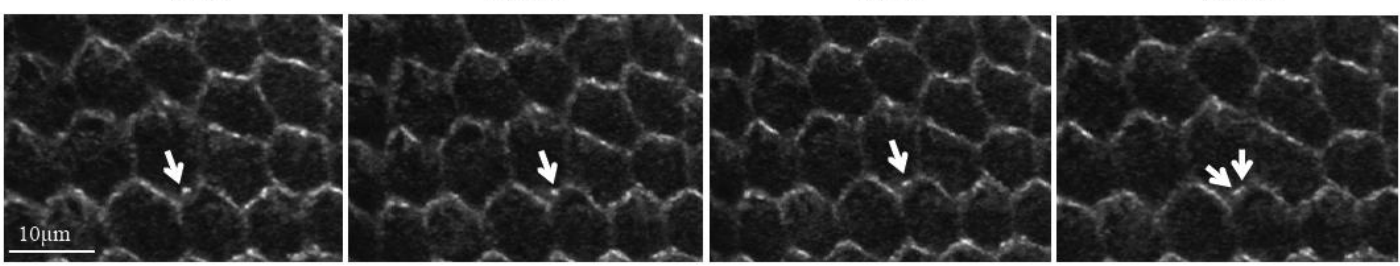

E. ubi-myc-frtz-gfp fluorescence recovery after photo-bleaching (FRAP)

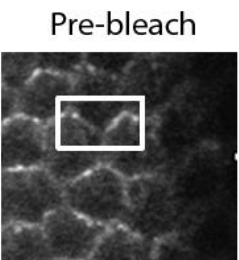

01:00

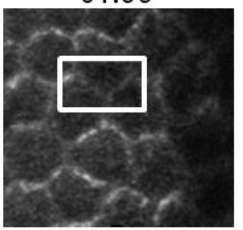

F

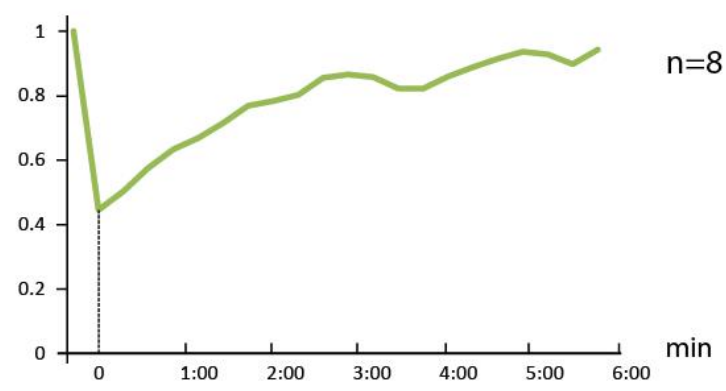

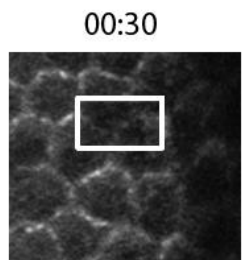

$01: 45$
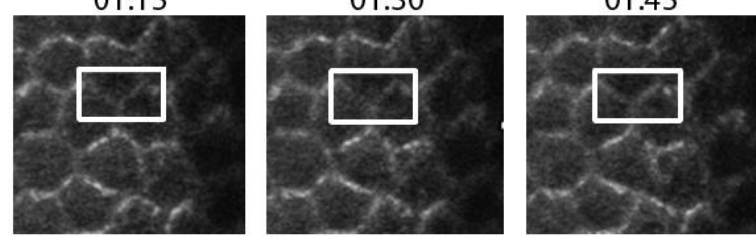

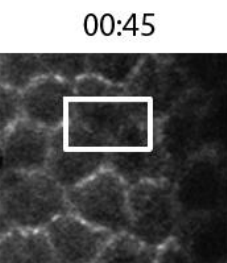

02:00

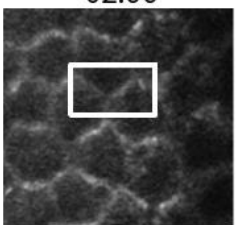


Figure 3. Live cell imaging of Venus-In (A) and Frtz-mCherry (B). White pupae of $p W U M 6$ venus-in and $p W U M 6$-frtz-mCherry were aged at $25^{\circ} \mathrm{C}$ for $30 \mathrm{hr}$ and thorax cells were imaged using Zeiss 780 63x oil lens. 

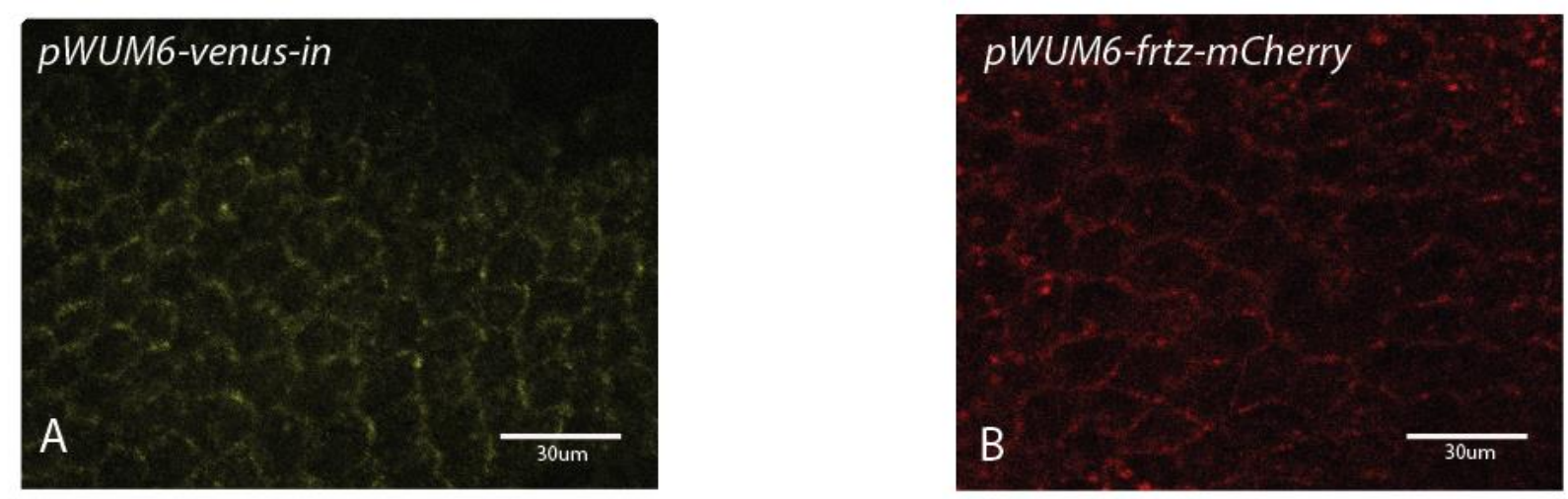
Figure 4. $f r t z^{-m N e o n G r e e n}$ CRISPR design and the rescue of the frtz lof mutant. A. (i) shows the frtz gene map in the genome and the two gRNAs targeting regions (marked by red X). (ii) shows the design of the HDR template. Two $1.5 \mathrm{~kb}$ homolog arms were amplified from frtz 7 th exon and its flanking regions on both ends. mNeongreen sequence was ligated after the frtz 7 th exon and kept in frame with Frtz protein. Ds-Red fluorescent sequence was inserted between two homolog arms which can be further removed by lox/cre recombination. The gene region between two dash lines in (i) is replace by sequence in (ii) after homolog directed repair. B. $f r t z^{\text {mNeonGreen }}$ fly rescued the frtz lof mutant. The $f r t z^{\text {mNeonGreen }}$ fly was crossed with frtz-/-. All progeny have a wild-type phenotype. 
A

(i)

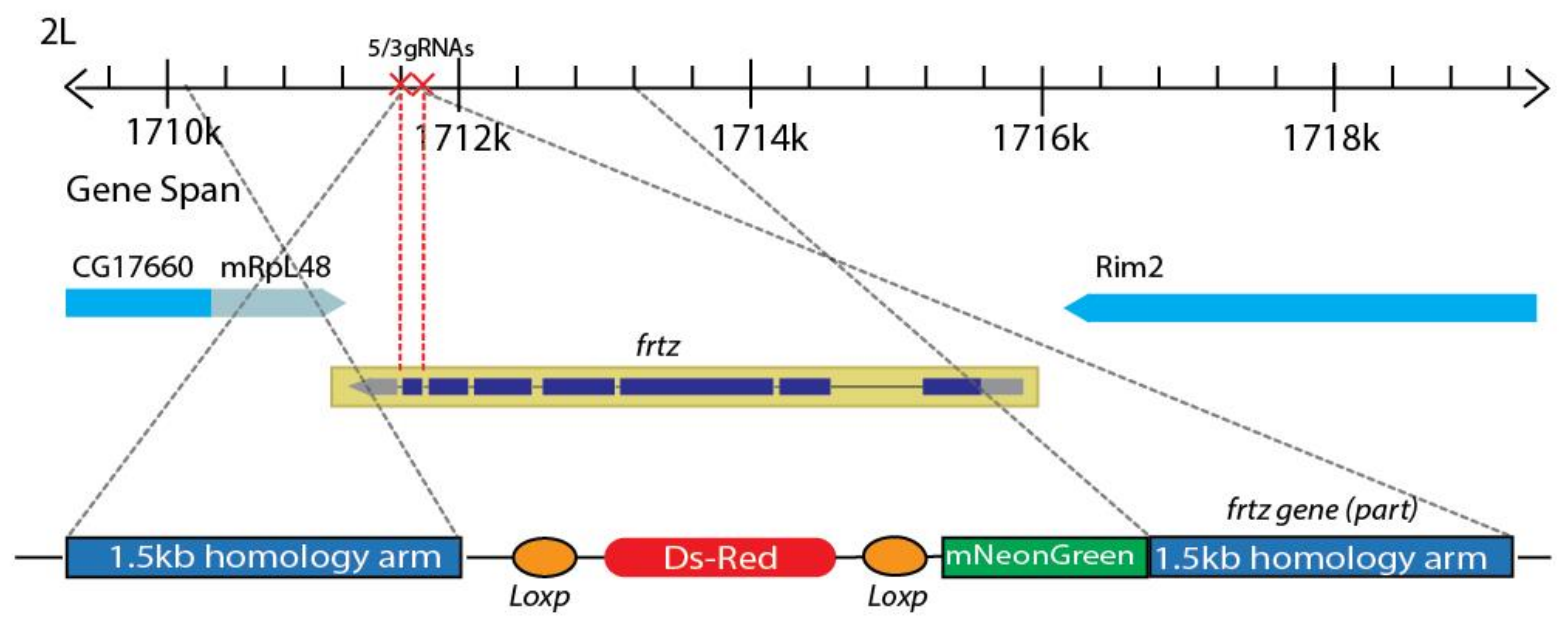

B

frtz-mNeonGreen/frtz'

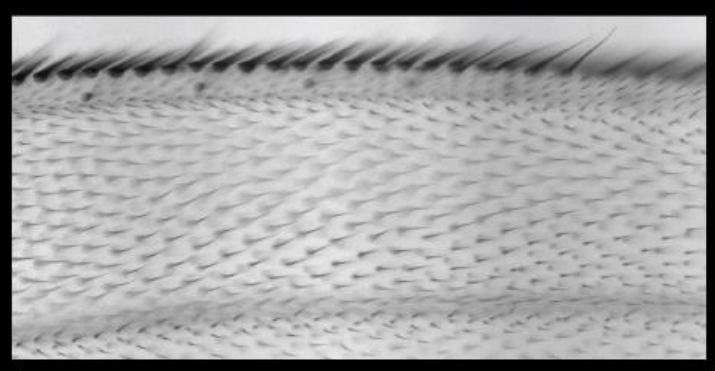

frtz $^{-} /$frtz $^{-}$
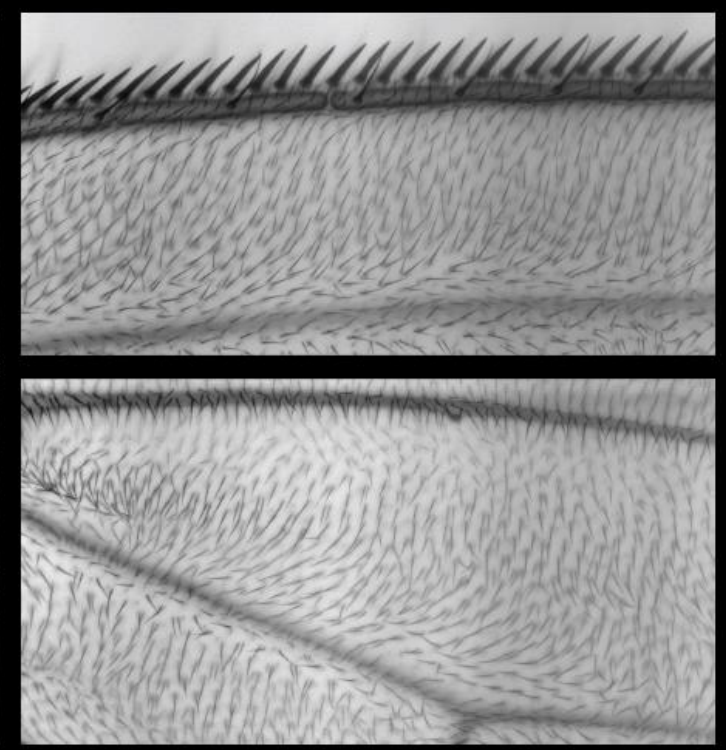
Figure 5. Asymmetric accumulation of Frtz in various tissues and FRAP analysis. A-D are the asymmetric accumulation of Frtz in wing cells (A), thorax cells (B), abdominal cells (C) and arista cells (D). E shows the comparison of Frtz protein recovery after photo-bleaching in wing cells, thorax cells and abdominal cells. 15 regions were bleached in each tissue and the average number was graphed. The fluorescence was measured along the bleached cell boundary using a 5-pixel line. Figure F-J show the Frtz protein recovery in abdominal cells within 2 min. F shows the pre-bleached cells. Arrows point to the bleached area. White pupae were aged at $25^{\circ} \mathrm{C}$ for 30hr for wing, thorax and arista cells, $42 \mathrm{hr}$ for abdominal cells. Wing, thorax and abdominal cells were imaged using Zeiss 780 63x oil lens. Arista cells were imaged using 40x oil lens. Cells were photo-bleached using $100 \%$ intensity of 488nm laser. 
Wing

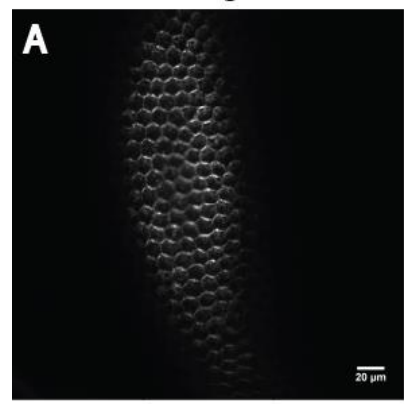

E

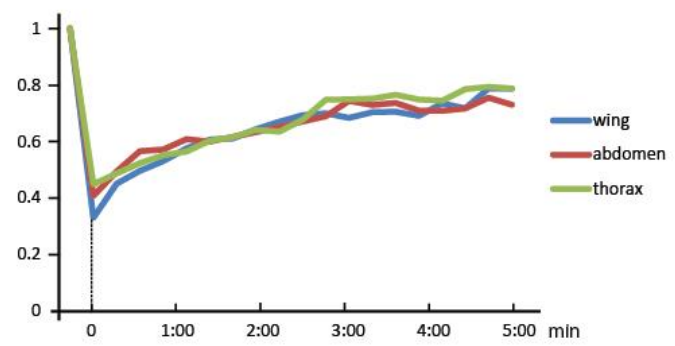

pre-bleach

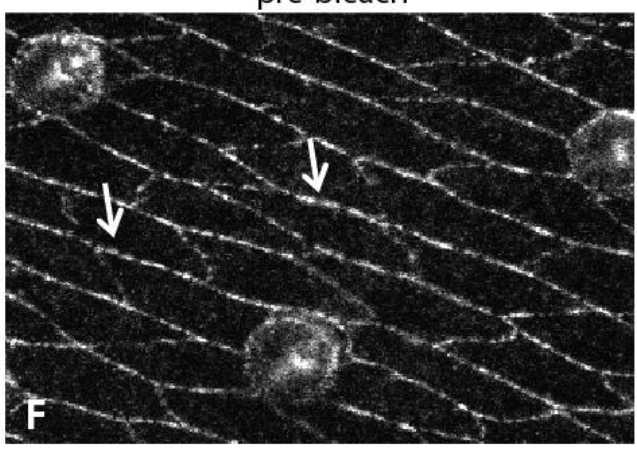

00:30 min

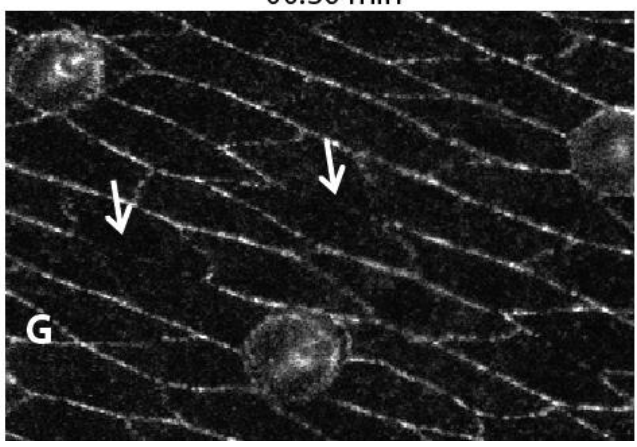

Abdomen

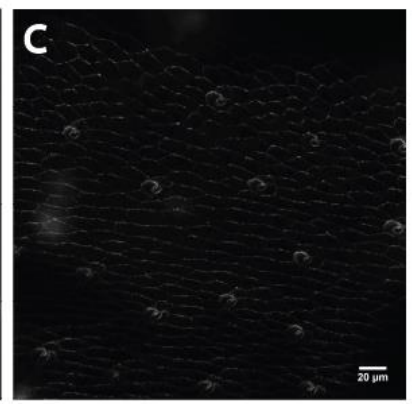

1:00 min

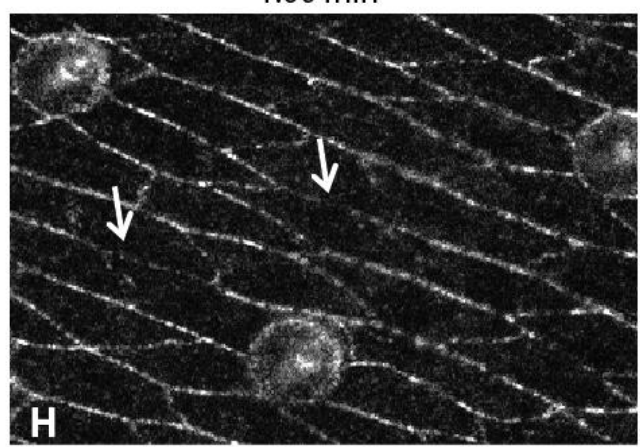

1:30 min

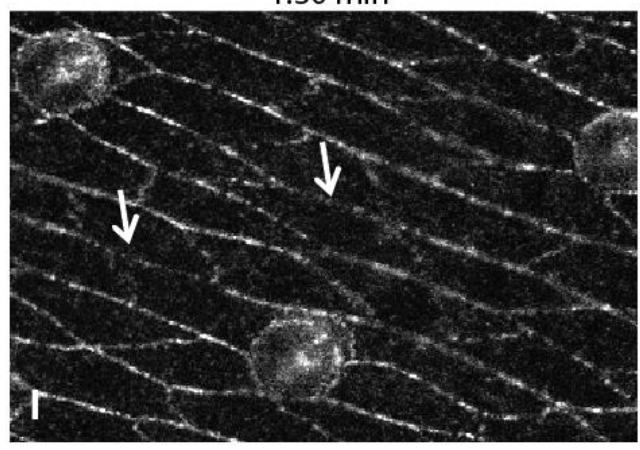

2:00 min

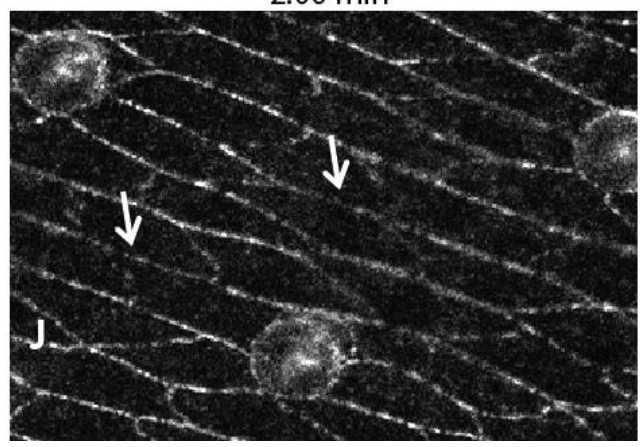


Figure 6. A-E show Frtz protein recovery in wing cells within 1:30 min. Figure F-J show Frtz protein recovery in thorax cells within 1:30 min. A\&F shows the pre-bleached cells. Arrows point to the bleached area. White pupae were aged at $25^{\circ} \mathrm{C}$ for $30 \mathrm{hr}$ for wing and thorax cells. Cells were imaged using Zeiss 780 63x oil lens. Cells were photo-bleached using 100\% intensity of $488 \mathrm{~nm}$ laser. 
wing cell

pre-bleach
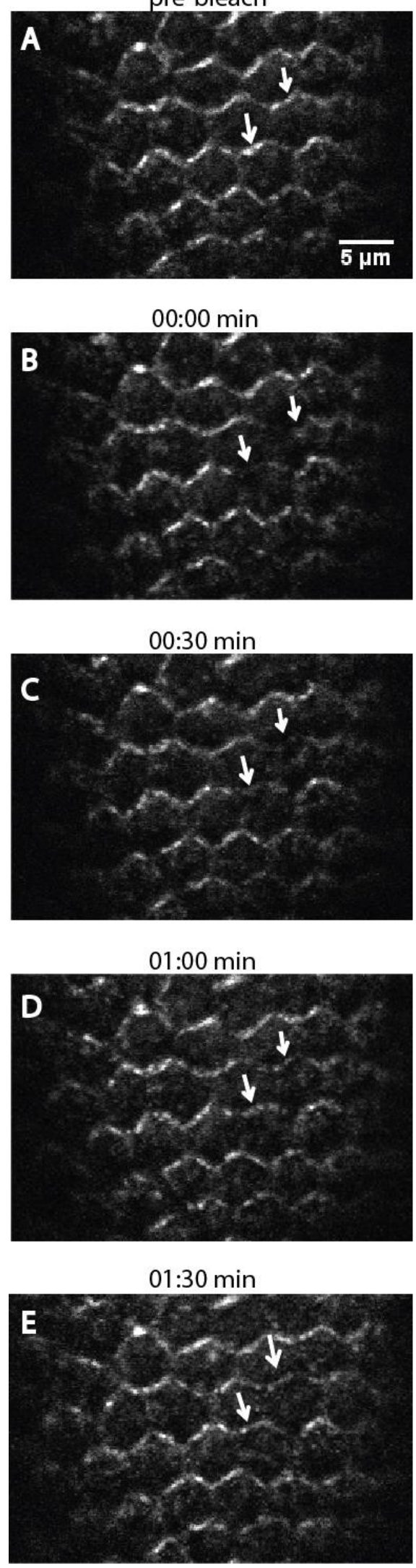

thorax cell
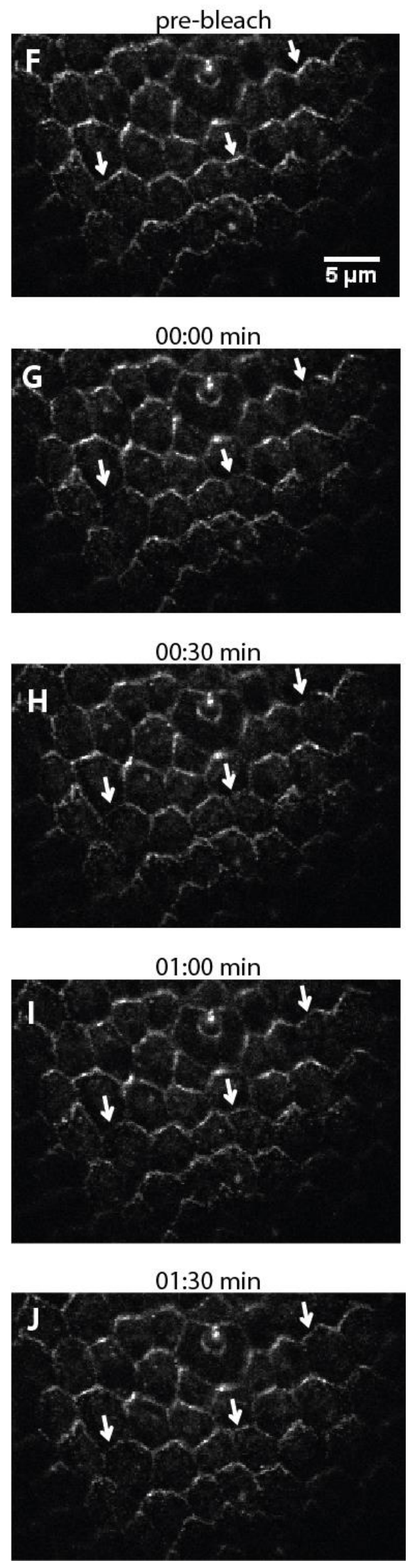
Table 1. PPE genes transgenic constructs with different fluorescent tags were inserted into various landing sites in Drosophila genome, attp1, attp2 or vk00027. Each stock rescued corresponding PPE gene mutant. There were only two stocks that showed zig-zag accumulation of the PPE proteins, and they are marked by blue in the table. In the other transgenic lines enough protein was produced to provide rescue but not enough to see by confocal microscopy. 
Table 1 Landing sites test for in, fy and frtz transgenic proteins expression

\begin{tabular}{|c|c|c|c|}
\hline \multirow{2}{*}{ Genotype } & Landing site & Rescue or not & Zig-zag expression \\
\hline \multirow{2}{*}{ pWUM6-in-venus } & attp1 & Yes & No \\
\cline { 2 - 4 } & vk00027 & Yes & No \\
\cline { 2 - 4 } & attp 2 & Yes & No \\
\hline \multirow{2}{*}{ pWUM6-venus-in } & attp1 & Yes & Yes \\
\hline \multirow{2}{*}{$p$ WUM6-fy-mcerulean3 } & attp1 & Yes & No \\
\cline { 2 - 4 } & vk00027 & Yes & No \\
\hline \multirow{2}{*}{$p W U M 6-f r t z-m c h e r r y$} & attp1 & Yes & Yes \\
\cline { 2 - 4 } & vk00027 & Yes & No \\
\hline
\end{tabular}




\section{Reference}

Adler, P.N., Charlton, J., and Liu, J. (1998). Mutations in the cadherin superfamily member gene dachsous cause a tissue polarity phenotype by altering frizzled signaling. Development $125,959-968$.

Adler, P.N., Zhu, C., and Stone, D. (2004). Inturned localizes to the proximal side of wing cells under the instruction of upstream planar polarity proteins. Curr Biol 14, 2046-2051.

Boch, J. (2011). TALEs of genome targeting. Nat Biotechnol 29, 135-136.

Boch, J., Scholze, H., Schornack, S., Landgraf, A., Hahn, S., Kay, S., Lahaye, T., Nickstadt, A., and Bonas, U. (2009). Breaking the code of DNA binding specificity of TAL-type III effectors. Science 326, 1509-1512.

Chae, J., Kim, M.J., Goo, J.H., Collier, S., Gubb, D., Charlton, J., Adler, P.N., and Park, W.J. (1999). The Drosophila tissue polarity gene starry night encodes a member of the protocadherin family. Development 126, 5421-5429.

Collier, S., and Gubb, D. (1997). Drosophila tissue polarity requires the cell-autonomous activity of the fuzzy gene, which encodes a novel transmembrane protein. Development 124 , 4029-4037. 
Collier, S., Lee, H., Burgess, R., and Adler, P. (2005). The WD40 repeat protein fritz links cytoskeletal planar polarity to frizzled subcellular localization in the Drosophila epidermis. Genetics 169, 2035-2045.

Cong, L., Ran, F.A., Cox, D., Lin, S., Barretto, R., Habib, N., Hsu, P.D., Wu, X., Jiang, W., Marraffini, L.A., et al. (2013). Multiplex genome engineering using CRISPR/Cas systems. Science 339, 819-823.

Curtin, J.A., Quint, E., Tsipouri, V., Arkell, R.M., Cattanach, B., Copp, A.J., Henderson, D.J., Spurr, N., Stanier, P., Fisher, E.M., et al. (2003). Mutation of Celsr1 disrupts planar polarity of inner ear hair cells and causes severe neural tube defects in the mouse. Curr Biol 13, 1129-1133.

Durai, S., Mani, M., Kandavelou, K., Wu, J., Porteus, M.H., and Chandrasegaran, S. (2005). Zinc finger nucleases: custom-designed molecular scissors for genome engineering of plant and mammalian cells. Nucleic Acids Res 33, 5978-5990.

Epinat, J.C., Arnould, S., Chames, P., Rochaix, P., Desfontaines, D., Puzin, C., Patin, A., Zanghellini, A., Paques, F., and Lacroix, E. (2003). A novel engineered meganuclease induces homologous recombination in yeast and mammalian cells. Nucleic Acids Res 31, 2952-2962.

Feiguin, F., Hannus, M., Mlodzik, M., and Eaton, S. (2001). The ankyrin repeat protein Diego mediates Frizzled-dependent planar polarization. Dev Cell 1, 93-101. 
Fischer, E., Legue, E., Doyen, A., Nato, F., Nicolas, J.F., Torres, V., Yaniv, M., and Pontoglio, M. (2006). Defective planar cell polarity in polycystic kidney disease. Nat Genet 38, 21-23.

Gratz, S.J., Cummings, A.M., Nguyen, J.N., Hamm, D.C., Donohue, L.K., Harrison, M.M., Wildonger, J., and O'Connor-Giles, K.M. (2013a). Genome engineering of Drosophila with the CRISPR RNA-guided Cas9 nuclease. Genetics 194, 1029-1035.

Gratz, S.J., Wildonger, J., Harrison, M.M., and O'Connor-Giles, K.M. (2013b). CRISPR/Cas9mediated genome engineering and the promise of designer flies on demand. Fly (Austin) 7, 249255.

Gubb, D., and Garcia-Bellido, A. (1982). A genetic analysis of the determination of cuticular polarity during development in Drosophila melanogaster. J Embryol Exp Morphol 68, 37-57.

Hamblet, N.S., Lijam, N., Ruiz-Lozano, P., Wang, J., Yang, Y., Luo, Z., Mei, L., Chien, K.R., Sussman, D.J., and Wynshaw-Boris, A. (2002). Dishevelled 2 is essential for cardiac outflow tract development, somite segmentation and neural tube closure. Development 129, 5827-5838.

He, B., and Adler, P.N. (2002). The frizzled pathway regulates the development of arista laterals. BMC developmental biology 2, 7. 
Henderson, D.J., Conway, S.J., Greene, N.D., Gerrelli, D., Murdoch, J.N., Anderson, R.H., and Copp, A.J. (2001). Cardiovascular defects associated with abnormalities in midline development in the Loop-tail mouse mutant. Circ Res 89, 6-12.

Henderson, D.J., Phillips, H.M., and Chaudhry, B. (2006). Vang-like 2 and noncanonical Wnt signaling in outflow tract development. Trends Cardiovasc Med 16, 38-45.

Kandavelou, K., and Chandrasegaran, S. (2009). Custom-designed molecular scissors for sitespecific manipulation of the plant and mammalian genomes. Methods in molecular biology 544, 617-636.

Klingensmith, J., Nusse, R., and Perrimon, N. (1994). The Drosophila segment polarity gene dishevelled encodes a novel protein required for response to the wingless signal. Genes Dev 8 , 118-130.

Ran, F.A., Hsu, P.D., Wright, J., Agarwala, V., Scott, D.A., and Zhang, F. (2013). Genome engineering using the CRISPR-Cas9 system. Nat Protoc 8, 2281-2308.

Sekelsky, J.J., Newfeld, S.J., Raftery, L.A., Chartoff, E.H., and Gelbart, W.M. (1995). Genetic characterization and cloning of mothers against dpp, a gene required for decapentaplegic function in Drosophila melanogaster. Genetics 139, 1347-1358. 
Strutt, D., and Warrington, S.J. (2008). Planar polarity genes in the Drosophila wing regulate the localisation of the FH3-domain protein Multiple Wing Hairs to control the site of hair production. Development 135, 3103-3111.

Strutt, H., Warrington, S.J., and Strutt, D. (2011). Dynamics of core planar polarity protein turnover and stable assembly into discrete membrane subdomains. Dev Cell 20, 511-525.

Taylor, J., Abramova, N., Charlton, J., and Adler, P.N. (1998). Van Gogh: a new Drosophila tissue polarity gene. Genetics 150, 199-210.

Wang, Y., Yan, J., Lee, H., Lu, Q., and Adler, P.N. (2014). The proteins encoded by the Drosophila Planar Polarity Effector genes inturned, fuzzy and fritz interact physically and can re-pattern the accumulation of "upstream" Planar Cell Polarity proteins. Dev Biol 394, 156-169.

Wong, L.L., and Adler, P.N. (1993). Tissue polarity genes of Drosophila regulate the subcellular location for prehair initiation in pupal wing cells. J Cell Biol 123, 209-221.

Yang, C.H., Axelrod, J.D., and Simon, M.A. (2002). Regulation of Frizzled by fat-like cadherins during planar polarity signaling in the Drosophila compound eye. Cell 108, 675-688.

Zeidler, M.P., Perrimon, N., and Strutt, D.I. (1999). The four-jointed gene is required in the Drosophila eye for ommatidial polarity specification. Curr Biol 9, 1363-1372. 


\section{Chapter 5}

\section{General discussion}

The goal of this dissertation is to get a better understanding of the mechanisms by which downstream components inturned (in), fuzzy (fy) and fritz (fritz) in the frizzled (fz)/starry night (stan) signaling pathway regulate planar cell polarity in Drosophila. In chapter 2, I showed that PPE proteins interact physically and form a protein complex in the wing cells and that the accumulation of one protein can modulate the accumulation of another in wing cells. More interesting, our research suggest the epistasis structure of the $f z /$ stan signaling pathway is context dependent. When over expressed the PPE proteins can alter both the subcellular location and level of accumulation of the upstream proteins. In chapter 3, I described a true in null mutant - a precise deletion $\left(i n^{P D}\right)$ made using the CRISPR/Cas9 genome editing technique. Using in ${ }^{P D}$, we found frtz has both in dependent and independent functions. I also presented the direct interaction between PPE protein Fy/Frtz and PCP core protein Dsh. The interaction between Frtz and Dsh comes from multiples locations in Frtz protein. In chapter 4, I focused on

the dynamic property of the Frtz protein using a modified $f r t z$ gene $-f_{r t z}{ }^{\text {meonGreen }}$, which was generated using the CRISPR/Cas9 genome editing technique. Our data suggest the frtz $^{\text {mNeonGreen }}$ fly is good for studying the Frtz protein activity in various tissues and structures. Time-lapse imaging data shows Frtz protein is preferentially distributed to discrete membrane subdomains ("puncta"), and the puncta are very dynamic at the subcellular level.

\section{Multiple roles of $\mathrm{frtz}$ in regulating planar cell polarity}

The microtubule and actin skeleton are involved in wing hair development. Disruption of actin or microtubules with drugs results in clustered hairs with relatively normal polarity 
(Geng et al., 2000; Turner and Adler, 1998). Our previous data shows when over expressed Frtz results in a dramatic delay in hair outgrowth and abnormal bristle morphology, which is not shared by other members of the group, suggesting the possibility that at least when over expressed Frtz could inhibit the activation of the actin cytoskeleton (Wang et al., 2014). Also, WDPCP, mammalian homolog of Drosohila Frtz has been found to regulate the actin cytoskeleton in mouse. The organization of actin filaments and focal contacts was markedly changed in wdpcp-deficient cells (Cui et al., 2013). Together, it suggests a role of Drosophila frtz in regulating actin cytoskeleton. Using our newly generated $f r t z^{\text {mNeonGreen }}$ fly, we will be able to demonstrate if Frtz accumulates in hairs and bristles and study if Frtz protein and actin bundles co-localize there. If we observe the co-localization between Frtz protein and actin bundles in bristles or hairs, in vitro actin assays can be done to test the ability of purified Frtz protein to regulate actin polymerization or bundling. However, purifying the protein can be time and labor consuming and the purified protein may not be active in vitro. Alternatively, a relatively easier approach can be done by studying the Frtz protein accumulation in mutants that alter the distribution of F-actin in hairs or bristles. If there is a direct relationship between actin and Frtz we should see a change in Frtz distribution. Seeing such a result would indicate the more laborious in vitro biochemical approach is warrented.

Besides a potential role in regulating the actin cytoskeleton, Frtz may also have other functions in regulating planar cell polarity which is independent of the $f z /$ stan pathway. Our experiments show mild overexpression of Frtz partially rescued $i n^{P D}$ mutant indicating that Frtz has In independent function. Consistent with this hypothesis is that the gain of function phenotype associated with the high level over expression of Frtz was not blocked in an in mutant (Wang, 2014). Nor was it completely blocked in $f z$ or $d s h$ mutants. It is possible that 
Frtz might regulate the actin cytoskeleton (Cui et al., 2013; Kim et al., 2010) independently of its role in the $f z /$ stan pathway. It is also possible that Frtz may function as an effector in another PCP pathway, such as the dachsous(ds)/fat(ft) pathway. Although several group reported that $d s / f t$ pathway functions upstream of $f z /$ stan pathway, such as in Drosophila compound eye (Yang et al., 2002), in other tissues that is not the case. It is clear that the $d s / f t$ and $f z / s t a n$ pathways function in parallel in the abdomen (Casal et al., 2006). Double mutants of one gene in $d s / f t$ pathway and one gene in $f z /$ stan pathway showed a more severe phenotype than any single mutant. We could test if $f r t z$ is the effector of the $d s / f t$ pathway using our newly generated $\mathrm{frtz}^{\mathrm{mNeonGreen}}$ fly, with which we were able to detect the asymmetric accumulation of Frtz in various tissues. One approach to test this hypothesis is studying the Frtz accumulation in a $f t$ or $d s$ mutant background to see if there is a change in the Frtz accumulation pattern.

\section{The interaction between PPE proteins and PCP core proteins}

Previous data shows In, Fy and Frtz proteins accumulate on proximal side of wing cells and function together in a protein complex (Adler et al., 2004; Collier et al., 2005; Strutt and Warrington, 2008; Wang et al., 2014). However, how PPE proteins or the PPE protein complex are recruited to the proximal edge of the cell is still unclear. Our hypothesis is that the upstream PCP core proteins recruit the downstream components In, Fy and Frtz protein/ protein complex to the proximal edge of wing cells. The recruiting force could be positive and come from proximal localized protein, such as Vang or Pk, or negative and come from the distally localized proteins Dsh, Dgo or Fz. We recently obtained clear evidence that Fy and Frtz interact directly with Dsh using both yeast-two-hybrid and co-immunoprecipitation assays. We also tested the interaction between Pk and PPE proteins In, Fy and Frtz using the yeast-two-hybrid system respectively and we didn't observe the positive interactions. Dsh asymmetrically accumulates 
on the distal side of wing cell which is opposite to the side where Frtz and Fy expressed, whereas $\mathrm{Pk}$ accumulates proximally where PPE proteins are expressed. Our data suggest the antagonistic interaction between Dsh and PPE proteins results in the asymmetric accumulation of PPE proteins in wing cells. However, the interaction between proximally accumulated proteins Vang/Pk and PPE proteins remains possible. The interaction may not be direct, requiring the presence of other factors. It is also possible that Vang or a combination of Pk and Vang functions to recruit the PPE proteins. One approach to test this hypothesis is coimmunoprecipitation of these proteins in living cells.

The antagonistic force between a proximal localized protein and a distally localized protein has been demonstrated before and it was shown important for regulating planar cell polarity in fly. For example, interaction between $\mathrm{Pk}$ and $\mathrm{Dsh}$ could prevent Dsh from accumulating on proximal side and $\mathrm{Pk}$ from accumulating on distal side (Bastock et al., 2003; Tree et al., 2002). The negative interaction may come from the proteasome-mediated degradation (Adler, 2012; Lecker et al., 2006; Narimatsu et al., 2009). Smurfs are ubiquitin ligases that regulate signaling, cell polarity and motility through spatiotemporally restricted ubiquitination of diverse substrates (Izzi and Attisano, 2006). It was reported before that Smurfs control PCP and convergence and extension movements in mouse. Ubiquitin dependent Pk degradation by Smurfs has been implicated in regulating PCP (Narimatsu et al., 2009). The antagonistic force between Fy/ Frtz and Dsh may also come from the ubiquitin-proteasome mediated degradation. In mouse Smurf1 and Smurf2 were found form a protein complex with phosphorylated Dvl2 (mammalian Dsh homolog) that targets Pk for degradation (Narimatsu et al., 2009). The Smurfs and Dsh protein complex may also target PPE proteins resulting in the asymmetric accumulation on proximal edge of wing cells. 
Dynamic properties of the PPE proteins

The time-lapse imaging of both ubiquitin-myc-frtz-GFP and frtz ${ }^{\text {mNeonGreen }}$ flies demonstrate that Frtz protein is very dynamic in various tissues. The Frtz protein particles are moving in the cytoplasm or along the cell boundary all the time. Even the cell boundary associated Frtz protein can go back to cytoplasm again. The FRAP analysis also suggest Frtz protein is very dynamic. A large amount of fluorescence recovered quickly after photobleaching. To understand how Frtz protein is recruited asymmetrically to the proximal edge of the wing cell, one can study the Frtz protein moving pattern. Live imaging of fluorescently tagged PCP core proteins in pupal wings showed that Fz- and Dsh-containing particles travel across the cell in a proximal-to-distal direction, and the directed PCP transport is mediated by an array of subapical, noncentrosomal microtubules that align along the proximal-distal axis (Harumoto et al., 2010; Matis et al., 2014; Shimada et al., 2006). We can analyze if Frtz containing protein particle has similar moving pattern in cells to understand how it is recruited.

Although there is no data showing if the movement of In and Frtz is correlated, that seems likely to be the case. Using a modified $i^{m R u b y 2}$ fly, which I am currently trying to make we will be able to test his possibility. All PPE proteins are recruited asymmetrically to the proximal edge of wing cells and form a protein complex there (Adler et al., 2004; Collier et al., 2005; Strutt and Warrington, 2008; Wang et al., 2014). However, we don't know if the protein complex is formed before or after recruiting proximally. By expressing $i n^{m R u b y 2}$ and $f r t z^{m \text { NeonGreen }}$ in the same cells and studying their movement we should be able to determine this. 


\section{Reference}

Adler, P.N. (2012). The frizzled/stan pathway and planar cell polarity in the Drosophila wing. Curr Top Dev Biol 101, 1-31.

Adler, P.N., Zhu, C., and Stone, D. (2004). Inturned localizes to the proximal side of wing cells under the instruction of upstream planar polarity proteins. Curr Biol 14, 2046-2051.

Bastock, R., Strutt, H., and Strutt, D. (2003). Strabismus is asymmetrically localised and binds to Prickle and Dishevelled during Drosophila planar polarity patterning. Development 130, 3007-3014.

Casal, J., Lawrence, P.A., and Struhl, G. (2006). Two separate molecular systems, Dachsous/Fat and Starry night/Frizzled, act independently to confer planar cell polarity. Development 133, 4561-4572.

Collier, S., Lee, H., Burgess, R., and Adler, P. (2005). The WD40 repeat protein fritz links cytoskeletal planar polarity to frizzled subcellular localization in the Drosophila epidermis. Genetics 169, 2035-2045.

Cui, C., Chatterjee, B., Lozito, T.P., Zhang, Z., Francis, R.J., Yagi, H., Swanhart, L.M., Sanker, S., Francis, D., Yu, Q., et al. (2013). Wdpcp, a PCP protein required for ciliogenesis, regulates directional cell migration and cell polarity by direct modulation of the actin cytoskeleton. PLoS Biol 11, e1001720. 
Geng, W., He, B., Wang, M., and Adler, P.N. (2000). The tricornered gene, which is required for the integrity of epidermal cell extensions, encodes the Drosophila nuclear DBF2-related kinase. Genetics 156, 1817-1828.

Harumoto, T., Ito, M., Shimada, Y., Kobayashi, T.J., Ueda, H.R., Lu, B., and Uemura, T. (2010). Atypical cadherins Dachsous and Fat control dynamics of noncentrosomal microtubules in planar cell polarity. Dev Cell 19, 389-401.

Izzi, L., and Attisano, L. (2006). Ubiquitin-dependent regulation of TGFbeta signaling in cancer. Neoplasia 8, 677-688.

Kim, S.K., Shindo, A., Park, T.J., Oh, E.C., Ghosh, S., Gray, R.S., Lewis, R.A., Johnson, C.A., Attie-Bittach, T., Katsanis, N., et al. (2010). Planar cell polarity acts through septins to control collective cell movement and ciliogenesis. Science 329, 1337-1340.

Lecker, S.H., Goldberg, A.L., and Mitch, W.E. (2006). Protein degradation by the ubiquitinproteasome pathway in normal and disease states. J Am Soc Nephrol 17, 1807-1819.

Matis, M., Russler-Germain, D.A., Hu, Q., Tomlin, C.J., and Axelrod, J.D. (2014). Microtubules provide directional information for core PCP function. eLife 3, e02893. 
Narimatsu, M., Bose, R., Pye, M., Zhang, L., Miller, B., Ching, P., Sakuma, R., Luga, V.,

Roncari, L., Attisano, L., et al. (2009). Regulation of planar cell polarity by Smurf ubiquitin ligases. Cell 137, 295-307.

Shimada, Y., Yonemura, S., Ohkura, H., Strutt, D., and Uemura, T. (2006). Polarized transport of Frizzled along the planar microtubule arrays in Drosophila wing epithelium. Dev Cell 10, 209-222.

Strutt, D., and Warrington, S.J. (2008). Planar polarity genes in the Drosophila wing regulate the localisation of the FH3-domain protein Multiple Wing Hairs to control the site of hair production. Development 135, 3103-3111.

Tree, D.R., Shulman, J.M., Rousset, R., Scott, M.P., Gubb, D., and Axelrod, J.D. (2002). Prickle mediates feedback amplification to generate asymmetric planar cell polarity signaling. Cell 109, 371-381.

Turner, C.M., and Adler, P.N. (1998). Distinct roles for the actin and microtubule cytoskeletons in the morphogenesis of epidermal hairs during wing development in Drosophila. Mech Dev 70, 181-192.

Wang, Y., Yan, J., Lee, H., Lu, Q., and Adler, P.N. (2014). The proteins encoded by the Drosophila Planar Polarity Effector genes inturned, fuzzy and fritz interact physically and can re-pattern the accumulation of "upstream" Planar Cell Polarity proteins. Dev Biol 394, 156-169. 
Yang, C.H., Axelrod, J.D., and Simon, M.A. (2002). Regulation of Frizzled by fat-like cadherins during planar polarity signaling in the Drosophila compound eye. Cell 108, 675-688. 